\title{
A Two-Dimensional Riemann Solver with Self-Similar Sub-Structure - Alternative Formulation Based on Least Squares Projection
}

By

\author{
Dinshaw S. Balsara ${ }^{1}$ (dbalsara@nd.edu), Jeaniffer Vides ${ }^{2}$ (jeaniffer.vides@cea.fr), Katharine \\ Gurski $^{3}$ (kgurski@howard.edu), Boniface Nkonga² (boniface.nkonga@unice.fr), Michael \\ Dumbser $^{4}$ (michael.dumbser@ing.unitn.it), Sudip Garain ${ }^{1}$ (sgarain@nd.edu) \& Edouard Audit ${ }^{5}$ \\ (edouard.audit@cea.fr) \\ ${ }^{1}$ Physics Department, University of Notre Dame, USA \\ ${ }^{2}$ Universiteé de Nice-Sophia Antipolis, UMR CNRS \& Inria Sophia Antipolis, France \\ ${ }^{3}$ Applied Mathematics Department, Howard University, USA \\ ${ }^{4}$ Laboratory of Applied Mathematics, Department of Civil, Environmental and \\ Mechanical Engineering, University of Trento, Italy \\ ${ }^{5}$ Maison de la Simulation, CEA-CNRS-Inria-UPSud, France
}

\begin{abstract}
Just as the quality of a one-dimensional approximate Riemann solver is improved by the inclusion of internal sub-structure, the quality of a multidimensional Riemann solver is also similarly improved. Such multidimensional Riemann problems arise when multiple states come together at the vertex of a mesh. The interaction of the resulting one-dimensional Riemann problems gives rise to a strongly-interacting state. We wish to endow this strongly-interacting state with physically-motivated sub-structure. The self-similar formulation of Balsara [16] proves especially useful for this purpose. While that work is based on a Galerkin projection, in this paper we present an analogous self-similar formulation that is based on a different interpretation. In the present formulation, we interpret the shock jumps at the boundary of the strongly-interacting state quite literally. The enforcement of the shock jump conditions is done with a least squares projection (Vides, Nkonga \& Audit [67]). With that interpretation, we again show that the multidimensional Riemann solver can be endowed with sub-structure. However, we find that the most efficient implementation arises when we use a flux vector splitting and a least squares projection. An alternative formulation that is based on the full characteristic matrices is also presented. The multidimensional Riemann solvers that are demonstrated here use one-dimensional HLLC Riemann solvers as building blocks.
\end{abstract}

Several stringent test problems drawn from hydrodynamics and MHD are presented to show that the method works. Results from structured and unstructured meshes demonstrate the 
versatility of our method. The reader is also invited to watch a video introduction to multidimensional Riemann solvers on http://www.nd.edu/ dbalsara/Numerical-PDE-Course. 


\section{I) Introduction}

Riemann solvers play an important role in the numerical solution of hyperbolic systems of conservation laws. The one-dimensional Riemann problem is a self-similar solution that results from a discontinuity between two constant states. Multidimensional Riemann solvers have also been designed and we focus on a certain class of multidimensional Riemann solvers here (Wendroff [68], Balsara [4], [5], [16], Balsara, Dumbser \& Abgrall [15], Vides, Nkonga \& Audit[68], Balsara \& Dumbser [17]). Such Riemann solvers are applied at the vertices of a twodimensional mesh. Many states come together at a vertex from different directions, making it possible to communicate the multidimensionality of the flow to the multidimensional Riemann solver. At the vertex, the job of the multidimensional Riemann solver is to approximate the selfsimilar multidimensional structure that emanates from the vertex. While self-similarity has not been used much in the design of one-dimensional Riemann solvers, it is crucially important in the development of multidimensional Riemann solvers (Balsara [16], Balsara \& Dumbser [17]). This has prompted the name of MuSIC Riemann solvers, where MuSIC stands for "Multidimensional, Self-similar, strongly-Interacting, Consistent". Such Riemann solvers are multidimensional; they draw on the self-similarity of the problem; they focus on the stronglyinteracting state that results when multiple one-dimensional Riemann solvers interact; and the design relies on establishing consistency with the conservation law. MuSIC Riemann solvers that rely on a Galerkin projection to obtain the self-similar variation in the strongly interacting state have been presented (Balsara [16], Balsara \& Dumbser [17]). An alternative projection method consists of least squares and Vides, Nkonga \& Audit[68] developed a multidimensional Riemann solver without sub-structure based on such a projection. The goal of this paper is to show that least squares projection can also be used to design a MuSIC Riemann that retains sub-structure.

Several excellent one-dimensional Riemann solvers have been designed. There are exact Riemann solvers from Godunov [41],[42] and van Leer [66] and two-shock approximations thereof (Colella [27], Colella \& Woodward [29]). See also the work of Chorin [25]. The linearized Riemann solver by Roe [52] and the HLL/HLLE/HLLEM Riemann solvers (Harten, Lax \& van Leer [44], Einfeldt [34], Einfeldt et al. [35]) and the local Lax-Friedrichs (LLF) Riemann solver (Rusanov [56]) have also seen frequent use. Toro, Spruce and Speares [62] [63], [64], Chakraborty \& Toro [24] and Batten et al. [20] produced an HLLC class of Riemann solvers which have become very popular. See also, Billett \& Toro [21]. Osher and Solomon [51] and Dumbser \& Toro [33] presented approximate Riemann solvers based on path integral methods in phase space. In Balsara [16] we showed that the principle of self-similarity can be used to advantage with the result that any of the above-mentioned one-dimensional Riemann solvers can be used as a building block in the design of multidimensional Riemann solvers by relying on a Galerkin projection. The present paper continues this line of inquiry by showing that a least squares projection can also be used. The results are instantiated for the very popular HLLC class of Riemann solvers. 
Magnetohydrodynamics (MHD) is an interesting example of a hyperbolic system with a more complex wave foliation. One-dimensional linearized Riemann solvers for numerical MHD have been designed (Roe \& Balsara [54], Cargo and Gallice [23], Balsara [6]). HLLC Riemann solvers, capable of capturing mesh-aligned contact discontinuities, have been presented by Gurski [43] and Li [47]. Miyoshi and Kusano [49] drew on Gurski's work to design an HLLD Riemann solver for MHD. It is, therefore, interesting to show that MHD can also be accommodated within our formulation. MHD is a system with an involution constraint, where the divergence of the magnetic field is always zero. Balsara \& Spicer [7] showed that this is assured within the context of a higher order Godunov scheme by using the upwinded fluxes at the edges of the mesh to update the magnetic fields that are collocated at the faces of a mesh. Gardiner \& Stone [38], [39] have claimed that the dissipation in those upwinded fluxes needs to be doubled all the time in order to stabilize the method. A substantial body of work now exists to show that the suggestion of Gardiner \& Stone is completely unnecessary when multidimensional Riemann solvers are used to provide a properly upwinded electric field at the edges of the mesh (Balsara [5], Vides, Nkonga \& Audit[67], Balsara \& Dumbser [18]). Indiscriminate doubling of the dissipation, as per Gardiner \& Stone's suggestion, can indeed lead to excessive dissipation of the magnetic field in the direction that is transverse to the upwind direction. The present paper reinforces that finding.

For the sake of completeness, and also for the sake of putting this work in context, we mention that there have been prior effort at designing multidimensional Riemann solvers. One strain of research consists of trying to build some level of multidimensionality into one dimensional Riemann solvers (Colella [28], Saltzman [57], LeVeque [46]). Another line of early effort tried to incorporate genuine multidimensionality and did not meet with much initial success (Roe [53], Rumsey, van Leer \& Roe [55]). Abgrall [1], [2] made a big breakthrough by formulating a genuinely multidimensional Riemann solver for CFD that worked. Further advances were also reported (Fey [36], [37], Gilquin, Laurens \& Rosier [40], Brio, Zakharian \& Webb [22], Lukacsova-Medvidova et al. [48]). Most of these above-mentioned genuinely multidimensional Riemann solvers did not see much use because they were difficult to implement. Wendroff [68] formulated a two-dimensional HLL Riemann solver, but his method was also not easy to implement. A video introduction to multidimensional Riemann solvers is available on the following website: http://www.nd.edu/ dbalsara/Numerical-PDE-Course .

Balsara [4] devised a two-dimensional HLL Riemann solver with simple closed form expressions for the fluxes that were easy to implement. In Balsara [5] it was shown that one can impart sub-structure to the HLL state, yielding a multidimensional HLLC Riemann solver. Balsara, Dumbser \& Abgrall [15] extended this formulation to accommodate unstructured meshes. The previous three papers formulated the multidimensional Riemann problem by integrating the conservation law over the extent of the wave model in space-time. In their study of the multidimensional Riemann problem, Schulz-Rinne, Collins \& Glaz [58] had shown that the one-dimensional Riemann problems interact amongst themselves to form a self-similarly 
evolving strongly-interacting state. This strongly-interacting state emerges by propagating into the one-dimensional Riemann problems via an evolving boundary. We refer to this boundary as the boundary of the multidimensional wave model because it contains the strongly-interacting state. The wave models in all the multidimensional Riemann solvers incorporate this selfsimilarity. But there is a deeper way in which self-similarity can be used, as shown in the next paragraph.

The self-similarly evolving strongly-interacting state is an inevitable consequence of wavefronts propagating into the one-dimensional Riemann problems. Seizing on this insight, Balsara [16] presented a self-similar formulation of the multidimensional Riemann problem. Balsara \& Dumbser [17] extended these ideas to unstructured meshes. In the first of those two papers, a Galerkin projection method was devised which had the pleasant consequence of deriving most of its information about the sub-structure in the strongly interacting state via boundary integrals applied to the self-similarly expanding multidimensional wave model. In that fashion, the Galerkin projection method picks up on the physical idea that Lagrangian fluxes carry mass, momentum and energy through the moving boundary of the multidimensional wave model. That is, the mathematical formulation reproduces the physics of the problem. The correspondence between the space-time formulation of the multidimensional Riemann problem and the analogous formulation in similarity variables has also been shown in Balsara [16].

An alternative viewpoint was presented by Vides, Nkonga \& Audit [68] with a least squares projection method that also required the balancing of Lagrangian fluxes across the moving boundary of the multidimensional wave model. This can be viewed as a way to enforce shock jumps across the boundaries of the wave model. (Because of the least squares procedure, the enforcement of shock jumps is never quite exact. Instead it should be viewed as an approximate imposition of shock jumps. However, the integration that takes place in a Galerkin projection can also be viewed as an approximation process.) The resulting multidimensional Riemann solver by Vides, Nkonga \& Audit [68] was an HLL-type Riemann solver and did not retain sub-structure. In this paper we show how the least squares projection can also be used to endow substructure to the multidimensional Riemann problem. Thus by having two complementary viewpoints for designing MuSIC Riemann solvers with sub-structure, via Galerkin projection and via least squares projection, we have a better perspective on the design of multidimensional Riemann solvers.

Section II sets up the problem and provides details associated with the construction of the multidimensional wave model. Section III provides details about the least squares projection and how it works within the context of a self-similar formulation where shock jumps are explicitly enforced at the boundaries of the wave model. Section IV presents accuracy analysis and Section $\mathrm{V}$ presents several stringent test problems. Section VI presents our conclusions. Proofs of the least squares projection have been catalogued in Appendices A and B.

\section{II) Problem Setup and Construction of the Multidimensional Wave Model}


Consider a hyperbolic conservation law in two dimensions, $\partial_{t} \mathbf{U}+\partial_{x} \mathbf{F}+\partial_{y} \mathbf{G}=0$, where $\mathbf{U}$ is an $N$-component vector of conserved variables and $\mathbf{F}$ and $\mathbf{G}$ are the fluxes in the $x$ - and $y$ directions. At any one-dimensional boundary between zones, a one-dimensional Riemann problem is likely to arise. Fig. 1 shows such a one-dimensional boundary along with a onedimensional Riemann problem that develops between states $\mathbf{U}_{1}$ and $\mathbf{U}_{2}$. At any vertex of the mesh, where the multiple zones come together, the one-dimensional Riemann problems will interact strongly amongst themselves. Fig. 1 displays such a vertex where five states, $\mathbf{U}_{1}$ to $\mathbf{U}_{5}$, come together at the vertex $\mathrm{O}$. We do not show the other one-dimensional Riemann problems in Fig. 1 just to keep it uncluttered. However, Fig. 1 does establish the fact that there is a unit vector $\mathbf{n}_{1} \equiv n_{1 x} \mathbf{x}+n_{1 y} \mathbf{y}$ that runs parallel to the interface between states $\mathbf{U}_{1}$ and $\mathbf{U}_{2}$. The unit vector that runs perpendicular to $\mathbf{n}_{1}$, and does so in the counter-clockwise direction, is denoted by $\mathbf{t}_{1} \equiv-n_{1 y} \mathbf{x}+n_{1 x} \mathbf{y}$. The extremal speeds of the one-dimensional Riemann problem in the $\mathbf{t}_{1}$ direction are then denoted by $S_{\mathbf{t} 1}^{-}$and $S_{\mathbf{t} 1}^{+}$. The thick dotted line in Fig. 1 is intended to show a contact discontinuity that develops in a one-dimensional HLLC Riemann solver. The contact discontinuity separates two post-shock states which we denote as $\mathbf{U}_{1}^{*_{-}}$and $\mathbf{U}_{1}^{*+}$. Associated with the state $\mathbf{U}_{1}^{*-}$ we have the fluxes $\mathbf{F}_{1}^{{ }^{*-}}$ and $\mathbf{G}_{1}^{*-}$. Similarly, associated with the state $\mathbf{U}_{1}^{*_{+}}$we also have the fluxes $\mathbf{F}_{1}^{*+}$ and $\mathbf{G}_{1}^{*_{+}}$. While Fig. 1 only shows five incoming states at the vertex of a mesh, the notation extends naturally to any number of states. Thus our method generally allows for a set of states $\left\{\mathbf{U}_{i}: i=1, \ldots, I_{\max }\right\}$ coming together at a vertex. The natural ordering of the states is taken to be counterclockwise. These states are cyclically numbered so that $\mathbf{U}_{i+1}$ is identical to $\mathbf{U}_{1}$ when $i=I_{\max }$. For the sake of simplicity, we substantiate the method by showing the schematic diagram of an HLLC Riemann solver in Fig. 1, but any one-dimensional Riemann solver can be used.

When the one-dimensional Riemann problems interact with each other at the vertex $\mathrm{O}$ in Fig. 1, the interaction produces a strongly-interacting state (Schulz-Rinne et al. [58]). By this point in time, several authors (Wendroff [68], Balsara [4], [5], [16], Balsara, Dumbser \& Abgrall [15], Vides, Nkonga \& Audit[67], Balsara \& Dumbser [17]) have understood that the region of strong interaction holds the key to the design of a multidimensional Riemann solver. Conceptually, it helps to identify the extent of the region of strong interaction as much as possible. Thus we say that the region of strong interaction is bounded by the multidimensional wave model, in quite the same way that a one-dimensional Riemann problem is bounded by a one-dimensional wave model. Identification of the multidimensional wave model is usually done on the basis of entropy enforcement. In other words, the strongly interacting state should at least be large enough to contain each of the contributing one-dimensional Riemann problems. There are multiple ways of achieving this goal even on an unstructured mesh (Balsara, Dumbser \& Abgrall [15], Vides, Nkonga \& Audit[67], Balsara \& Dumbser [17]). In Fig. 2, which is drawn 
from Balsara \& Dumbser [17], we show one possible way of building the multidimensional wave model. Notice that the wave model does track the principal directions of the mesh. However, as the angular resolution of the mesh around a vertex becomes enriched, the wave model tends more closely to a Monge cone. This is how the wave model makes connection to the physics of the problem.

Focus on the pentagonal wave model shown in Fig. 2. Pictorially, it is easy to see how it has been formed from the contributing one-dimensional Riemann problems. The pentagon is defined by a specification of its vertices. Each vertex is formed by the intersection of the extremal waves emanating from adjacent zone boundaries. Focusing on the second vertex in Fig. 2 , it is formed by the interaction of the shock that emanates from the first zone boundary with speed $S_{\mathbf{t} 1}^{+}$in the $\mathbf{t}_{1}$ direction and the shock that emanates from the second zone boundary with speed $S_{\mathbf{t} 2}^{-}$in the $\mathbf{t}_{2}$ direction. It is, therefore, easy to see that the second vertex $\mathrm{P}_{2}=\left(\tilde{\xi}_{2}, \tilde{\psi}_{2}\right)$ in Fig. 2 is nothing but the intersection point of the two lines $-n_{1 y} \tilde{\xi}+n_{1 x} \tilde{\psi}=S_{\mathrm{t} 1}^{+}$and $-n_{2 y} \tilde{\xi}+n_{2 x} \tilde{\psi}=S_{\mathbf{t} 2}^{-}$. A similar exercise yields all the vertices, $\mathrm{P}_{1}$ to $\mathrm{P}_{5}$, of the pentagon shown in Fig. 2. Notice that the boundary of the multidimensional wave model is measured in units of speed because each of its vertices is formed by the intersection of two one-dimensional waves that are characterized by their speed. It is easy to see that when five states come together at a vertex the result will almost always be a convex pentagon. (Though we will discuss some exception cases shortly.) When " $I_{\max }$ " states come together at a vertex, the wave model will usually be a convex polygon with " $I_{\max }$ " sides.

Once the vertices of the pentagon are obtained, we can easily produce additional information about the wave model that is very useful for efficient computation. Fig. 2 does not only show the extremal waves of the one-dimensional Riemann problems. The dotted lines also show the contact discontinuities in the one-dimensional HLLC Riemann solvers. The intersection of each one-dimensional contact discontinuity with the associated face of the multidimensional wave model enables us to identify the points $C_{1}$ to $C_{5}$. Notice now that the segment $P_{1} C_{1}$ on the boundary of our multidimensional wave model in Fig. 2 corresponds to the constant state $\mathbf{U}_{1}^{*-}$ in Fig. 1. Likewise, the segment $\mathrm{C}_{1} \mathrm{P}_{2}$ in Fig. 2 corresponds to the constant state $\mathbf{U}_{1}^{*+}$ in Fig. 1. Since the strategy that is presented in this paper relies on a discrete imposition of shock jumps at the boundary of the multidimensional wave model, the midpoints of segments $\mathrm{P}_{1} \mathrm{C}_{1}$ and $\mathrm{C}_{1} \mathrm{P}_{2}$ also provide us with favorable locations where those shock jumps can be imposed. (It is also possible to envision a more elaborate quadrature, like Gauss-Lobatto, with more quadrature points along each of these segments; but for now we use the simplest midpoint rule.) If the one-dimensional Riemann problems have a more complicated wave foliation, those waves can also be identified by their intersection with the appropriate faces of the multidimensional wave model. In that case, 
a larger number of segments would have to be identified in each face of the multidimensional wave model.

One of the better aspects of a multidimensional Riemann solver is that the solution that it produces has to be sensitive to the underlying geometry of the mesh. As a result, in each face of the multidimensional wave model it also helps to build and store a little bit of additional geometric information. Notice, therefore, from Fig. 2 that at each face we also build a unit outward pointing normal and a unit tangent that has a counterclockwise orientation. For the face $\mathrm{P}_{1} \mathrm{P}_{2}$ in Fig. 2, for instance, those unit vectors are shown as $\boldsymbol{\eta}_{1} \equiv \eta_{1 x} \mathbf{x}+\eta_{1 y} \mathbf{y}$ and $\boldsymbol{\tau}_{1} \equiv-\eta_{1 y} \mathbf{x}+\eta_{1 x} \mathbf{y}$ respectively. These unit vectors will also be very useful in writing down the jump conditions.

When enforcing shock jump conditions at the outer boundaries of our multidimensional wave model, it is very important to know the speed with which the shock front is moving. Notice, therefore, that the construction in Figs. 1 and 2 provides the vertices of the multidimensional wave model. Recall that these vertices have units of speed. We refer to each of the flat polygonal faces in Fig. 2 as the wavefronts of the multidimensional wave model. Since we wish to enforce shock jumps later on, we identify the speeds with which these wavefronts are moving relative to the origin. Realize that this speed is measured with respect to the unit normal to the wavefront, as shown in Fig. 3. Now that we explicitly have the components of these unit normals, we can find the speeds in Fig. 3. For example, the speed $S_{1}$ in Fig. 3 is explicitly given by $S_{1}=0.5\left[\eta_{1 x}\left(\tilde{\xi}_{1}+\tilde{\xi}_{2}\right)+\eta_{1 y}\left(\tilde{\psi}_{1}+\tilde{\psi}_{2}\right)\right]$. I.e. the vector $0.5\left[\left(\tilde{\xi}_{1}+\tilde{\xi}_{2}\right) \mathbf{x}+\left(\tilde{\psi}_{1}+\tilde{\psi}_{2}\right) \mathbf{y}\right]$ corresponding to the mid-point of segment $\mathrm{P}_{1} \mathrm{P}_{2}$ is projected in the direction of the unit outward pointing normal $\boldsymbol{\eta}_{1}$ at that face.

As shown in Balsara [16], the physical variables that lie within the multidimensional wave model are most easily endowed with substructure by expanding those variables around the centroid of the wave model. Once the vertices of the multidimensional wave model are found in Figs. 2 or 3, it is possible to find the centroid of the wave model. This is shown by $\kappa$ in Fig. 4. It also helps to shift all the coordinates of the vertices of the multidimensional wave model to a frame of reference that is centered around $\kappa$. This is just a coordinate translation from $\mathrm{O}$ to $\kappa$ in Fig. 4. For more details on finding the centroid via an automated procedure, please see Balsara \& Dumbser [17]. This shifted coordinate system is also shown in Fig. 4, along with the shifted vertices of the wave model. In the shifted coordinate system we let $P_{i}=\left(\xi_{i}, \psi_{i}\right)$ be the starting coordinates of the $i^{\text {th }}$ face of our wave model and let $P_{i+1}=\left(\xi_{i+1}, \psi_{i+1}\right)$ be the ending coordinate of the same side. If the subscript " $i+1$ " exceeds the number of sides " $I_{\max }$ ", it is cyclically remapped to "1". The points $C_{i}$ are also shifted so that $C_{i}=\left(\xi_{C i}, \psi_{C i}\right)$ gives us the intersection point of the $i^{\text {th }}$ side of the multidimensional wave model with the contact discontinuity from the $i^{\text {th }}$ one-dimensional Riemann problem. The lengths $l_{i}$ for each of the sides of the wave model 
are also shown in Fig. 4. For Fig. 4, each of those lengths can also be split up into lengths that correspond to the sub-segments that constitute the wavefront. For example, for the segment $P_{1} P_{2}$ , we can also keep track of the lengths of the sub-segments $P_{1} C_{1}$ and $C_{1} P_{2}$, which we denote as $l_{P 1 C 1}$ and $l_{C 1 P 2}$. The mathematical motivation for using a shifted coordinate system will become clearer Section III.

The wave model construction described here is inspired by the original one from Wendroff [68]. However, we point out that under certain circumstances it can result in a nonconvex polygon for our wave model. This is likely to happen when the extremal speeds from the one-dimensional wave model vary greatly from one zone boundary to another. An example of such a situation is shown in Fig. 5 where the thick solid black lines correspond to the wave model that is constructed according to the previously-described construction. When such a situation arises, we revert to the wave model in Balsara [5] or Balsara, Dumbser \& Abgrall [15], which is always guaranteed to produce a convex wave model. This is shown by the thick grey lines in Fig. 5. As we will see in the next section, a convex wave model is essential for ensuring that the rest of the formulation can be defined. It is important to realize that the solid black wave model in Fig. 5 arises in situations where the flow is very non-uniform and in such situations, the larger grey-colored wave model in Fig. 5 may even be desirable because it slightly increases dissipation. When the flow is even somewhat uniform, both wave models have comparable sizes and produce comparable amounts of dissipation. If the application developer plans to develop only one multidimensional wave model, we would suggest using the one that is persistently convex.

In this section we have described the construction of the multidimensional wave model in the subsonic case. This is the case that occurs most often and is shown in Figs. 2, 3, and 4. We say that the wave model is subsonic because it overlies the origin $\mathrm{O}$ in Fig. 2. It can even turn out that for certain types of moderately supersonic flow, the multidimensional wave model is, nevertheless, subsonic. However, for certain types of strongly supersonic flows, the multidimensional wave model can become supersonic. In that case, it will not overlie the origin $O$. In that case the situation becomes very simple because the numerical fluxes that correspond to the point $\mathrm{O}$ have to be obtained from the appropriate part of the one-dimensional Riemann problem that overlies the origin $\mathrm{O}$. A constructive process for obtaining the numerical fluxes in the supersonic case is described in Balsara [16], Vides, Nkonga \& Audit [67] and Balsara \& Dumbser [17]. The last reference is especially relevant for unstructured meshes. This completes our description of the multidimensional wave model.

\section{III) Formulation of the Multidimensional Riemann Problem in Least-Squares Sense}

\section{III.a) Formulation in Similarity Variables}


The strongly interacting state evolves self-similarly, leading to a self-similar formulation (Balsara [16], Balsara \& Dumbser [17]). As shown in Fig. 2, in the subsonic case, it overlies the vertex of the mesh and will contribute to the numerical fluxes at that vertex. This is the situation that occurs most frequently in most applications and we refer to it as the subsonic case for the multidimensional Riemann solver. The majority of attention is lavished upon the subsonic case when designing multidimensional Riemann solvers. To bring the self-similarity to the forefront, let us pick similarity variables in two-dimensions. They are

$\tilde{\xi}=\frac{x}{t} \quad ; \quad \tilde{\psi}=\frac{y}{t}$

Notice that $(\tilde{\xi}, \tilde{\psi})$ have units of speed; mnemonically, they correspond most closely to $(x, y)$. Specifying $(\tilde{\xi}, \tilde{\psi})$ picks out a characteristic line in two space dimensions and one time dimension. In self-similar variables we have

$$
\mathbf{U}(x, y, t)=\tilde{\mathbf{U}}(\tilde{\xi}, \tilde{\psi}) \quad ; \quad \mathbf{F}(x, y, t)=\tilde{\mathbf{F}}(\tilde{\xi}, \tilde{\psi}) \quad ; \quad \mathbf{G}(x, y, t)=\tilde{\mathbf{G}}(\tilde{\xi}, \tilde{\psi})
$$

The conservation law has $\mathrm{N}$-components and is written in two dimensions as

$$
\frac{\partial \mathbf{U}}{\partial t}+\frac{\partial \mathbf{F}}{\partial x}+\frac{\partial \mathbf{G}}{\partial y}=0
$$

In similarity variables, the above equation becomes

$$
\frac{\partial(\tilde{\mathbf{F}}-\tilde{\xi} \tilde{\mathbf{U}})}{\partial \tilde{\xi}}+\frac{\partial(\tilde{\mathbf{G}}-\tilde{\psi} \tilde{\mathbf{U}})}{\partial \tilde{\psi}}+2 \tilde{\mathbf{U}}=0
$$

The above equation shows us that even in multiple dimensions, enforcing a shock jump is tantamount to enforcing the continuity of the Lagrangian fluxes across the surface of the discontinuity. In that sense, there is a fundamental connection between the formulation developed here and the formulation in Balsara [16] and Balsara \& Dumbser [17].

It is a simple problem in computational geometry to find the centroid of the wave model in Fig. 4; we denote it by $\kappa=\left(\tilde{\xi}_{c}, \tilde{\psi}_{c}\right)$. For the sake of convenience, we make the transformation of variables

$$
\xi \equiv \tilde{\xi}-\tilde{\xi}_{c} \quad ; \quad \psi \equiv \tilde{\psi}-\tilde{\psi}_{c}
$$

We call $(\xi, \psi)$ the shifted variables because they have been shifted by a velocity $\left(\tilde{\xi}_{c}, \tilde{\psi}_{c}\right)$ relative to the unshifted variables $(\tilde{\xi}, \tilde{\psi})$. The coordinates of the pentagon $P_{1} P_{2} P_{3} P_{4} P_{5}$ shown in 
Fig. 4 are the shifted coordinates; please compare Fig. 4 to Fig. 2 to appreciate the difference. A convex wave model is essential to this formulation because it ensures that the centroid $\kappa$ lies within the wave model. Following Balsara [16] we find it very beneficial to expand the states and fluxes in the shifted, self-similar coordinates. We now expand the strongly-interacting state in the shifted similarity variables. (From here on, we will refer to the shifted similarity variables as just the similarity variables, unless further specification is needed.)

$$
\tilde{\mathbf{U}}(\xi, \psi)=\overline{\mathbf{U}}+\mathbf{U}_{\xi} \xi+\mathbf{U}_{\psi} \psi
$$

The fluxes can be represented via similarity variables in three alternative ways. The $\mathrm{x}$-flux can be written in similarity variables as

$$
\tilde{\mathbf{F}}(\xi, \psi)=\overline{\mathbf{F}}+\mathbf{F}_{\xi} \xi+\mathbf{F}_{\psi} \psi
$$

As an alternative, we can make a further linearization around $\overline{\mathbf{U}}$ and write the $\mathrm{x}$-flux in selfsimilarity variables as

$\tilde{\mathbf{F}}(\xi, \psi)=\mathbf{F}(\overline{\mathbf{U}})+\overline{\mathbf{A}}(\tilde{\mathbf{U}}(\xi, \psi)-\overline{\mathbf{U}}) \quad$ with $\quad \overline{\mathbf{A}}=\frac{\partial \mathbf{F}(\overline{\mathbf{U}})}{\partial \overline{\mathbf{U}}}$

We can also, alternatively, use a Zha-Bilgen [70] type flux vector splitting to write (here $\overline{\mathrm{P}}$ is the pressure obtained from the state $\overline{\mathbf{U}}$ )

$$
\begin{aligned}
& \tilde{\mathbf{F}}(\xi, \psi)=\overline{\mathrm{v}}_{x}\left(\overline{\mathbf{U}}+\mathbf{U}_{\xi} \xi+\mathbf{U}_{\psi} \psi\right)+\left(0, \overline{\mathrm{P}}, 0,0, \overline{\mathrm{v}}_{x} \overline{\mathrm{P}}\right)^{T} \\
& \text { where } \quad \overline{\mathrm{v}}_{x} \equiv(\overline{\mathbf{U}})_{2} /(\overline{\mathbf{U}})_{1} \text { and } \overline{\mathrm{P}} \equiv(\gamma-1)\left[(\overline{\mathbf{U}})_{5}-\frac{1}{2}\left((\overline{\mathbf{U}})_{2}^{2}+(\overline{\mathbf{U}})_{3}^{2}+(\overline{\mathbf{U}})_{4}^{2}\right) /(\overline{\mathbf{U}})_{1}\right]
\end{aligned}
$$

The above flux vector splitting is specific to the Euler equations with $\gamma$ as the polytropic index. Here $(\overline{\mathbf{U}})_{1}$ is the density; $(\overline{\mathbf{U}})_{2},(\overline{\mathbf{U}})_{3}$ and $(\overline{\mathbf{U}})_{4}$ are the $\mathrm{x}-, \mathrm{y}$ - and z-momentum densities; and $(\overline{\mathbf{U}})_{5}$ is the energy density. For Euler flow such flux vector splittings are known to work well. For MHD, we use this paper as an opportunity to explore whether such a flux vector splitting might work successfully. Analogously to the x-flux, the y-flux can be written in similarity variables as

$$
\tilde{\mathbf{G}}(\xi, \psi)=\overline{\mathbf{G}}+\mathbf{G}_{\xi} \xi+\mathbf{G}_{\psi} \psi
$$

Alternatively, we can make a further linearization and write the y-flux in self-similarity variables as

$$
\tilde{\mathbf{G}}(\xi, \psi)=\mathbf{G}(\overline{\mathbf{U}})+\overline{\mathbf{B}}(\tilde{\mathbf{U}}(\xi, \psi)-\overline{\mathbf{U}}) \quad \text { with } \quad \overline{\mathbf{B}}=\frac{\partial \mathbf{G}(\overline{\mathbf{U}})}{\partial \overline{\mathbf{U}}}
$$


We can also, alternatively, use a Zha-Bilgen [70] type flux vector splitting to write (here $\overline{\mathrm{P}}$ is again the pressure obtained from the state $\overline{\mathbf{U}}$ )

$\tilde{\mathbf{G}}(\xi, \psi)=\overline{\mathrm{v}}_{y}\left(\overline{\mathbf{U}}+\mathbf{U}_{\xi} \xi+\mathbf{U}_{\psi} \psi\right)+\left(0,0, \overline{\mathrm{P}}, 0, \overline{\mathrm{v}}_{y} \overline{\mathrm{P}}\right)^{T} \quad$ where $\quad \overline{\mathrm{v}}_{y} \equiv(\overline{\mathbf{U}})_{3} /(\overline{\mathbf{U}})_{1}$

Eqns. (7c) and (8c) are specific to the Euler equations, but they could perhaps be generalized to other systems. Notice that eqns. (7b) and (8b) have different import from eqns. (7a) and (8a). The same is true for eqns. (7c) and (8c). We will see in the next sub-section that eqns. (7a) and (7a) result in a certain kind of formulation for the multidimensional Riemann problem. Eqns. (7b) and (8b) result in a slightly different kind of formulation. Eqns. (7c) and (8c), however, yield the simplest formulation.

The state $\overline{\mathbf{U}}$ in eqn. (6) can be obtained by integrating the fluxes over the boundaries of the wave model, as shown in Wendroff [68], Balsara [4,5,16] and Vides, Nkonga \& Audit [67]. The other terms in eqns. (6) to (8) can be obtained in several different ways. One very prominent way is via Galerkin projection as shown in Balsara [16] and Balsara \& Dumbser [17]. Another approach from Vides, Nkonga \& Audit [67] is to directly impose the shock jump condition at the boundary of the wave model. So far, the latter approach has only been made to yield a constant strongly interacting state. In this paper we draw on the self-similar formulation of Balsara [16] to show that shock jumps can be imposed while retaining additional sub-structure in the stronglyinteracting state.

The philosophy that is specific to this paper is that we should take the boundary of the strongly-interacting state quite literally. In that case, one-dimensional shock-jumps are to be enforced normal to each planar wavefront that bounds the multidimensional wave model. Since each flat boundary of the wave model in Fig. 4 has two states associated with it when a onedimensional HLLC Riemann solver is used, we will usually have two such shock jumps at each wavefront. If a larger wave model is used as shown in Fig. 5 then, of course, one will have more shock jumps within each wavefront. Let the area of the wave model in Fig. 4 be denoted by $\Omega$ and let its boundary be denoted by $\partial \Omega$. Realize that this boundary is made up of $I_{\max }$ individual flat panels which we refer to as wavefronts. The shock jump at any point $(\xi, \psi)$ on the $i^{\text {th }}$ panel of the wave model can be written as

$$
\eta_{i x} \mathbf{F}\left(\xi_{-}, \psi_{-}\right)+\eta_{i y} \mathbf{G}\left(\xi_{-}, \psi_{-}\right)-S_{i} \mathbf{U}\left(\xi_{-}, \psi_{-}\right)=\eta_{i x} \mathbf{F}^{*}\left(\xi_{+}, \psi_{+}\right)+\eta_{i y} \mathbf{G}^{*}\left(\xi_{+}, \psi_{+}\right)-S_{i} \mathbf{U}^{*}\left(\xi_{+}, \psi_{+}\right)
$$

The fluxes $\mathbf{F}\left(\xi_{-}, \psi_{-}\right)$and $\mathbf{G}\left(\xi_{-}, \psi_{-}\right)$and state $\mathbf{U}\left(\xi_{-}, \psi_{-}\right)$pertain to a location that is just inside the wave model while the fluxes $\mathbf{F}^{*}\left(\xi_{+}, \psi_{+}\right)$and $\mathbf{G}^{*}\left(\xi_{+}, \psi_{+}\right)$and the state $\mathbf{U}^{*}\left(\xi_{+}, \psi_{+}\right)$pertain to a corresponding location that is just outside the wave model. The fluxes and states that are just outside the wave model are known to us from our solution of the one-dimensional Riemann problems, please see Fig. 1. Since eqns. (6), (7a) and (8a) constitute the most general expression 
of our formulation, we may take our vector of unknowns to be $\mathbf{V}=\left(\mathbf{U}_{\xi}, \mathbf{U}_{\psi}, \overline{\mathbf{F}}, \mathbf{F}_{\xi}, \mathbf{F}_{\psi}, \overline{\mathbf{G}}, \mathbf{G}_{\xi}, \mathbf{G}_{\psi}\right)^{T}$. In general, the amount of information in $\mathbf{V}$ is insufficient to satisfy the shock jump condition at all points on the boundary of the self-similarly expanding wave model. Realize too that the polygonal boundary of the expanding wave model is indeed an idealized construction and that the strongly-interacting state does not need to conform to the shape of the wave model. Consequently, the jump conditions in eqn. (9) are also an expression of the continuity of Lagrangian fluxes across a moving boundary. We can aspire to satisfy the continuity of the Lagrangian fluxes across the multidimensional wave model as best as possible by minimizing

$$
\sum_{i=1}^{I_{\max }} \int_{\partial \Omega_{i}}\left[\eta_{i x} \mathbf{F}\left(\xi_{-}, \psi_{-}\right)+\eta_{i y} \mathbf{G}\left(\xi_{-}, \psi_{-}\right)-S_{i} \mathbf{U}\left(\xi_{-}, \psi_{-}\right)-\eta_{i x} \mathbf{F}^{*}\left(\xi_{+}, \psi_{+}\right)-\eta_{i y} \mathbf{G}^{*}\left(\xi_{+}, \psi_{+}\right)+S_{i} \mathbf{U}^{*}\left(\xi_{+}, \psi_{+}\right)\right]^{2} d l_{i}
$$

Here $d l_{i}$ is a line element in the $i^{\text {th }}$ panel/wavefront $\partial \Omega_{i}$ of the wave model $\Omega$. The above equation expresses the least squares minimization that is built into our approach.

Minimizing eqn. (10) is tantamount to minimizing it within each of the panels/wavefronts of the multidimensional wave model. Depending on the one-dimensional Riemann solver being used, and also depending also on the form of the multidimensional wave model being used, we could have multiple constant states immediately outside each of the panels of the wave model. For example, in Fig. 4, each side panel has two constant states because we are using a onedimensional HLLC Riemann solver. Let $l_{i}^{j}$ be the segment that corresponds to the $j^{\text {th }}$ constant state in the $i^{\text {th }}$ panel/wavefront. The least squares minimization then gives us

$l_{i}^{j}\left[\eta_{i x} \mathbf{F}\left(\xi_{i}^{j}, \psi_{i}^{j}\right)+\eta_{i y} \mathbf{G}\left(\xi_{i}^{j}, \psi_{i}^{j}\right)-S_{i} \mathbf{U}\left(\xi_{i}^{j}, \psi_{i}^{j}\right)\right]=l_{i}^{j}\left[\eta_{i x} \mathbf{F}^{*}\left(\xi_{i}^{j}, \psi_{i}^{j}\right)+\eta_{i y} \mathbf{G}^{*}\left(\xi_{i}^{j}, \psi_{i}^{j}\right)-S_{i} \mathbf{U}^{*}\left(\xi_{i}^{j}, \psi_{i}^{j}\right)\right]$

Here $\left(\xi_{i}^{j}, \psi_{i}^{j}\right)$ is the center point of the segment $l_{i}^{j}$; corresponding to a mid-point quadrature formula. In eqn. (11), $\mathbf{F}\left(\xi_{i}^{j}, \psi_{i}^{j}\right), \mathbf{G}\left(\xi_{i}^{j}, \psi_{i}^{j}\right)$ and $\mathbf{U}\left(\xi_{i}^{j}, \psi_{i}^{j}\right)$ are to be evaluated from within the wave model using the unknowns from the vector $\mathbf{V}$. By contrast, $\mathbf{F}^{*}\left(\xi_{i}^{j}, \psi_{i}^{j}\right), \mathbf{G}^{*}\left(\xi_{i}^{j}, \psi_{i}^{j}\right)$ and $\mathbf{U}^{*}\left(\xi_{i}^{j}, \psi_{i}^{j}\right)$ are available from the one-dimensional Riemann problems from outside the wave model. In the above equation we have chosen a very simplified quadrature, namely the midpoint rule, because a more intricate quadrature would only increase the computational cost without increasing the order of the method. The above equation can be viewed either as a weighted version of the weak form of the shock jump conditions or as a way of matching Lagrangian fluxes across a moving boundary. 
Let us substantiate our thinking by focusing on the first face, formed by segment $P_{1} P_{2}$, in Fig. 4. It is made up of two sub-segments $P_{1} C_{1}$ and $C_{1} P_{2}$, so let us identify the midpoints of each of those sub-segments as $\left(\xi_{P 1 C 1}, \psi_{P 1 C 1}\right)$ and $\left(\xi_{C 1 P 2}, \psi_{C 1 P 2}\right)$. Let be the lengths $l_{P 1 C 1}$ and $l_{C 1 P 2}$ of these two sub-segments. The coordinates of these midpoints, as well as their lengths, are very easy to obtain using the notation that we have set up in Fig. 4. The shock jumps (normal to the boundary of the wave model) at each of those midpoints can now be written explicitly as

$$
l_{P 1 C 1}\left[\eta_{1 x} \mathbf{F}\left(\xi_{P 1 C 1}, \psi_{P 1 C 1}\right)+\eta_{1 y} \mathbf{G}\left(\xi_{P 1 C 1}, \psi_{P 1 C 1}\right)-S_{1} \mathbf{U}\left(\xi_{P 1 C 1}, \psi_{P 1 C 1}\right)\right]=l_{P 1 C 1}\left[\eta_{1 x} \mathbf{F}_{1}^{*-}+\eta_{1 y} \mathbf{G}_{1}^{*-}-S_{1} \mathbf{U}_{1}^{*-}\right]
$$

and

$$
l_{C 1 P 2}\left[\eta_{1 x} \mathbf{F}\left(\xi_{C 1 P 2}, \psi_{C 1 P 2}\right)+\eta_{1 y} \mathbf{G}\left(\xi_{C 1 P 2}, \psi_{C 1 P 2}\right)-S_{1} \mathbf{U}\left(\xi_{C 1 P 2}, \psi_{C 1 P 2}\right)\right]=l_{C 1 P 2}\left[\eta_{1 x} \mathbf{F}_{1}^{*+}+\eta_{1 y} \mathbf{G}_{1}^{*+}-S_{1} \mathbf{U}_{1}^{*+}\right]
$$

The lengths of the sub-segments, $l_{P 1 C 1}$ and $l_{C 1 P 2}$, within the first panel/wavefront serve as weights when constructing the least squares matrix. Analogous jump conditions can be written for the other four faces of the multidimensional wave model in Fig. 4. When " $I_{\max }$ " states come together at a vertex, and if we use an HLLC Riemann solver as a building block, we will have " $2 I_{\max }$ " such jump conditions. When the above two jump conditions, as well as their analogues in all the other faces of the multidimensional wave model, are enforced we have an overdetermined system. We show that the least squares procedure is always capable of giving us a solution in that circumstance. Another way to understand the above two equations is that in the Galerkin formulation of Balsara [16], the lengths of the sides of the wave model play a role in the integration over the faces of the wave model. Weighting the equations with the lengths of the sub-segments also reflects the insights we have derived from the Galerkin formulation.

There are various ways in which the shock jump conditions can be imposed with a view to retaining sub-structure and we present some of the choices in the next three sub-sections.

\section{III.b) Imposing Jump Conditions; The Easiest Option}

We begin by describing the easiest option. Using eqns. (7c) and (8c) allows us to write the jumps at the midpoints of the relevant segments in the easiest way. The simplest option, therefore, consists of using eqns. (7c) and (8c) along with eqn. (6). In that case, the linear terms in eqn. (6) are the only unknowns, but notice that the flux vector splitting has linearized the problem. When eqns. (6), (7c) and (8c) are substituted in eqns. (12) and (13), we get 


$$
\begin{aligned}
& l_{P 1 C 1}\left[\left(\eta_{1 x} \overline{\mathrm{v}}_{x}+\eta_{1 y} \overline{\mathrm{v}}_{y}-S_{1}\right) \xi_{P 1 C 1} \mathbf{U}_{\xi}+\left(\eta_{1 x} \overline{\mathrm{v}}_{x}+\eta_{1 y} \overline{\mathrm{v}}_{y}-S_{1}\right) \psi_{P 1 C 1} \mathbf{U}_{\psi}\right]= \\
& l_{P 1 C 1}\left[\eta_{1 x}\left(\mathbf{F}_{1}^{*-}-\overline{\mathrm{v}}_{x} \overline{\mathbf{U}}-\left(0, \overline{\mathrm{P}}, 0,0, \overline{\mathrm{v}}_{x} \overline{\mathrm{P}}\right)^{T}\right)+\eta_{1 y}\left(\mathbf{G}_{1}^{*-}-\overline{\mathrm{v}}_{y} \overline{\mathbf{U}}-\left(0,0, \overline{\mathrm{P}}, 0, \overline{\mathrm{v}}_{y} \overline{\mathrm{P}}\right)^{T}\right)-S_{1}\left(\mathbf{U}_{1}^{*-}-\overline{\mathbf{U}}\right)\right]
\end{aligned}
$$

and

$$
\begin{aligned}
& l_{C 1 P 2}\left[\left(\eta_{1 x} \overline{\mathrm{v}}_{x}+\eta_{1 y} \overline{\mathrm{v}}_{y}-S_{1}\right) \xi_{C 1 P 2} \mathbf{U}_{\xi}+\left(\eta_{1 x} \overline{\mathrm{v}}_{x}+\eta_{1 y} \overline{\mathrm{v}}_{y}-S_{1}\right) \psi_{C 1 P 2} \mathbf{U}_{\psi}\right]= \\
& l_{C 1 P 2}\left[\eta_{1 x}\left(\mathbf{F}_{1}^{*+}-\overline{\mathrm{v}}_{x} \overline{\mathbf{U}}-\left(0, \overline{\mathrm{P}}, 0,0, \overline{\mathrm{v}}_{x} \overline{\mathrm{P}}\right)^{T}\right)+\eta_{1 y}\left(\mathbf{G}_{1}^{*+}-\overline{\mathrm{v}}_{y} \overline{\mathbf{U}}-\left(0,0, \overline{\mathrm{P}}, 0, \overline{\mathrm{v}}_{y} \overline{\mathrm{P}}\right)^{T}\right)-S_{1}\left(\mathbf{U}_{1}^{*+}-\overline{\mathbf{U}}\right)\right]
\end{aligned}
$$

Please notice that eqns. (14) and (15) are identical to eqns. (12) and (13).

Analogous equations can be written for the other four faces of the multidimensional wave model in Fig. 4. Notice that eqns. (14) and (15) and their other analogues only have two unknowns, i.e. $\mathbf{U}_{\xi}$ and $\mathbf{U}_{\psi}$. Thus we again have an overdetermined set of eight equations in two unknowns, which can be treated via a least squares algorithm. Quite favorably, the size of the least squares problem does not depend on $N$, the number of components of the hyperbolic system. Thus we favor this solution methodology for Euler flow. In this paper we present preliminary evidence that it might also extend to MHD flow.

Eqns. (14) and (15) require that the fluxes $\mathbf{F}_{1}^{*_{-}}, \mathbf{G}_{1}^{*_{-}}, \mathbf{F}_{1}^{*+}$ and $\mathbf{G}_{1}^{*_{+}}$are defined in a global frame. In practice, they may be obtained in a rotated frame so that we might obtain the HLLC/HLLD fluxes in a frame that is aligned with the direction of the one-dimensional Riemann problem. (For the HLLC Riemann solver, this task is explicitly done in Appendix A of Balsara, Dumbser \& Abgrall [15]. For the HLLD Riemann solver, this is explicitly presented in Appendix B of Balsara \& Dumbser [17].) For the first face in Fig. 1, this would be a frame with the Riemann problem solved in the $\mathbf{t}_{1}$ direction, with the interface between the zones being in the $\mathbf{n}_{1}$ direction. We would then have $\tilde{\mathbf{F}}_{\mathbf{t} 1}^{*-}=-n_{1 y} \mathbf{F}_{1}^{*-}+n_{1 x} \mathbf{G}_{1}^{*-}$ and $\tilde{\mathbf{G}}_{\mathbf{n} 1}^{*-}=n_{1 x} \mathbf{F}_{1}^{*-}+n_{1 y} \mathbf{G}_{1}^{*-}$, with analogous expressions for $\tilde{\mathbf{F}}_{\mathbf{t} 1}^{*+}$ and $\tilde{\mathbf{G}}_{\mathbf{n} 1}^{*+}$. The expressions that are equivalent to eqns. (14) and (15) would then be

$$
l_{P 1 C 1}\left[\xi_{P 1 C 1} \mathbf{U}_{\xi}+\psi_{P 1 C 1} \mathbf{U}_{\psi}\right]=\frac{l_{P 1 C 1}}{\left(\eta_{1 x} \overline{\mathrm{v}}_{x}+\eta_{1 y} \overline{\mathbf{v}}_{y}-S_{1}\right)}\left[\begin{array}{l}
\left(\boldsymbol{\eta}_{1} \bullet \mathbf{t}_{1}\right) \tilde{\mathbf{F}}_{\mathbf{1}}^{*-}+\left(\boldsymbol{\eta}_{1} \cdot \mathbf{n}_{1}\right) \tilde{\mathbf{G}}_{\mathbf{n} 1}^{*-}-\eta_{1 x}\left(\overline{\mathrm{v}}_{x} \overline{\mathbf{U}}+\left(0, \overline{\mathrm{P}}, 0,0, \overline{\mathrm{v}}_{x} \overline{\mathrm{P}}\right)^{T}\right) \\
-\eta_{1 y}\left(\overline{\mathrm{v}}_{y} \overline{\mathbf{U}}^{T}+\left(0,0, \overline{\mathrm{P}}, 0, \overline{\mathrm{v}}_{y} \overline{\mathrm{P}}\right)^{T}\right)-S_{1}\left(\mathbf{U}_{1}^{*-}-\overline{\mathbf{U}}\right)
\end{array}\right]
$$

and 


$$
l_{C 1 P 2}\left[\xi_{C 1 P 2} \mathbf{U}_{\xi}+\psi_{C 1 P 2} \mathbf{U}_{\psi}\right]=\frac{l_{C 1 P 2}}{\left(\eta_{1 x} \overline{\mathbf{v}}_{x}+\eta_{1 y} \overline{\mathbf{v}}_{y}-S_{1}\right)}\left[\begin{array}{l}
\left(\boldsymbol{\eta}_{1} \cdot \mathbf{t}_{1}\right) \tilde{\mathbf{F}}_{\mathbf{t} 1}^{*+}+\left(\boldsymbol{\eta}_{1} \cdot \mathbf{n}_{1}\right) \tilde{\mathbf{G}}_{\mathbf{n} 1}^{*+}-\eta_{1 x}\left(\overline{\mathbf{v}}_{x} \overline{\mathbf{U}}+\left(0, \overline{\mathrm{P}}, 0,0, \overline{\mathrm{v}}_{x} \overline{\mathrm{P}}\right)^{T}\right) \\
-\eta_{1 y}\left(\overline{\mathbf{v}}_{y} \overline{\mathbf{U}}+\left(0,0, \overline{\mathrm{P}}, 0, \overline{\mathrm{v}}_{y} \overline{\mathrm{P}}\right)^{T}\right)-S_{1}\left(\mathbf{U}_{1}^{*+}-\overline{\mathbf{U}}\right)
\end{array}\right]
$$

In eqns. (16) and (17) we notice that the coefficients for all the components of $\mathbf{U}_{\xi}$ and $\mathbf{U}_{\psi}$ are constant. As a result, the least squares problem reduces to the inversion of a $2 \times 2$ matrix, making the problem for obtaining $\mathbf{U}_{\xi}$ and $\mathbf{U}_{\psi}$ very simple. We have simplified eqns. (16) and (17) for computer implementation, by dividing them out with $\left(\eta_{1 x} \overline{\mathrm{v}}_{x}+\eta_{1 y} \overline{\mathrm{v}}_{y}-S_{1}\right)$. Please note that the right hand sides of eqns. (16) and (17) are not matrix expressions. Appendix A describes the matrix-based formulation of the least squares minimization process. Explicit construction of the $2 \times 2$ matrix is also presented in Appendix A.

The $2 \times 2$ matrix that is presented in Appendix A can always be inverted. An analogous simplification was shown to exist in the Galerkin formulation, see Section VI of Balsara [16] or Section III.b of Balsara \& Dumbser [17] where it was proved that the Galerkin formulation always guarantees the existence of a solution for the linear variation in eqn. (6). In Appendix B of this paper we prove that the least squares procedure described in this Sub-section provides a similar guarantee that a solution for $\mathbf{U}_{\xi}$ and $\mathbf{U}_{\psi}$ can always be found. The proof requires that $\left(\eta_{1 x} \overline{\mathrm{v}}_{x}+\eta_{1 y} \overline{\mathrm{v}}_{y}-S_{1}\right)$ is non-zero, which is very likely if the wave model represents the boundary of the largest one-dimensional speeds. It is, however, not guaranteed for the wave model described in Section II. Use of the wave model from Section II.2 and Appendix B of Balsara, Dumbser \& Abgrall [15] would indeed guarantee that $\left(\eta_{1 x} \overline{\mathrm{v}}_{x}+\eta_{1 y} \overline{\mathrm{v}}_{y}-S_{1}\right)$ is non-zero.

\section{III.c) Imposing Jump Conditions; Retaining More Degrees of Freedom}

The next option consists of using eqns. (7a) and (8a). Recall that the state $\overline{\mathbf{U}}$ in eqn. (6) is known. Thus eqns. (6), (7a) and (8a) constitute three equations with eight unknowns. The eight unknowns are the fluxes $\overline{\mathbf{F}}$ and $\overline{\mathbf{G}}$ along with the linear variations of the state, i.e. $\mathbf{U}_{\xi}$ and $\mathbf{U}_{\psi}$, and the linear variation of the two fluxes, i.e. $\mathbf{F}_{\xi}, \mathbf{F}_{\psi}, \mathbf{G}_{\xi}$ and $\mathbf{G}_{\psi}$. When eqns. (6), (7a) and (8a) are substituted in eqns. (12) and (13) we find

$$
\begin{aligned}
& l_{P 1 C 1}\left[\eta_{1 x} \overline{\mathbf{F}}+\left(\eta_{1 x} \xi_{P 1 C 1}\right) \mathbf{F}_{\xi}+\left(\eta_{1 x} \psi_{P 1 C 1}\right) \mathbf{F}_{\psi}+\eta_{1 y} \overline{\mathbf{G}}+\left(\eta_{1 y} \xi_{P 1 C 1}\right) \mathbf{G}_{\xi}+\left(\eta_{1 y} \psi_{P 1 C 1}\right) \mathbf{G}_{\psi}-\left(S_{1} \xi_{P 1 C 1}\right) \mathbf{U}_{\xi} \xi_{-}\left(S_{1} \psi_{P 1 C 1}\right) \mathbf{U}_{\psi}\right] \\
& =l_{P 1 C 1}\left[\eta_{1 x} \mathbf{F}_{1}^{*-}+\eta_{1 y} \mathbf{G}_{1}^{*_{-}-}-S_{1}\left(\mathbf{U}_{1}^{*-}-\overline{\mathbf{U}}\right)\right]
\end{aligned}
$$

and 


$$
\begin{aligned}
& l_{C 1 P 2}\left[\eta_{1 x} \overline{\mathbf{F}}+\left(\eta_{1 x} \xi_{C 1 P 2}\right) \mathbf{F}_{\xi}+\left(\eta_{1 x} \psi_{C 1 P 2}\right) \mathbf{F}_{\psi}+\eta_{1 y} \overline{\mathbf{G}}+\left(\eta_{1 y} \xi_{C 1 P 2}\right) \mathbf{G}_{\xi}+\left(\eta_{1 y} \psi_{C 1 P 2}\right) \mathbf{G}_{\psi}-\left(S_{1} \xi_{C 1 P 2}\right) \mathbf{U}_{\xi} \xi-\left(S_{1} \psi_{C 1 P 2}\right) \mathbf{U}_{\psi}\right] \\
& =l_{C 1 P 2}\left[\eta_{1 x} \mathbf{F}_{1}^{*+}+\eta_{1 y} \mathbf{G}_{1}^{*+}-S_{1}\left(\mathbf{U}_{1}^{*+}-\overline{\mathbf{U}}\right)\right]
\end{aligned}
$$

Similar equations can be asserted in all the wavefronts that constitute the multidimensional wave model. As long as the wave model has four or more faces, it leads to a viable least squares problem. (If only three zones come together at a vertex, the least squares problem cannot be solved per se. In that case, one either can resort to making an angular bisection of the zones or increasing the number of quadrature points within each wavefront.)

If we choose to simplify the problem by asserting $\overline{\mathbf{F}}=\mathbf{F}(\overline{\mathbf{U}})$ and $\overline{\mathbf{G}}=\mathbf{G}(\overline{\mathbf{U}})$ then eqns. (18) and (19) reduce to

$$
\begin{aligned}
& l_{P 1 C 1}\left[\left(\eta_{1 x} \xi_{P 1 C 1}\right) \mathbf{F}_{\xi}+\left(\eta_{1 x} \psi_{P 1 C 1}\right) \mathbf{F}_{\psi}+\left(\eta_{1 y} \xi_{P 1 C 1}\right) \mathbf{G}_{\xi}+\left(\eta_{1 y} \psi_{P 1 C 1}\right) \mathbf{G}_{\psi}-\left(S_{1} \xi_{P 1 C 1}\right) \mathbf{U}_{\xi}-\left(S_{1} \psi_{P 1 C 1}\right) \mathbf{U}_{\psi}\right]= \\
& l_{P 1 C 1}\left[\eta_{1 x}\left(\mathbf{F}_{1}^{*-}-\mathbf{F}(\overline{\mathbf{U}})\right)+\eta_{1 y}\left(\mathbf{G}_{1}^{*-}-\mathbf{G}(\overline{\mathbf{U}})\right)-S_{1}\left(\mathbf{U}_{1}^{*-}-\overline{\mathbf{U}}\right)\right]
\end{aligned}
$$

and

$$
\begin{aligned}
& l_{C 1 P 2}\left[\left(\eta_{1 x} \xi_{C 1 P 2}\right) \mathbf{F}_{\xi}+\left(\eta_{1 x} \psi_{C 1 P 2}\right) \mathbf{F}_{\psi}+\left(\eta_{1 y} \xi_{C 1 P 2}\right) \mathbf{G}_{\xi}+\left(\eta_{1 y} \psi_{C 1 P 2}\right) \mathbf{G}_{\psi}-\left(S_{1} \xi_{C 1 P 2}\right) \mathbf{U}_{\xi}-\left(S_{1} \psi_{C 1 P 2}\right) \mathbf{U}_{\psi}\right]= \\
& l_{C 1 P 2}\left[\eta_{1 x}\left(\mathbf{F}_{1}^{*+}-\mathbf{F}(\overline{\mathbf{U}})\right)+\eta_{1 y}\left(\mathbf{G}_{1}^{*+}-\mathbf{G}(\overline{\mathbf{U}})\right)-S_{1}\left(\mathbf{U}_{1}^{*+}-\overline{\mathbf{U}}\right)\right]
\end{aligned}
$$

Because of the reduction in the number of unknowns, this system is solvable by a least squares procedure in all circumstances; i.e., even when only three zones come together at a vertex. In this case, we are only solving for the linear variations. I.e. our unknowns are the linear variations of the state, i.e. $\mathbf{U}_{\xi}$ and $\mathbf{U}_{\psi}$, and the linear variation of the two fluxes, i.e. $\mathbf{F}_{\xi}, \mathbf{F}_{\psi}, \mathbf{G}_{\xi}$ and $\mathbf{G}_{\psi}$. The least square procedure results in a $6 \times 6$ matrix.

In Section III.d of Balsara [16] we proved that the problem described in this Sub-section always results in a solvable system when Galerkin projection is used. In Appendix C of this paper we show that eqns. (20) and (21) also result in a solvable $6 \times 6$ matrix system when least squares projection is used. As in this Sub-section, the coefficients of the linear system in Balsara [16] also depended on the geometry of the wave model and the geometry of the mesh.

\section{III.d) Imposing Jump Conditions; Coupling Fluxes and States via the Characteristic Matrices}


While the previous Sub-section tried to allow for independent variation in the fluxes and states, we realize that they are indeed coupled. The third option consists of using eqns. (7b) and (8b) along with eqn. (6). A solution can be sought in two possible flavors. The first flavor of solution treats the fluxes $\overline{\mathbf{F}}$ and $\overline{\mathbf{G}}$ as unknowns and it also treats the linear variation of the solution vector, $\mathbf{U}_{\xi}$ and $\mathbf{U}_{\psi}$, as unknowns. We still retain $\overline{\mathbf{A}}=\mathbf{A}(\overline{\mathbf{U}})$ and $\overline{\mathbf{B}}=\mathbf{B}(\overline{\mathbf{U}})$. In that case, the least squares problem becomes

$$
\begin{aligned}
& l_{P 1 C 1}\left[\eta_{1 x} \overline{\mathbf{F}}+\eta_{1 y} \overline{\mathbf{G}}+\xi_{P 1 C 1}\left(\eta_{1 x} \overline{\mathbf{A}}+\eta_{1 y} \overline{\mathbf{B}}-S_{1} \mathbf{I}\right) \mathbf{U}_{\xi}+\psi_{P 1 C 1}\left(\eta_{1 x} \overline{\mathbf{A}}+\eta_{1 y} \overline{\mathbf{B}}-S_{1} \mathbf{I}\right) \mathbf{U}_{\psi}\right]= \\
& l_{P 1 C 1}\left[\eta_{1 x} \mathbf{F}_{1}^{*_{-}}+\eta_{1 y} \mathbf{G}_{1}^{*_{-}}-S_{1}\left(\mathbf{U}_{1}^{*-}-\overline{\mathbf{U}}\right)\right]
\end{aligned}
$$

and

$$
\begin{aligned}
& l_{C 1 P 2}\left[\eta_{1 x} \overline{\mathbf{F}}+\eta_{1 y} \overline{\mathbf{G}}+\xi_{C 1 P 2}\left(\eta_{1 x} \overline{\mathbf{A}}+\eta_{1 y} \overline{\mathbf{B}}-S_{1} \mathbf{I}\right) \mathbf{U}_{\xi}+\psi_{C 1 P 2}\left(\eta_{1 x} \overline{\mathbf{A}}+\eta_{1 y} \overline{\mathbf{B}}-S_{1} \mathbf{I}\right) \mathbf{U}_{\psi}\right]= \\
& l_{C 1 P 2}\left[\eta_{1 x} \mathbf{F}_{1}^{*+}+\eta_{1 y} \mathbf{G}_{1}^{*+}-S_{1}\left(\mathbf{U}_{1}^{*+}-\overline{\mathbf{U}}\right)\right]
\end{aligned}
$$

In this form, the equations have $4 N$ unknowns, making the least squares problem at least a little challenging, especially for larger hyperbolic systems. It would, eventually, require the inversion of a $4 N \times 4 N$ matrix, which can be computationally expensive. Next, we seek a simplification.

The second flavor of solution linearizes even further around the mean state, so that we have $\overline{\mathbf{F}}=\mathbf{F}(\overline{\mathbf{U}})$ and $\overline{\mathbf{G}}=\mathbf{G}(\overline{\mathbf{U}})$. In that case, the linear terms in eqn. (6) are the only unknowns. The corresponding jump conditions can now be obtained as

$$
\begin{aligned}
& l_{P 1 C 1}\left[\xi_{P 1 C 1}\left(\eta_{1 x} \overline{\mathbf{A}}+\eta_{1 y} \overline{\mathbf{B}}-S_{1} \mathbf{I}\right) \mathbf{U}_{\xi}+\psi_{P 1 C 1}\left(\eta_{1 x} \overline{\mathbf{A}}+\eta_{1 y} \overline{\mathbf{B}}-S_{1} \mathbf{I}\right) \mathbf{U}_{\psi}\right]= \\
& l_{P 1 C 1}\left[\eta_{1 x}\left(\mathbf{F}_{1}^{*-}-\mathbf{F}(\overline{\mathbf{U}})\right)+\eta_{1 y}\left(\mathbf{G}_{1}^{*-}-\mathbf{G}(\overline{\mathbf{U}})\right)-S_{1}\left(\mathbf{U}_{1}^{*-}-\overline{\mathbf{U}}\right)\right]
\end{aligned}
$$

and

$$
\begin{aligned}
& l_{C 1 P 2}\left[\xi_{C 1 P 2}\left(\eta_{1 x} \overline{\mathbf{A}}+\eta_{1 y} \overline{\mathbf{B}}-S_{1} \mathbf{I}\right) \mathbf{U}_{\xi}+\psi_{C 1 P 2}\left(\eta_{1 x} \overline{\mathbf{A}}+\eta_{1 y} \overline{\mathbf{B}}-S_{1} \mathbf{I}\right) \mathbf{U}_{\psi}\right]= \\
& l_{C 1 P 2}\left[\eta_{1 x}\left(\mathbf{F}_{1}^{*+}-\mathbf{F}(\overline{\mathbf{U}})\right)+\eta_{1 y}\left(\mathbf{G}_{1}^{*_{+}}-\mathbf{G}(\overline{\mathbf{U}})\right)-S_{1}\left(\mathbf{U}_{1}^{*+}-\overline{\mathbf{U}}\right)\right]
\end{aligned}
$$

Thus eqns. (24) and (25) and their analogues constitute an overdetermined system of $2 N$ unknowns; though we soon show that a substantial simplification is possible. This method is beneficial because the individual components of $\mathbf{U}_{\xi}$ and $\mathbf{U}_{\psi}$ are allowed to vary in response to the characteristic matrices. In practice, eqns. (24) and (25) should be implemented into a computer code as follows: 


$$
\begin{aligned}
& l_{P 1 C 1}\left[\xi_{P 1 C 1} \mathbf{U}_{\xi}+\psi_{P 1 C 1} \mathbf{U}_{\psi}\right]= \\
& l_{P 1 C 1}\left(\eta_{1 x} \overline{\mathbf{A}}+\eta_{1 y} \overline{\mathbf{B}}-S_{1} \mathbf{I}\right)^{-1}\left[\eta_{1 x}\left(\mathbf{F}_{1}^{*-}-\mathbf{F}(\overline{\mathbf{U}})\right)+\eta_{1 y}\left(\mathbf{G}_{1}^{*-}-\mathbf{G}(\overline{\mathbf{U}})\right)-S_{1}\left(\mathbf{U}_{1}^{*-}-\overline{\mathbf{U}}\right)\right]
\end{aligned}
$$

and

$$
\begin{aligned}
& l_{C 1 P 2}\left[\xi_{C 1 P 2} \mathbf{U}_{\xi}+\psi_{C 1 P 2} \mathbf{U}_{\psi}\right]= \\
& l_{C 1 P 2}\left(\eta_{1 x} \overline{\mathbf{A}}+\eta_{1 y} \overline{\mathbf{B}}-S_{1} \mathbf{I}\right)^{-1}\left[\eta_{1 x}\left(\mathbf{F}_{1}^{*_{+}}-\mathbf{F}(\overline{\mathbf{U}})\right)+\eta_{1 y}\left(\mathbf{G}_{1}^{*_{+}}-\mathbf{G}(\overline{\mathbf{U}})\right)-S_{1}\left(\mathbf{U}_{1}^{*_{+}}-\overline{\mathbf{U}}\right)\right]
\end{aligned}
$$

The above format is more economical because it saves on the cost of inverting larger matrices. Within the $i^{t h}$ flat face of the multidimensional Riemann problem we have only to invert the $N \times N$ matrix given by $\left(\eta_{i x} \overline{\mathbf{A}}+\eta_{i y} \overline{\mathbf{B}}-S_{i} \mathbf{I}\right)$; which is not so computationally costly. In that case, the least squares problem can be reduced to the inversion of a $2 \times 2$ matrix that is not unlike the one obtained from eqns. (16) and (17) (or in fact eqns. (14) and (15)).

A little inspection shows that it is also not safe to use eqns. (24) and (25), as given, with the wave model from Section II. The reason is that the wave model is the smallest one that we can construct. As a result, speed $S_{1}$ in eqns. (24) and (25) can indeed coincide with the extremal eigenvalues of $\eta_{1 x} \overline{\mathbf{A}}+\eta_{1 y} \overline{\mathbf{B}}$, making an inversion of the matrix impossible. There is, however, a multidimensional wave model construction that is described in Section II.2 and Appendix B of Balsara, Dumbser \& Abgrall [15] which can indeed produce a larger wave model. Such a wave model can be made to ensure that the speed $S_{1}$ is always larger than the largest eigenvalue of $\eta_{1 x} \overline{\mathbf{A}}+\eta_{1 y} \overline{\mathbf{B}}$. This can be ensured for all the wavefronts that make up the multidimensional wave model. Such a choice is also consistent with entropy enforcement. This opens the door to making eqns. (24) and (25) usable. For the scalar case, we can indeed assume that $\eta_{1 x} \overline{\mathbf{A}}+\eta_{1 y} \overline{\mathbf{B}}$ is always a number that is somewhat smaller than $S_{1}$ so that $\left(\eta_{1 x} \overline{\mathbf{A}}+\eta_{1 y} \overline{\mathbf{B}}-S_{1}\right)$ is always a negative number. With this choice of $S_{i}$, the least squares solution to eqns. (24) and (25) always yields invertible $2 \times 2$ matrices for the scalar case. In fact, in the scalar limit, the left hand sides of eqns. (14) and (15) become similar to the left hand sides of eqns. (24) and (25). As a result, for the scalar case, the least squares projection can still be viewed as provably invertible for the scheme presented in this sub-section.

\section{III.e) Additional Detail}

Notice that the solution methodology relies on asserting $\overline{\mathbf{F}}=\mathbf{F}(\overline{\mathbf{U}})$ and $\overline{\mathbf{G}}=\mathbf{G}(\overline{\mathbf{U}})$. That is, the state $\overline{\mathbf{U}}$ should be a good physical state around which we can linearize in the subsonic case. Practical experience has shown that this is almost certainly guaranteed. (The positivity of density can indeed be satisfied if the multidimensional wave model satisfies an entropy 
condition, as shown in Balsara [4]. The positivity of the pressure is harder to guarantee.) If the state $\overline{\mathbf{U}}$ is not a good physical state, then one can resort to the numerical fluxes from a multidimensional Riemann solver without sub-structure (Balsara [5] [16], Balsara, Dumbser \& Abgrall [16], Vides, Nkonga \& Audit [67], Balsara \& Dumbser [17]). In practice, we have never found that to be necessary, but in the absence of a mathematical proof, it helps to make this statement for the sake of completeness.

Also notice that Balsara [16] has shown that sometimes the state in eqn. (6) can have very sharp linear variation. In some cases, the linear variation can exceed the values of the incoming states. In such situations, Balsara [16] recommends the use of a variant of the multidimensional limiter of Barth \& Frederickson [19]. Please see Appendix C of Balsara [16].

We also point out that it is only the inner, linearly degenerate, waves in the strongly interacting state that we would like to capture. For that reason, it helps to make the projection described in eqns. (26) and (27) of Balsara [16] in order to obtain the numerical fluxes.

In this section we have focused on the subsonic strongly-interacting state. The formulation in similarity variables also gives us useful perspective for the supersonic cases, please see (Balsara [16], Vides, Nkonga \& Audit [67], Balsara \& Dumbser [17]).

The formulation from Sub-section III.b is the easiest to implement and yields good results. The formulation from Sub-section III.c is not very suitable for numerical work because it does not couple variations in the fluxes to the variations in the state. We present it, nevertheless, for the sake of logical completeness. The formulation from Sub-section III.d is computationally quite costly because of the matrix inversions, though it may be useful to those who are interested in an analytical study of the multidimensional Riemann problem.

Eqns. (7c) and (8c) of this paper use a Zha-Bilgen flux vector splitting. Such flux-vector splittings have recently been analyzed by Toro and Vázquez-Cendón [65] within the context of one-dimensional Riemann solvers. Toro and Vázquez-Cendón find the original Zha-Bilgen splitting to be somewhat deficient. They, however, improve on the original Zha-Bilgen flux splitting by showing that if the pressure flux is built using the resolved state from the one dimensional Riemann solver then the Zha-Bilgen flux vector splitting works well. Our use of the resolved state in eqns. (7c) and (8c) of this paper is very much in the spirit of Toro and VázquezCendón which explains why the flux vector splitting works so well in this paper.

\section{IV) Accuracy Analysis}

In the next section we demonstrate the versatility of the MuSIC Riemann solver with least squares projection that we have presented in this paper. This section is devoted to accuracy analysis for Euler and MHD flow. The next section is devoted to several stringent tests drawn from hydrodynamics and MHD. Our results show our codes running with several different orders of accuracy. The MuSIC Riemann solver presented here can also accommodate structured and 
unstructured meshes. This is demonstrated by the fact that the Euler flow problems have been run with an unstructured mesh code while the MHD problems have been run with a divergencefree structured mesh code. Higher order spatial reconstruction is achieved by using the Weighted Essentially Non-Oscillatory (WENO) method (Jiang \& Shu [45], Balsara \& Shu [8], Abgrall [3], Dumbser \& Käser [31], Balsara et al. [12], Balsara et al. [14]). Higher order temporal accuracy is obtained by using ADER (Arbitrary DERivatives in space and time) time-evolution (Titarev \& Toro [59], [60] and Toro \& Titarev [61], Dumbser et al. [32], Balsara et al. [12], Balsara et al. [14]). Our treatment of MHD is divergence-free (Balsara \& Spicer [7], Balsara [9], [10], [11], Balsara \& Dumbser [18]). The GLM formulation of Dedner et al. [30] was not used in any of our simulations. Pressure positivity is enforced (Balsara [13]) to ensure that all states that are provided to the MuSIC Riemann solver have good positivity properties.

For all the problems described here, the multidimensional Riemann solver used the onedimensional HLLC Riemann solver as a building block. The Simpson rule was used within each face to evaluate a higher order numerical flux. In all cases, the problems were run twice, once with the corner fluxes based on the formulation described in Sub-section III.b and the second time with the formulation described in Sub-section III.d. Both formulations were found to work very well. The central point in the Simpson rule requires the use of a one-dimensional flux from a one-dimensional Riemann solver. For hydrodynamics, we used the flux vector splitting of Toro and Vázquez-Cendón [65]. For MHD, we used the one-dimensional HLLC Riemann solver from Li [47].

There is a slight difference in the temporal formulation in the structured and unstructured mesh codes. The structured mesh code makes only one call to the multidimensional Riemann solver per edge and per timestep. The unstructured mesh code uses higher order quadrature in time, with the result that the number of calls to the multidimensional Riemann problem per edge and per timestep is equal to the order of the code. Consequently, the unstructured mesh code has an, understandably, better timestep stability, permitting the use of a CFL (in two-dimensions) that is close to unity. The structured mesh code, despite fewer calls to the Riemann solver, achieves a CFL (in two-dimensions) of 0.8 at second and third order and 0.4 at fourth order. If a multidimensional Riemann solver is not used, both codes suffer a lower timestep stability; thus showing the beneficial role of the MuSIC Riemann solver in enabling larger timesteps.

In the next two sub-sections we show the accuracy of our codes with the MuSIC Riemann solver designed in this paper for Euler and MHD flows.

\section{IV.a) Accuracy Analysis for Hydrodynamical Vortex Problem}

In this hydrodynamical vortex problem, presented for example in Balsara \& Shu [8], an isentropic vortex propagates in form-preserving fashion at $45^{\circ}$ to the mesh. The computational domain has periodic boundaries and is given by $[-5,5] \times[-5,5]$. The vortex returns to its original location. An unstructured mesh was used for this problem. We do not describe it in detail here 
because it is well-known through the original citation. The exponential function in the velocity and temperature fluctuations for the vortex ensures that the fluctuations are quite close to zero at the domain boundaries. The stopping time was set to 10 time units in all cases. We report on the accuracy of the density after the vortex has completed one orbit in the computational domain in Table I. All the schemes meet their design accuracy.

TABLE I shows the accuracy analysis for the hydrodynamical vortex problem as measured in the density variable.

\begin{tabular}{|l|c|l|c|c|c|}
\hline Method & \# of zones & $L_{1}$ Error & $L_{1}$ Order & $L_{\infty}$ Error & $L_{\infty}$ Order \\
\hline $\begin{array}{l}\text { ADER-WENO } \\
2^{\text {nd }} \text { Order }\end{array}$ & & & & & \\
\hline & $\mathrm{h}=1 / 64$ & $1.7972 \mathrm{E}-01$ & & $2.1170 \mathrm{E}-02$ & \\
\hline & $\mathrm{h}=1 / 128$ & $4.6603 \mathrm{E}-02$ & 1.95 & $5.2465 \mathrm{E}-03$ & 2.01 \\
\hline & $\mathrm{h}=1 / 256$ & $1.1981 \mathrm{E}-02$ & 1.96 & $1.7058 \mathrm{E}-03$ & 1.62 \\
\hline & $\mathrm{h}=1 / 512$ & $3.0886 \mathrm{E}-03$ & 1.96 & $9.9955 \mathrm{E}-04$ & 0.77 \\
\hline $\begin{array}{l}\text { ADER-WENO } \\
3^{\text {rd }} \text { Order }\end{array}$ & & & & & \\
\hline & $\mathrm{h}=1 / 64$ & $4.4939 \mathrm{E}-02$ & & $7.4496 \mathrm{E}-03$ & \\
\hline & $\mathrm{h}=1 / 128$ & $5.7426 \mathrm{E}-03$ & 2.97 & $9.9640 \mathrm{E}-04$ & 2.90 \\
\hline & $\mathrm{h}=1 / 256$ & $7.0246 \mathrm{E}-04$ & 3.03 & $1.2577 \mathrm{E}-04$ & 2.99 \\
\hline & $\mathrm{h}=1 / 512$ & $8.8956 \mathrm{E}-05$ & 2.98 & $1.5956 \mathrm{E}-05$ & 2.98 \\
\hline $\begin{array}{l}\text { ADER-WENO } \\
4^{\text {th }} \text { Order }\end{array}$ & & & & & \\
\hline & $\mathrm{h}=1 / 64$ & $6.3998 \mathrm{E}-03$ & & & \\
\hline & $\mathrm{h}=1 / 128$ & $3.9278 \mathrm{E}-04$ & 4.03 & $6.8027 \mathrm{E}-05$ & 3.92 \\
\hline & $\mathrm{h}=1 / 256$ & $2.0689 \mathrm{E}-05$ & 4.25 & $4.4649 \mathrm{E}-06$ & 3.93 \\
\hline & $\mathrm{h}=1 / 512$ & $1.3332 \mathrm{E}-06$ & 3.96 & $2.6873 \mathrm{E}-07$ & 4.05 \\
\hline
\end{tabular}

\section{IV.b) Accuracy Analysis for MHD Vortex Problem}

The magnetized isodensity vortex problem described in Balsara [10] consists of a magnetized vortex moving in form-preserving fashion across a domain given by $[-5,5] \times[-5,5]$. Periodic boundaries are used for the domain. The magnetized vortex propagates at an angle of $45^{\circ}$ for a time of 10 units. A structured mesh was used for this problem. Since the problem is well-known in the literature, we do not describe it in detail here. We report on the accuracy of the x-magnetic field of the vortex after it has completed one orbit in the computational domain. All the schemes meet their design accuracy.

TABLE II shows the accuracy analysis for the MHD vortex problem as measured in the $x$ magnetic field.

\begin{tabular}{|l|l|l|l|l|l|}
\hline Method & \# of zones & $L_{1}$ Error & $L_{1}$ Order & $L_{\infty}$ Error & $L_{\infty}$ Order \\
\hline
\end{tabular}




\begin{tabular}{|l|c|c|c|c|c|}
\hline $\begin{array}{l}\text { ADER-WENO } \\
2^{\text {nd }} \text { Order }\end{array}$ & $64 \times 64$ & $3.2765 \mathrm{E}-03$ & & & \\
\hline & $128 \times 128$ & $8.0508 \mathrm{E}-04$ & 2.02 & $6.9540 \mathrm{E}-03$ & 1.90 \\
\hline & $256 \times 256$ & $2.0092 \mathrm{E}-04$ & 2.00 & $1.7523 \mathrm{E}-03$ & 1.98 \\
\hline & $512 \times 512$ & $5.1021 \mathrm{E}-05$ & 1.98 & $4.3802 \mathrm{E}-04$ & 2.00 \\
\hline & & & & & \\
\hline $\begin{array}{l}\text { ADER-WENO } \\
3^{\text {rd }} \text { Order }\end{array}$ & $64 \times 64$ & $5.3263 \mathrm{E}-04$ & & $7.9545 \mathrm{E}-03$ & \\
\hline & $128 \times 128$ & $6.8519 \mathrm{E}-05$ & 2.95 & $1.2964 \mathrm{E}-02$ & 2.61 \\
\hline & $256 \times 256$ & $8.8319 \mathrm{E}-06$ & 2.95 & $2.2338 \mathrm{E}-04$ & 2.53 \\
\hline & $512 \times 512$ & $1.1460 \mathrm{E}-06$ & 2.95 & $5.0752 \mathrm{E}-05$ & 2.14 \\
\hline & & & & & \\
\hline $\begin{array}{l}\text { ADER-WENO } \\
4^{\text {th }} \text { Order }\end{array}$ & $64 \times 64$ & $4.7886 \mathrm{E}-04$ & & $3.0588 \mathrm{E}-02$ & \\
\hline & $128 \times 128$ & $2.4074 \mathrm{E}-05$ & 4.31 & $1.4309 \mathrm{E}-03$ & 4.41 \\
\hline & $256 \times 256$ & $1.3433 \mathrm{E}-06$ & 4.16 & $6.2403 \mathrm{E}-05$ & 4.51 \\
\hline & $512 \times 512$ & $8.0985 \mathrm{E}-08$ & 4.05 & $3.7979 \mathrm{E}-06$ & 4.03 \\
\hline
\end{tabular}

\section{V) Test Problems}

\section{V.a) Hydrodynamical Test: Sod and Lax Problems on a Two-Dimensional Mesh}

The Sod and Lax problems are very well-known. We set the problems up on a two dimensional mesh and ran them using a third order ADER-WENO scheme with a CFL of 0.95. Fig. 6a shows the density from the Sod shock test problem along with the mesh structure, while Fig. $6 \mathrm{~b}$ plots the density in one dimension along with the exact solution of the Riemann problem. Fig. 6c shows the density from the Lax shock test problem along with the mesh structure, while Fig. 6d plots the density in one dimension along with the exact solution. All simulations in Fig. 6 show crisp shocks along with a well-resolved contact discontinuity that has a sharp profile. This demonstrates that retaining sub-structure in our MuSIC Riemann solver is very useful in resolving sub-structure in the flow.

\section{V.b) Hydrodynamical Test: Long Term Preservation of the Contact Discontinuity}

This problem was described in detail in Balsara \& Dumbser [17] so we do not repeat the description here. The problem consists of setting up a two-dimensional top hat density profile with no further variation in the pressure or velocities. The problem was run with a CFL of 0.95 to a final time of 10.0, which constitutes $\sim 3600$ time steps. A third order ADER-WENO scheme was used. Fig. 7a shows the density profile at the final time when the multidimensional Riemann solver with self-similar sub-structure from this paper is used. Fig. 7b shows the corresponding density profile as a one-dimensional plot. We see that the density profile has been crisply preserved. As with the previous test problem, this test problem underscores the value of retaining 
sub-structure in our MuSIC Riemann solver. It shows that the least squares projection described in this paper is a capable strategy for projecting the sub-structure in the flow.

\section{V.c) Hydrodynamical Test: Two-Dimensional Riemann Problems}

Since the advent of multidimensional Riemann solvers, the two-dimensional Riemann problems by Shuulz-Rinne et al. [58] have become very popular test problems. Both the multidimensional Riemann problems that we show here have been described in Balsara [4]. They have been run using the present multidimensional Riemann solver along with a third-order ADER-WENO scheme running with a CFL of 0.95 . An $h=1 / 1000$ unstructured mesh with $~ 2.3$ million elements was used. The stopping times for the first and second Riemann problems were 0.5 and 1.05 , respectively.

Figs. $8 \mathrm{a}$ and $8 \mathrm{~b}$ show the densities from the first and second multidimensional Riemann problems. Only the lower left portion of the computational domain is shown in Fig. 8b. We see that the roll-up of the Kelvin-Helmholtz instability is very crisply captured in both figures. It shows that the MuSIC Riemann solver can rapidly adjust to the changing strength and direction of the contact discontinuities on an unstructured mesh. The Riemann solver automatically adjusts to the changing flow conditions by introducing a time-evolving density gradient in the stronglyinteracting state that tracks the contact discontinuities and their time-evolution.

\section{V.d) Hydrodynamical Test: Double Mach Reflection Problem}

This test problem was first presented by Woodward and Colella [69] and, since the problem is very well-known, we do not repeat it here. Cockburn and Shu [26] carried out a resolution study using schemes of increasing order of accuracy. They found that they had to use at least a fourth order RKDG scheme at 1920x480 zone resolution to see the roll up of the vortex sheet.

In Fig. 9 we show the density variable from a simulation of the double Mach reflection problem which was run using a third order accurate ADER-WENO scheme. The MuSIC Riemann solver with least squares projection was used. An unstructured mesh with $\mathrm{h}=1 / 400$ and $\sim 1.4$ million elements was used. This resolution is roughly equivalent to that of Cockburn and Shu [26]. Fig. 9a shows entire density variable while Fig. 9b shows a zoom-in of the vortex sheet. We see that the vortex sheet roll-up is properly captured in our simulation despite our use of a third order scheme with vastly larger timesteps.

\section{V.e) MHD Test: Rotor Problem}

This well-known MHD problem was first documented in Balsara \& Spicer [7] and also Balsara [10], so we do not repeat the description here. The problem consists of a central, dense rotor in a uniformly magnetized static ambient medium. A uniform mesh of $1000 \times 1000$ zones was used. A third order ADER-WENO scheme was used. The MuSIC Riemann solver with least 
squares projection was used with an underlying one-dimensional HLLC Riemann solver. Fig. 10 shows the final result for the rotor problem, at a time of 0.25 . Figs. 10a, 10b, 10c and 10d show the density, pressure, Mach number and magnetic pressure at the final time. All the requisite MHD flow features are captured accurately in our simulations.

\section{V.f) MHD Test: Orszag Tang Problem}

This test problem suggested by Orszag \& Tang [50] is well-known and it is not described again here. It was initialized on a periodic domain spanning $[0 ; 2 \pi] \times[0,2 \pi]$ using a uniform mesh of $1000 \times 1000$ zones. It was run to a stopping time of $t=3.0$ with a third order accurate ADERWENO scheme. The MuSIC Riemann solver with least squares projection was used with an underlying one-dimensional HLLC Riemann solver. Figs. 11a, 11b, 11c and 11d show the density, pressure, Mach number and magnetic pressure at the final time. The simulation forms a current sheet with oppositely oriented x-components of magnetic field in the center of the computational domain, as can be surmised from Fig. 11d. The velocity field also shows fluid squirting out in the positive and negative $\mathrm{x}$-directions at the location of the current sheet.

\section{V.g) MHD Test: Field Loop Advection in Three-Dimensions}

This three-dimensional test problem was first presented in Gardiner \& Stone [39] and is based on an analogous two-dimensional problem in Gardiner \& Stone [38]. We do not repeat the details of the problem set up here. It consists of a weakly magnetized loop of magnetic field. In three-dimensions, the field loop is rotated so that the long axis of the loop points in the $(-\hat{\mathbf{x}}+2 \hat{\mathbf{z}}) / \sqrt{5}$ direction. The magnetic fields form loops around this axis and, in principle, the component of magnetic field along the axis of the loop should be zero. The problem is run with varying resolutions - i.e. with $32 \times 32 \times 64,64 \times 64 \times 128$ and $128 \times 128 \times 256$ zone meshes - on a periodic domain that spans $[-0.5,0.5] \times[-0.5,0.5] \times[-1,1]$ using a CFL of 0.45 . The loop is made to move along one of the diagonals of the mesh until it completes one orbit in one time unit. The problem was run on a structured mesh with a divergence-free third order accurate ADER-WENO scheme.

As the loop moves over the mesh, some of the magnetic energy decays as a function of time. Minimizing this magnetic energy decay is a measure of the quality of the solver. Likewise, errors in advection cause a small amount of magnetic field to develop along the axis of the loop. This results in the emergence of a small amount of magnetic energy in the component of magnetic field that is aligned with the axis. Minimizing the growth of energy in the on-axis magnetic field is again a measure of the quality of the numerical scheme. Both these magnetic energies are normalized with respect to the initial magnetic energy on the mesh. Fig. 12 shows the evolution of normalized magnetic energy for the field loop problem. Fig. 12a shows the magnetic energy as a function of time, where the magnetic energy is normalized by the initial magnetic energy. Fig. 12b shows the magnetic energy in the axial component of the magnetic 
field, which is also normalized by the initial magnetic energy. Results from simulations at three different resolutions are shown. We see that there is only a very small amount of decay in the normalized magnetic energy in Fig. 12a. Likewise, the build-up of magnetic energy in the axial direction is shown to be well-bounded in Fig. 12b. The results show the value of a higher order scheme operating with a multidimensional Riemann solver.

\section{V.h) MHD Test: Three Dimensional MHD Blast Wave Problem}

This problem was described in Section 5.2.3 of Balsara [5]. Here we simulate the same problem on a computational mesh of $128^{3}$ zones spanning $[-0.5,0.5]^{3}$. A very high pressure pulse is initially set up in the center of the domain. The resulting MHD blast wave travels through the plasma with a plasma- $\beta$ of 0.000513 . The simulation was run to a final time of 0.014 with a CFL of 0.45 and the multidimensional Riemann solver described here. A third order ADER-WENO scheme was used.

Figs. 13a through $13 \mathrm{~d}$ show the density, pressure, magnitude of the velocity and magnitude of the magnetic field respectively in the midplane of the three-dimensional MHD blast wave simulation. Despite the very low plasma- $\beta$, the pressure remains robustly positive and the flow variables have no spurious oscillations despite the use of a higher order WENO scheme. As the blast propagates, large velocities develop and the magnetic field is compressed so as to become even stronger than its initial value. The pressure is obtained by subtracting the kinetic and magnetic energies from the total energy density. The large values of the kinetic and magnetic energies can sometimes trigger a loss of pressure positivity in such simulations. This is not the case in Fig. 8b. We see, therefore, that along with the large time steps, the multidimensional Riemann solver-based method also does much better at maintaining pressure positivity. This stems from the ability to propagate magnetic fields very accurately in any needed direction on the computational mesh. Pressure-positivity preserving techniques from Balsara [13] also show their worth in this problem.

\section{VI) Conclusions}

This paper follows in the footsteps of the self-similar formulation of the multidimensional Riemann problem (Balsara [4], [5], [16], Balsara, Dumbser \& Abgrall [15], Balsara \& Dumbser [17]). The methods are predicated on the continuity of Lagrangian fluxes across the self-similarly moving boundary of the multidimensional wave model. Just as the quality of a one-dimensional approximate Riemann solver is improved by the inclusion of internal sub-structure, the quality of a multidimensional Riemann solver is also similarly improved and the first steps in that direction were taken in Balsara [5]. In Balsara [16], we developed, for the first time, a Galerkin projection to retrieve the sub-structure in the multidimensional Riemann problem. Explicitly enforcing shock jump conditions across a moving boundary is an alternative perspective on dealing with the moving, multidimensional wave model as developed in Vides, Nkonga and Audit [67]. At

some level, a shock jump is indeed an expression of the continuity of a Lagrangian flux across a 
moving boundary and that perspective lends a conceptual unity to those two formulations of the multidimensional Riemann solver. In this paper we show that the least squares projection strategy from Vides, Nkonga and Audit [67] can also be used advantageously to retrieve the substructure in the multidimensional Riemann problem. Both projection strategies, Galerkin projection and least squares projection, yield comparable results on a large range of test problems. Development of multiple viewpoints gives us the benefit of having multiple perspectives on the multidimensional Riemann problem.

A large number of test problems from Euler and divergence-free MHD are presented. Sub-sections III.b and III.d catalogue two alternative formulations for the MuSIC Riemann solver with least squares projection. We have tested both those formulations for Euler and MHD flow and found both of them to work very well. We show that schemes that are based on the multidimensional Riemann solver enable the use of a larger CFL number while retaining the desired order of accuracy. Several stringent divergence-free MHD problems that we present do not require any doubling of the dissipation when evaluating the electric field. The greater accuracy in advecting the magnetic field translates into an ability to treat MHD problems with lower values of plasma- $\beta$.

For more information on multidimensional Riemann solvers, please visit the following website: http://www.nd.edu/ dbalsara/Numerical-PDE-Course .

\section{Acknowledgements}

DSB acknowledges support via NSF grants NSF-AST-1009091, NSF-ACI-1307369 and NSF-DMS-1361197. DSB also acknowledges support via NASA grants from the Fermi program as well as NASA-NNX 12A088G. KFG acknowledges support via NSF-DMS-1361197 and \#245237 from the Simons Foundation. BN, EA, and JV acknowledge the financial support from the National French Research Program (ANR): ANEMOS (2011), ANR-11-MONU-002. MD was funded by the European Research Council (ERC) under the European Union's Seventh Framework Programme (FP7/2007-2013) with the research project STiMulUs, ERC Grant agreement no. 278267. The authors acknowledge PRACE for awarding access to the SuperMUC supercomputer of the Leibniz Rechenzentrum (LRZ) in Munich, Germany. Several simulations were performed on a cluster at UND that is run by the Center for Research Computing. Computer support on NSF's XSEDE computing resources is also acknowledged. 


\section{References}

[1] R.Abgrall, Approximation du problème de Riemann vraiment multidimensionnel des équations d'Euler par une méthode de type Roe, I: La linéarisation, C.R. Acad. Sci. Ser. I, 319 (1994) 499

[2] R.Abgrall, Approximation du problème de Riemann vraiment multidimensionnel des équations d'Euler par une méthode de type Roe, II: Solution du problème de Riemann approché, C.R. Acad. Sci. Ser. I, 319 (1994) 625

[3] R. Abgrall, On essentially non-oscillatory schemes on unstructured meshes : Analysis and implementation, J. Comput. Phys., 114 (1994) 45-58

[4] D.S. Balsara, Multidimensional HLLE Riemann solver; Application to Euler and Magnetohydrodynamic Flows, J. Comput. Phys., 229 (2010) 1970-1993

[5] D.S. Balsara, A two-dimensional HLLC Riemann solver for conservation laws: Application to Euler and magnetohydrodynamic flows, Journal of Computational Physics 231 (2012) 74767503

[6] D.S. Balsara, Linearized formulation of the Riemann problem for adiabatic and isothermal magnetohydrodynamics, Astrophysical Journal Supplement 116 (1998) 119

[7] D.S. Balsara and D.S. Spicer, A staggered mesh algorithm using high order Godunov fluxes to ensure solenoidal magnetic fields in magnetohydrodynamic simulations, Journal of Computational Physics 149 (1999) 270-292

[8] D.S. Balsara and C.-W. Shu, Monotonicity preserving weighted non-oscillatory schemes with increasingly high order of accuracy, Journal of Computational Physics, 160 (2000) 405-452

[9] D.S. Balsara, Divergence-free adaptive mesh refinement for magnetohydrodynamics, Journal of Computational Physics 174 (2001) 614-648

[10] D. S. Balsara, Second-order-accurate schemes for magnetohydrodynamics with divergencefree reconstruction, Astrophysical Journal Supplement 151 (2004) 149-184

[11] D.S. Balsara, Divergence-free reconstruction of magnetic fields and WENO schemes for magnetohydrodynamics, J. Comput. Phys., 228 (2009) 5040-5056

[12] Balsara, D.S., Rumpf, T., Dumbser, M. \& Munz, C.-D., Efficient, high-accuracy ADERWENO schemes for hydrodynamics and divergence-free magnetohydrodynamics, Journal of Computational Physics, 228 (2009) 2480 
[13] Balsara, D.S., Self-Adjusting, Positivity Preserving High Order Schemes for Hydrodynamics and Magnetohydrodynamics, Journal of Computational Physics, 231 (2012) 7504-7517

[14] Balsara, D.S., Dumbser, M., Meyer, C., Du, H. \& Xu, Z., Efficient Implementation of ADER schemes for Euler and Magnetohydrodynamic flow on structured meshes - Comparison with Runge-Kutta methods, Journal of Computational Physics, 235 (2013) 934-969

[15] D.S. Balsara, M. Dumbser and R. Abgrall, Multidimensional HLL and HLLC Riemann Solvers for Unstructured Meshes - With Application to Euler and MHD Flows, Journal of Computational Physics 261 (2014) 172-208

[16] D.S. Balsara, Multidimensional Riemann Problem with Self-Similar Internal Structure Part I - Application to Hyperbolic Conservation Laws on Structured Meshes, Journal of Computational Physics 277 (2014) 163-200

[17] D.S. Balsara and M. Dumbser, Multidimensional Riemann Problem with Self-Similar Internal Structure - Part II - Application to Hyperbolic Conservation Laws on Unstructured Meshes, to appear, Journal of Computational Physics (2015)

[18] D.S. Balsara and M. Dumbser, Divergence-Free MHD on Unstructured Meshes Using High Order Finite Volume Schemes Based on Multidimensional Riemann Solvers, submitted, Journal of Computational Physics (2015)

[19] T.J. Barth and P.O. Frederickson, Higher order solution of the Euler equations on unstructured grids using quadratic reconstruction, AIAA Paper no. 90-0013, 28 ${ }^{\text {th }}$ Aerospace Sciences Meeting, January (1990)

[20] P. Batten, N. Clarke, C. Lambert and D.M. Causon, On the choice of wavespeeds for the HLLC Riemann solver, SIAM J. Sci. Comput., 18, (1997) 1553-1570

[21] S.J. Billett and E.F. Toro, On WAF-type schemes for multidimensional hyperbolic conservation laws, Journal of Computational Physics, 130 (1997) 1-24

[22] M.Brio, A.R. Zakharian and G.M. Webb, Two-dimensional Riemann solver for Euler equations of gas dynamics, J. Comput. Phys., 167 (2001) 177-195

[23] P. Cargo \& G. Gallice, Roe matrices for ideal MHD and systematic construction of Roe matrices for systems of conservation laws, J. Comput. Phys., 136 (1997) 446

[24] A. Chakraborty and E.F. Toro. Development of an approximate Riemann solver for the steady supersonic Euler equations. The Aeronautical Journal. Vol. 98, pages 325-339, 1994.

[25] A.J. Chorin, Random choice solutions of hyperbolic systems, J. Comput. Phys., 22 (1976) 517 
[26] B. Cockburn and C.-W. Shu, The Runge-Kutta discontinuous Galerkin method for Conservation Laws V, Journal of Computational Physics 141 (1998) 199-224

[27] P. Colella, A direct Eulerian MUSCL scheme for gas dynamics, SIAM, J. Sci. Statist. Comput., 6 (1985) 104

[28] P. Colella, Multidimensional Upwind methods for hyperbolic conservation laws, J. Comput. Phys., 87, (1990) 171

[29] P. Colella and P.R. Woodward, The piecewise parabolic method (PPM) for gas-dynamical simulations, Journal of Computational Physics 54 (1984) 174-201

[30] A. Dedner, F. Kemm, D. Kröener, C.-D. Munz, T. Schnitzer, M. Wesenberg, Hyperbolic divergence cleaning for MHD equations, Journal of Computational Physics 175 (2002) 645-673

[31] M., Dumbser, M., Käser, Arbitary high order non-oscillatory finite volume schemes on unstructured meshes for linear hyperbolic systems, Journal of Computational Physics, 221 (2007) 693-723

[32] Dumbser, M., Balsara, D.S., Toro, E.F., Munz, C.-D., A unified framework for the construction of one-step finite volume and discontinuous Galerkin schemes on unstructured meshes, Journal of Computational Physics, 227 (2008) 8209-8253

[33] M. Dumbser and E. F. Toro. A simple extension of the Osher Riemann solver to nonconservative hyperbolic systems, Journal of Scientific Computing, 48:70 (2011)88

[34] B.Einfeldt, On Godunov-type methods for gas dynamics, SIAM J. Numer. Anal., 25(3) (1988) 294-318

[35] B.Einfeldt, C.-D. Munz, P.L. Roe \& B. Sjogreen, On Godunov-type methods near low densities, J. Comput. Phys., 92 (1991) 273-295

[36] M. Fey, Multidimensional upwinding 1. The method of transport for solving the Euler equations, J. Comput. Phys., 143 (1998) 159

[37] M. Fey, Multidimensional upwinding 2. Decomposition of the Euler equation into advection equation, J. Comput. Phys., 143 (1998) 181

[38] T. Gardiner \& J.M. Stone, An unsplit Godunov method for ideal MHD via constrained transport, Journal of Computational Physics, 205 (2005), 509

[39] T. Gardiner \& J.M. Stone, An unsplit Godunov method for ideal MHD via constrained transport in three dimensions, Journal of Computational Physics, 227 (2008), 4123

[40] H. Gilquin, J. Laurens, and C. Rosier, Multidimensional Riemann problems for linear hyperbolic systems, Notes Numer. Fluid Mech., 43 (1993) 284 
[41] S.K. Godunov, Finite Difference Methods for the Computation of Discontinuous Solutions of the Equations of Fluid Dynamics, Mathematics of the USSR, Sbornik. 47 (1959) 271-306

[42] S.K. Godunov, Numerical Solution of Multi-dimensional Problems in Gas Dynamics, Nauka Press, Moscow (1976)

[43] K.F. Gurski, An HLLC-type approximate Riemann solver for ideal magnetohydrodynamics, SIAM J. Sci. Comput. 25 (2004) 2165

[44] A. Harten, P.D. Lax and B. van Leer, On upstream differencing and Godunov-type schemes for hyperbolic conservation laws, SIAM Rev. (1983) 25, 289-315

[45] Jiang, G.-S. and Shu, C.-W., Efficient implementation of weighted ENO schemes, Journal of Computational Physics, 126 (1996) 202-228

[46] R.J. LeVeque, Wave propagation algorithms for multidimensional hyperbolic systems, J. Comput. Phys. 131 (1997) 327

[47] S.-T. Li, An HLLC Riemann solver for magnetohydrodynamics, J. Comput. Phys., 203 (2005) 344

[48] M. Lukacsova-Medvidova, K.W. Morton, G. Warnecke, Finite volume evolution Galerkin methods for Euler equations of gas dynamics, Int. J. Num. Methods in Fluids, 40 (2002) 425

[49] T. Miyoshi and K. Kusano, A multi-state HLL approximate Riemann solver for ideal magnetohydrodynamics, J. Comput. Phys., 208 (2005) 315-344

[50] S. A. Orszag and C. M. Tang, Small-scale structure of two-dimensional magnetohydrodynamic turbulence, Journal of Fluid Mechanics, 90 (1979) 129

[51] S. Osher and F. Solomon, Upwind Difference Schemes for Hyperbolic Systems of Conservation Laws, Mathematics of Computation, 38(158) (1982) 339

[52] P.L. Roe, Approximate Riemann solver, parameter vectors and difference schemes, Journal of Computational Physics 43 (1981) 357-372

[53] P.L. Roe, Discrete models for the numerical analysis of time-dependent multidimensional gas dynamics, J. Comput. Phys., 63 (1986) 458

[54] P. L. Roe and D. S. Balsara, Notes on the eigensystem of magnetohydrodynamics, SIAM Journal of applied Mathematics 56 (1996), 57

[55] C.B. Rumsey, B. van Leer \& P.L. Roe, A multidimensional flux function with application to the Euler and Navier-Stokes equations, J. Comput. Phys., 105 (1993) 306 
[56] V.V. Rusanov, Calculation of interaction of non-steady shock waves with obstacles, J. Comput. Math. Phys. USSR, 1 (1961) 267

[57] J. Saltzman, An Unsplit 3D Upwind Method for Hyperbolic Conservation Laws, J. Comput. Phys., 115 (1994) 153

[58] C.W. Schulz-Rinne, J.P. Collins, and H.M. Glaz, Numerical solution of the Riemann problem for two-dimensional gas dynamics, SIAM J. Sci. Comput., 14(7) (1993) 1394-1414

[59] Titarev, V.A. and Toro, E.F., ADER: arbitrary high order Godunov approach, Journal of Scientific Computing 17 (1-4) (2002) 609-618

[60] Titarev, V.A. and Toro, E.F., ADER schemes for three-dimensional nonlinear hyperbolic systems, Journal of Computational Physics, 204 (2005) 715-736

[61] Toro, E.F. and Titarev, V.A., Solution of the generalized Riemann problem for advection reaction equations, Proceedings of the Royal Society of London, Series A 458 (2002) 271-281

[62] E.F. Toro, M. Spruce and W. Speares, Restoration of contact surface in the HLL Riemann solver, Shock Waves, 4 (1994) 25-34

[63] E F Toro, M Spruce and W Speares. Restoration of the contact surface in the Harten-Laxvan Leer Riemann solver. Shock Waves. Vol. 4 (1994) pages 25-34

[64] E F Toro, M Spruce and W Speares, Restoration of the contact surface in the HLL Riemann solver, Technical report CoA 9204. Department of Aerospace Science, College of Aeronautics, Cranfield Institute of Technology. UK. June, 1992

[65] Toro and Vázquez-Cendón, Flux splitting schemes for the Euler equations, Computers \& Fluids, 70 (2012) 1-12

[66] B. van Leer, Toward the Ultimate Conservative Difference Scheme. V. A Second-Order Sequel to Godunov's Method, J. Comput. Phys., 32 (1979) 101

[67] J. Vides, B. Nkonga \& E. Audit, A simple two-dimensional extension of the HLL Riemann solver for hyperbolic conservation laws, J. Comput. Phys., 280(1) (2015) 643-675

[68] B. Wendroff, A two-dimensional HLLE Riemann solver and associated Godunov-type difference scheme for gas dynamics, Computers and Mathematics with Applications, 38 (1999) 175-185

[69] P. Woodward and P. Colella, The numerical simulation of two-dimensional fluid flow with strong shocks, Journal of Computational Physics 54 (1984), 115-173

[70] Z.C. Zha and E. Bilgen, Numerical solutions of Euler equations by using a new flux vector splitting scheme, International Journal for Numerical Methods in Fluids, 17 (1993) 115-144 


\section{Appendix A}

Consider that a 1D HLLC Riemann solver is invoked on each face of our multidimensional wave model. We then have two segments within each flat wavefront where the shock jump conditions are imposed. By construction, the resulting model is a convex polygon with " $I_{\max }$ " faces (see Section II). In such a situation, equations (16) and (17), along with their analogues in each of the faces of the polygon, have the structure

$$
\mathbf{M}_{2 I_{\max } \times 2} \mathbf{V}=\mathbf{b}, \quad \text { with } \quad \mathbf{V}=\left(\mathbf{U}_{\xi}, \mathbf{U}_{\psi}\right)^{T} .
$$

Note that the matrix $\mathbf{M}$ then has dimension $2 I_{\max } \times 2$ and its explicit structure can be written as

$$
\mathbf{M}_{2 I_{\max } \times 2}=\left(\begin{array}{cc}
l_{P 1 C 1} \xi_{P 1 C 1} & l_{P 1 C 1} \psi_{P 1 C 1} \\
l_{C 1 P 2} \xi_{C 1 P 2} & l_{C 1 P 2} \psi_{C 1 P 2} \\
l_{P 2 C 2} \xi_{P 2 C 2} & l_{P 2 C 2} \psi_{P 2 C 2} \\
l_{C 2 P 3} \xi_{C 2 P 3} & l_{C 2 P 3} \psi_{C 2 P 3} \\
\vdots & \vdots \\
l_{P I_{\max } C I_{\max }} \xi_{P I_{\max } C I_{\max }} & l_{P I_{\max } C I_{\max }} \psi_{P I_{\max } C I_{\max }} \\
l_{C I_{\max } P I} \xi_{C I_{\max } P 1} & l_{C I_{\max } P I} \psi_{C I_{\max } P 1}
\end{array}\right)=\left(\begin{array}{cc}
\alpha_{1} & \beta_{1} \\
\gamma_{1} & \delta_{1} \\
\alpha_{2} & \beta_{2} \\
\gamma_{2} & \delta_{2} \\
\vdots & \vdots \\
\alpha_{I_{\max }} & \beta_{I_{\max }} \\
\gamma_{I_{\max }} & \delta_{I_{\max }}
\end{array}\right),
$$

having defined the variables $\alpha_{i}=l_{P i C i} \xi_{P i C i}, \beta_{i}=l_{P i C i} \psi_{P i C i}, \gamma_{i}=l_{C i P i+1} \xi_{C i P i+1}$ and $\delta_{i}=l_{C i P i+1} \psi_{C i P i+1}$. The structure of vector $\mathbf{b}$ can be obtained directly from the right-hand side of equations (16), (17), and their corresponding analogues.

It is clear that we have an overdetermined set of linear equations. The linear least squares approach relies on approximately solving (A.1) by finding the least squares solution $\mathbf{V}$ that minimizes $\|r\|$, where $r=\mathbf{M}_{2 I_{\max } \times 2} \mathbf{V}-\mathbf{b}$ is the residual or error. To find $\mathbf{V}$, we then minimize the squared norm

$\|r\|^{2}=\langle\mathbf{M V}-\mathbf{b}, \mathbf{M V}-\mathbf{b}\rangle=\mathbf{V}^{T} \mathbf{M}^{T} \mathbf{M V}-2 \mathbf{b}^{T} \mathbf{M V}+\mathbf{b}^{T} \mathbf{b}$

where the transpose of an element $(\cdot)$ is denoted $(\cdot)^{T}$. The minimum is then obtained by setting the gradient equal to zero, i.e.,

$\nabla_{\mathbf{V}}\|r\|^{2}=2 \mathbf{M}^{T} \mathbf{M V}-2 \mathbf{M}^{T} \mathbf{b}=0$

which yields the normal equations in matrix notation 


\section{$\mathbf{M}^{T} \mathbf{M V}=\mathbf{M}^{T} \mathbf{b}$.}

The above system gives an approximate solution for $\mathbf{U}_{\xi}$ and $\mathbf{U}_{\psi}$ provided the symmetric matrix $\mathbf{L}=\mathbf{M}^{T} \mathbf{M}$ is invertible. Here, the matrix $\mathbf{L}$ can be written as follows

$\mathbf{L}=\left(\begin{array}{lc}\sum_{i=1}^{I_{\max }}\left(\alpha_{i}^{2}+\gamma_{i}^{2}\right) & \sum_{i=1}^{I_{\max }}\left(\alpha_{i} \beta_{i}+\gamma_{i} \delta_{i}\right) \\ \sum_{i=1}^{I_{\max }}\left(\alpha_{i} \beta_{i}+\gamma_{i} \delta_{i}\right) & \sum_{i=1}^{I_{\max }}\left(\beta_{i}^{2}+\delta_{i}^{2}\right)\end{array}\right)$.

The purpose of Appendix B is to prove that this matrix is indeed invertible so that $\mathbf{V}=\mathbf{L}^{-1} \mathbf{M}^{T} \mathbf{b}$

\section{Appendix B}

A direct approach to prove that $\mathbf{L}$ (A.2) is invertible relies on showing that its determinant $\operatorname{det}(\mathbf{L})$ is non-zero. Although this proof is longer than the alternative one we will provide afterwards, it yields important information for later interpretation. As the determinant of a $2 \times 2$ matrix is straightforward, we simply write

$\operatorname{det}(\mathbf{L})=\left(\alpha_{i} \alpha_{i}+\gamma_{i} \gamma_{i}\right)\left(\beta_{j} \beta_{j}+\delta_{j} \delta_{j}\right)-\left(\alpha_{i} \beta_{i}+\gamma_{i} \delta_{i}\right)\left(\alpha_{j} \beta_{j}+\gamma_{j} \delta_{j}\right)$

having used the Einstein summation convention where repeated indices are summed over. This is equivalent to writing

$$
\operatorname{det}(\mathbf{L})=\frac{1}{2}\left[\left(\alpha_{i} \alpha_{i}+\gamma_{i} \gamma_{i}\right)\left(\beta_{j} \beta_{j}+\delta_{j} \delta_{j}\right)+\left(\alpha_{j} \alpha_{j}+\gamma_{j} \gamma_{j}\right)\left(\beta_{i} \beta_{i}+\delta_{i} \delta_{i}\right)-2\left(\alpha_{i} \beta_{i}+\gamma_{i} \delta_{i}\right)\left(\alpha_{j} \beta_{j}+\gamma_{j} \delta_{j}\right)\right] .
$$

Thus, by regrouping, we obtain

$$
\operatorname{det}(\mathbf{L})=\frac{1}{2}\left[\left(\alpha_{i} \beta_{j}-\alpha_{j} \beta_{i}\right)^{2}+\left(\alpha_{i} \delta_{j}-\beta_{i} \gamma_{j}\right)^{2}+\left(\gamma_{i} \beta_{j}-\delta_{i} \alpha_{j}\right)^{2}+\left(\gamma_{i} \delta_{j}-\delta_{i} \gamma_{j}\right)^{2}\right]
$$

Notice that the determinant has been expressed as a sum of squares. It, therefore, suffices to prove that any one of them is non-zero so that $\operatorname{det}(\mathbf{L})>0$. For this, focus on the second term and take $i=1$ and $j=1$, i.e., focus on the square binomial $\left(\alpha_{1} \delta_{1}-\beta_{1} \gamma_{1}\right)^{2}$. Employing the cross product notation $\mathbf{r} \times \mathbf{S}=r_{x} s_{y}-r_{y} s_{x}$ and recalling that $\alpha_{1}=l_{P 1 C 1} \xi_{P 1 C 1}, \quad \beta_{1}=l_{P 1 C 1} \psi_{P 1 C 1}$, $\gamma_{1}=l_{C 1 P 2} \xi_{C 1 P 2}$ and $\delta_{1}=l_{C 1 P 2} \psi_{C 1 P 2}$, we write

$$
\left(\alpha_{1} \delta_{1}-\beta_{1} \gamma_{1}\right)^{2}=\left(l_{P 1 C 1} l_{C 1 P 2}\right)^{2}\left[\left(\xi_{P 1 C 1}, \psi_{P 1 C 1}\right) \times\left(\xi_{C 1 P 2}, \psi_{C 1 P 2}\right)\right]^{2}
$$


Due to the convexity of the multidimensional wave model, it is clear that this term is non-zero because: 1$)$ the points $\left(\xi_{P 1 C 1}, \psi_{P 1 C 1}\right)$ and $\left(\xi_{C 1 P 2}, \psi_{C 1 P 2}\right)$ are measured relative to the centroid $\kappa$ so they are non-zero vectors; 2) those same points are mutually disjoint and lie on the same finitesized wavefront; 3) the lengths of the sub-segments $P_{1} C_{1}$ and $C_{1} P_{2}$ are positive quantities. Consequently, each cross product of the type $\left(l_{P i C i+1} l_{C i P i+1}\right)^{2}\left(\xi_{P i C i}, \psi_{P i C i}\right) \times\left(\xi_{C i P i+1}, \psi_{C i P i+1}\right)$ is surely non-zero. Thus, $\operatorname{det}(\mathbf{L})>0$.

\section{General Case:}

In general, if the 1D Riemann solver invoked at each face of our wave model has $W \geq 2$ waves, the matrix $\mathbf{M}$ associated with equations (16) and (17) will take the form

$$
\mathbf{M}_{W I_{\max } \times 2}=\left(\begin{array}{cc}
\bar{\xi}_{1} & \overline{\boldsymbol{\Psi}}_{1} \\
\bar{\xi}_{2} & \overline{\boldsymbol{\Psi}}_{2} \\
\vdots & \vdots \\
\bar{\xi}_{I_{\max }} & \overline{\boldsymbol{\Psi}}_{I_{\max }}
\end{array}\right),
$$

having defined, for each $i \in\left\{1, \ldots, I_{\max }\right\}$,

$$
\bar{\xi}_{i}=\left(l_{i}^{1} \xi_{i}^{1}, l_{i}^{2} \xi_{i}^{2}, \ldots, l_{i}^{W-1} \xi_{i}^{W-1}\right)^{T} \quad \text { and } \quad \bar{\psi}_{i}=\left(l_{i}^{1} \psi_{i}^{1}, l_{i}^{2} \psi_{i}^{2}, \ldots, l_{i}^{W-1} \psi_{i}^{W-1}\right)^{T}
$$

Let $l_{i}^{j}$ and $\left(\xi_{i}^{j}, \psi_{i}^{j}\right)$ be respectively the length and midpoint of the segment that corresponds to the $j^{\text {th }}$ constant state in the $i^{\text {th }}$ wavefront. One jump relation is imposed at each of the midpoints. The symmetric matrix $\mathbf{L}=\left(\mathbf{M}_{W I_{\max } \times 2}\right)^{T} \mathbf{M}_{W I_{\max } \times 2}$ then takes the form

$$
\mathbf{L}=\left(\begin{array}{ll}
\sum_{i=1}^{I_{\max }} \sum_{j=1}^{W-1}\left(l_{i}^{j}\right)^{2}\left(\xi_{i}^{j}\right)^{2} & \sum_{i=1}^{I_{\max }} \sum_{j=1}^{W-1}\left(l_{i}^{j}\right)^{2} \xi_{i}^{j} \psi_{i}^{j} \\
\sum_{i=1}^{I_{\max }} \sum_{j=1}^{W-1}\left(l_{i}^{j}\right)^{2} \xi_{i}^{j} \psi_{i}^{j} & \sum_{i=1}^{I_{\max }} \sum_{j=1}^{W-1}\left(l_{i}^{j}\right)^{2}\left(\psi_{i}^{j}\right)^{2}
\end{array}\right) .
$$

\section{Theorem I}

Let $\left(\xi_{i}^{j}, \psi_{i}^{j}\right)$ and $l_{i}^{j}$ be the midpoint and length of the $j^{\text {th }}$ segment formed at the wavefront $i$ of a multidimensional wave model constructed by employing W-waved $1 D$ Riemann problems; $i=\left\{1, \ldots, I_{\max }\right\}, j=\{1, \ldots, W-1\}$. If the model's polygon satisfies convexity and all points are measured relative to its centroid $\kappa$, then the least squares matrix (B.1) is invertible. 


\section{Proof}

To prove that $\mathbf{L}$ can be inverted, we first will show that it is positive definite, i.e., show that $\mathbf{z}^{T} \mathbf{L z}>0$ for every non-zero column vector $\mathbf{z}=\left(z_{1}, z_{2}\right)$. We then have

$$
\begin{aligned}
\mathbf{z}^{T} \mathbf{L} \mathbf{z} & =\sum_{i=1}^{I_{\max }} \sum_{j=1}^{W-1}\left(l_{i}^{j}\right)^{2}\left(z_{1} \xi_{i}^{j}\right)^{2}+2 \sum_{i=1}^{I_{\max }} \sum_{j=1}^{W-1}\left(l_{i}^{j}\right)^{2}\left(z_{1} \xi_{i}^{j}\right)\left(z_{2} \psi_{i}^{j}\right)+\sum_{i=1}^{I_{\max }} \sum_{j=1}^{W-1}\left(l_{i}^{j}\right)^{2}\left(z_{2} \psi_{i}^{j}\right)^{2} \\
& =\sum_{i=1}^{I_{\max }} \sum_{j=1}^{W-1}\left(l_{i}^{j}\right)^{2}\left(z_{1} \xi_{i}^{j}+z_{2} \psi_{i}^{j}\right)^{2}
\end{aligned}
$$

The points are $\left(\xi_{i}^{j}, \psi_{i}^{j}\right)$ are non-zero vectors since they are measured relative to $\kappa$; in total, we have $I_{\max }(W-1)$ non-zero points. Given that the wave model is convex, we are certain that each side of the polygon contains at least one segment with a positive length $l_{i}^{j}$ and unique midpoint $\left(\xi_{i}^{j}, \psi_{i}^{j}\right)$. Therefore, at least $I_{\max }$ of the points are distinct and $\mathbf{z}^{T} \mathbf{L z}=0$ only if $\mathbf{z}=\mathbf{0}$. Hence, $\mathbf{z}^{T} \mathbf{L z}>0$ and since every positive definite matrix is invertible, $\mathbf{L}$ is invertible.

\section{Appendix C}

Using an approach similar to the one employed in Appendix A, we consider equations (20) and (21), with their analogues in each of the polygon faces, and write

$$
\mathbf{M}_{2 I_{\max } \times 6} \mathbf{V}=\mathbf{b}
$$

with $\mathbf{V}=\left(\mathbf{U}_{\xi}, \mathbf{U}_{\psi}, \mathbf{F}_{\xi}, \mathbf{F}_{\psi}, \mathbf{G}_{\xi}, \mathbf{G}_{\psi}\right)^{T}$ and the vector $\mathbf{b}$ denoting the right-hand side of the equations. We have assumed once more that the 1D Riemann solver invoked at each face of the wave model is of HLLC type and this is why the matrix $\mathbf{M}$ has dimension $2 I_{\max } \times 6$. Specifically, we have 
$\mathbf{M}=\left(\begin{array}{cccccc}\eta_{1 x} \alpha_{1} & \eta_{1 x} \beta_{1} & \eta_{1 y} \alpha_{1} & \eta_{1 y} \beta_{1} & -S_{1} \alpha_{1} & -S_{1} \beta_{1} \\ \eta_{1 x} \gamma_{1} & \eta_{1 x} \delta_{1} & \eta_{1 y} \gamma_{1} & \eta_{1 y} \delta_{1} & -S_{1} \gamma_{1} & -S_{1} \delta_{1} \\ \eta_{2 x} \alpha_{2} & \eta_{2 x} \beta_{2} & \eta_{2 y} \alpha_{2} & \eta_{2 y} \beta_{2} & -S_{2} \alpha_{2} & -S_{2} \beta_{2} \\ \eta_{2 x} \gamma_{2} & \eta_{2 x} \delta_{2} & \eta_{2 y} \gamma_{2} & \eta_{2 y} \delta_{2} & -S_{2} \gamma_{2} & -S_{2} \delta_{2} \\ \vdots & \vdots & \vdots & \vdots & \vdots & \vdots \\ \eta_{I_{\max } x} \alpha_{I_{\max }} & \eta_{I_{\max } x} \beta_{I_{\max }} & \eta_{I_{\max } y} \alpha_{I_{\max }} & \eta_{I_{\max } y} \beta_{I_{\max }} & -S_{I_{\max }} \alpha_{I_{\max }} & -S_{I_{\max }} \beta_{I_{\max }} \\ \eta_{I_{\max } x} \gamma_{I_{\max }} & \eta_{I_{\max } x} \delta_{I_{\max }} & \eta_{I_{\max } y} \gamma_{I_{\max }} & \eta_{I_{\max } y} \delta_{I_{\max }} & -S_{I_{\max }} \gamma_{I_{\max }} & -S_{I_{\max }} \delta_{I_{\max }}\end{array}\right)$,

after having set $\alpha_{i}=l_{P i C i} \xi_{P i C i}, \beta_{i}=l_{P i C i} \psi_{P i C i}, \gamma_{i}=l_{C i P i+1} \xi_{C i P i+1}$ and $\delta_{i}=l_{C i P i+1} \psi_{C i P i+1}$. Now, we want to show that the least squares matrix $\mathbf{L}=\mathbf{M}^{T} \mathbf{M}$ is always invertible. Let us first define $a_{i}=\alpha_{i}^{2}+\gamma_{i}^{2} \quad ; \quad b_{i}=\alpha_{i} \beta_{i}+\gamma_{i} \delta_{i} \quad ; \quad c_{i}=\beta_{i}^{2}+\delta_{i}^{2}$

Using these variables, we are able to write the symmetric matrix $\mathbf{L}$ as

$$
\mathbf{L}=\left(\begin{array}{cc:cc:cc}
\sum_{i} a_{i} \eta_{i x}^{2} & \sum_{i} b_{i} \eta_{i x}^{2} & \sum_{i} a_{i} \eta_{i x} \eta_{i y} & \sum_{i} b_{i} \eta_{i x} \eta_{i y} & -\sum_{i} a_{i} S_{i} \eta_{i x} & -\sum_{i} b_{i} S_{i} \eta_{i x} \\
\sum_{i} b_{i} \eta_{i x}^{2} & \sum_{i} c_{i} \eta_{i x}^{2} & \sum_{i} b_{i} \eta_{i x} \eta_{i y} & \sum_{i} c_{i} \eta_{i x} \eta_{i y} & -\sum_{i} b_{i} S_{i} \eta_{i x} & -\sum_{i} c_{i} S_{i} \eta_{i x} \\
\sum_{i} a_{i} \eta_{i x} \eta_{i y} & \sum_{i} b_{i} \eta_{i x} \eta_{i y} & \sum_{i} a_{i} \eta_{i y}^{2} & \sum_{i} b_{i} \eta_{i y}^{2} & -\sum_{i} a_{i} S_{i} \eta_{i y} & -\sum_{i} b_{i} S_{i} \eta_{i y} \\
\sum_{i} b_{i} \eta_{i x} \eta_{i y} & \sum_{i} c_{i} \eta_{i x} \eta_{i y} & \sum_{i} b_{i} \eta_{i y}^{2} & \sum_{i} c_{i} \eta_{i y}^{2} & -\sum_{i} b_{i} S_{i} \eta_{i y} & -\sum_{i} c_{i} S_{i} \eta_{i y} \\
\hdashline-\sum_{i} a_{i} S_{i} \eta_{i x} & -\sum_{i} b_{i} S_{i} \eta_{i x} & -\sum_{i} a_{i} S_{i} \eta_{i y} & -\sum_{i} b_{i} S_{i} \eta_{i y} & \sum_{i} a_{i} S_{i}^{2} & \sum_{i} b_{i} S_{i}^{2} \\
-\sum_{i} b_{i} S_{i} \eta_{i x} & -\sum_{i} c_{i} S_{i} \eta_{i x} & -\sum_{i} b_{i} S_{i} \eta_{i y} & -\sum_{i} c_{i} S_{i} \eta_{i y} & \sum_{i} b_{i} S_{i}^{2} & \sum_{i} c_{i} S_{i}^{2}
\end{array}\right) .
$$

To prove that $\mathbf{L}$ is invertible, we choose to show that it is positive definite, i.e., $\mathbf{z}^{T} \mathbf{L z}>0$ for all non-zero column vectors $\mathbf{z}$ in $\mathbb{R}^{6}$. After some algebraic manipulations, we find

$$
\begin{aligned}
\mathbf{z}^{T} \mathbf{L z}= & \sum_{i}\left[\alpha_{i}\left(\left(\eta_{i x}, \eta_{i y}\right) \cdot\left(z_{1}, z_{3}\right)-S_{i} z_{5}\right)+\beta_{i}\left(\left(\eta_{i x}, \eta_{i y}\right) \cdot\left(z_{2}, z_{4}\right)-S_{i} z_{6}\right)\right]^{2} \\
& +\sum_{i}\left[\gamma_{i}\left(\left(\eta_{i x}, \eta_{i y}\right) \cdot\left(z_{1}, z_{3}\right)-S_{i} z_{5}\right)+\delta_{i}\left(\left(\eta_{i x}, \eta_{i y}\right) \cdot\left(z_{2}, z_{4}\right)-S_{i} z_{6}\right)\right]^{2} .
\end{aligned}
$$

Given that $\left(\alpha_{i}, \beta_{i}\right)$ and $\left(\gamma_{i}, \delta_{i}\right)$ are scaled points measured relative to $\kappa$ so they are non-zero distinct vectors (recall that $l_{P i C i}>0$ and $l_{C i P i+1}>0$ ), the sum of squares (C.1) becomes zero when

$$
\left(\eta_{i x}, \eta_{i y}\right) \cdot\left(z_{1}, z_{3}\right)=S_{i} z_{5} \quad \text { and } \quad\left(\eta_{i x}, \eta_{i y}\right) \cdot\left(z_{2}, z_{4}\right)=S_{i} z_{6}
$$

Note that we can define the speeds as $S_{i}=\left(\eta_{i x}, \eta_{i y}\right) \cdot\left(\frac{1}{2}\left(\xi_{i}+\xi_{i+1}\right), \frac{1}{2}\left(\psi_{i}+\psi_{i+1}\right)\right)$. The above equalities are then satisfied if either $\left(\eta_{i x}, \eta_{i y}\right)=\mathbf{0}$ (which is not the case) or

$$
\left(z_{1}, z_{3}\right)=\frac{1}{2}\left(\xi_{i}+\xi_{i+1}, \psi_{i}+\psi_{i+1}\right) z_{5} \quad \text { and } \quad\left(z_{2}, z_{4}\right)=\frac{1}{2}\left(\xi_{i}+\xi_{i+1}, \psi_{i}+\psi_{i+1}\right) z_{6} .
$$


Since the polygon has at least three distinct points, the only way that $\left(z_{1}, z_{3}\right)=\frac{1}{2}\left(\xi_{i}+\xi_{i+1}, \psi_{i}+\psi_{i+1}\right) z_{5}$ and $\left(z_{2}, z_{4}\right)=\frac{1}{2}\left(\xi_{i}+\xi_{i+1}, \psi_{i}+\psi_{i+1}\right) z_{6}$ for all $i \in\left\{1, \ldots, I_{\max }\right\}$, $I_{\max } \geq 3$, is if $\mathbf{z}=\mathbf{0}$. Therefore, $\mathbf{L}$ is positive definite, hence, invertible.

\section{Figure Captions}

Fig. 1 shows the states that come together at the vertex " $O$ ". Any number of states are permitted to come together at the vertex. The dashed lines show the zone boundaries. Note the labeling of the zone boundaries and the states. The unit vectors $\mathbf{n}_{i}$ separate states $\mathbf{U}_{i}$ and $\mathbf{U}_{i+1}$. The unit vectors $\mathbf{t}_{i}$ are orthogonal to $\mathbf{n}_{i}$ and have a counterclockwise orientation. The $1 D$ Riemann problem emanating from the boundary $\mathbf{n}_{1}$ is also shown.

Fig. 2 shows a minimalist wave model in the subsonic case, as originally proposed by Wendroff. The thick solid lines show shocks, the thick dotted lines show the contacts in the 1D Riemann problems. The vertices of the wave model are formed by the interaction of shocks from contiguous zone boundaries. The $i^{\text {th }}$ vertex is formed by the intersection of extremal shocks coming from the $i^{\text {th }}$ and $(i-1)^{\text {th }}$ zone boundaries.

Fig. 3 shows the speeds $S_{1}$ to $S_{5}$ associated with the five faces of the multidimensional wave model.

Fig. 4 shows the centroid of the multidimensional wave model. The big dot shows the centroid $k$. The coordinates $(x, y)$ are measured relative to the centroid $k$. The lengths of the sides of the multidimensional wave model are also shown and are used in our method to weight the shock jump conditions.

Fig. 5 shows a situation where the wave model construction described in Section II produces a non-convex wave model (thick black lines). It is important to ensure that the polygon that bounds the wave model is convex. This is achieved by picking a slightly larger wave model (thick grey lines).

Fig. 6a shows the density from the Sod shock test problem along with the mesh structure, while fig. $6 b$ plots out the density in one dimension along with the reference solution. Fig. $6 c$ shows the density from the Lax shock test problem along with the mesh structure, while fig. $6 \mathrm{~d}$ plots out the density in one dimension along with the reference solution.

Fig. 7a shows the density profile at the final time when the multidimensional Riemann solver with self-similar sub-structure from this paper is used. Fig. $7 b$ shows the corresponding density profile as a one-dimensional plot.

Figs. $8 a$ and $8 b$ show the densities from the first and second multidimensional Riemann problems. Only the lower left portion of the computational domain is shown in Fig. $8 b$. 
In Fig. 9 we show the density variable from a simulation of the double Mach reflection problem Fig. 9a shows entire density variable while Fig. $9 b$ shows a zoom-in of the vortex sheet.

Figs. 10a, 10b, 10c and 10d show the density, gas pressure, Mach number and magnetic pressure for the MHD Rotor problem. The MuSIC Riemann solver with least square projection that was based on the one-dimensional HLLC Riemann solver was used.

Figs. 11a, 11b, 11c and 11d show the density, gas pressure, Mach number and magnetic pressure for the MHD Orszag-Tang problem. The MuSIC Riemann solver with least square projection that was based on the one-dimensional HLLC Riemann solver was used.

Fig. 12 shows the evolution of normalized magnetic energy for the field loop problem. Fig. 12a shows the magnetic energy as a function of time, where the magnetic energy is normalized by the initial magnetic energy. Fig. $12 b$ shows the magnetic energy in the axial component of the magnetic field, which is also normalized by the initial magnetic energy. A third order ADERWENO scheme with multidimensional Riemann solver was used.

Figs. 13a through 13d show the density, pressure, magnitude of the velocity and magnitude of the magnetic field respectively in the midplane of the three-dimensional MHD blast wave simulation. 


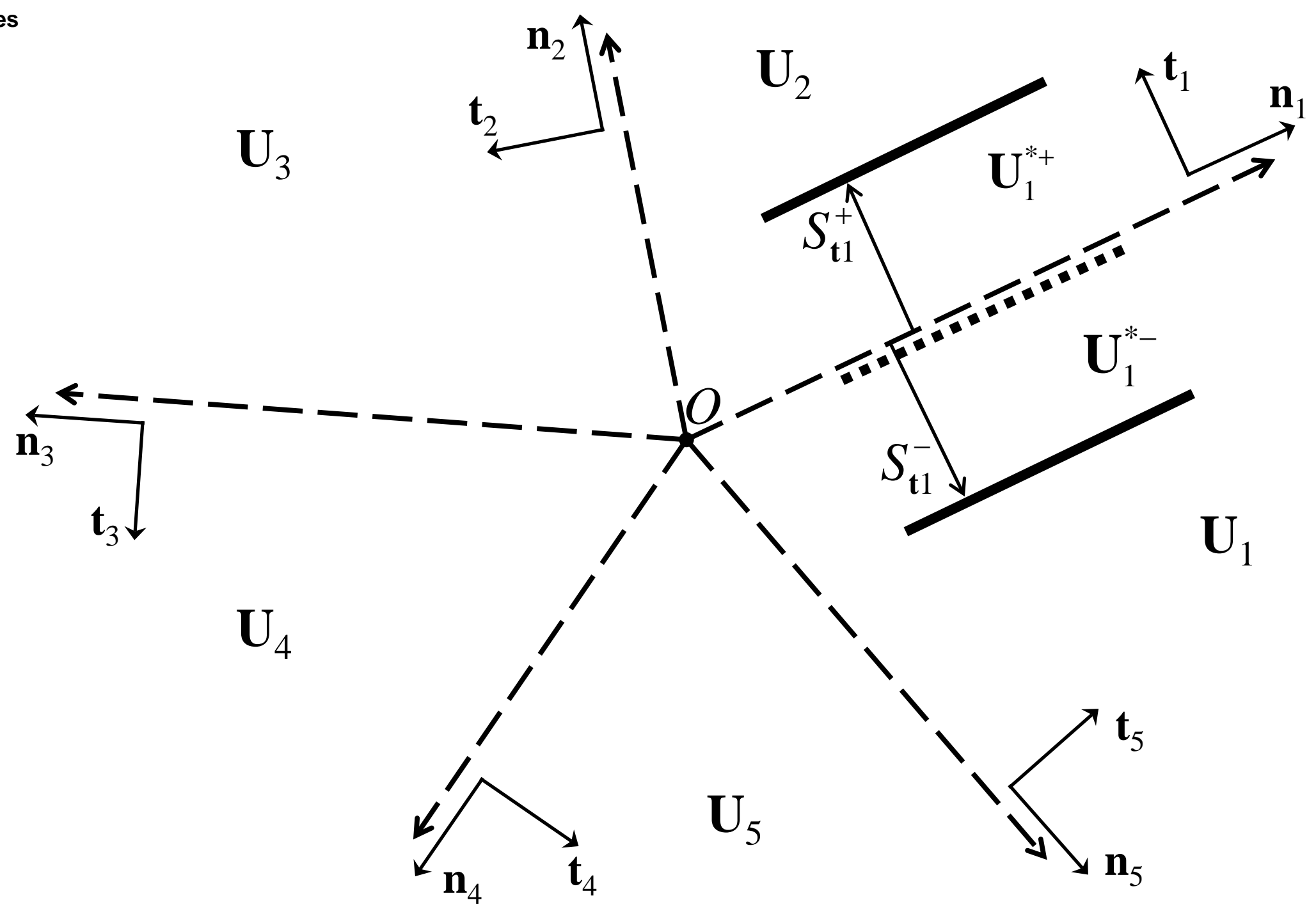

Fig. 1 shows the states that come together at the vertex "O". Any number of states are permitted to come together at the vertex. The dashed lines show the zone boundaries. Note the labeling of the zone boundaries and the states. The unit vectors $\mathbf{n}_{i}$ separate states $\mathbf{U}_{i}$ and $\mathbf{U}_{i+1}$. The unit vectors $\mathbf{t}_{i}$ are orthogonal to $\mathbf{n}_{i}$ and have a counterclockwise orientation. The $1 D$ Riemann problem emanating from the boundary $\mathbf{n}_{1}$ is also shown. 


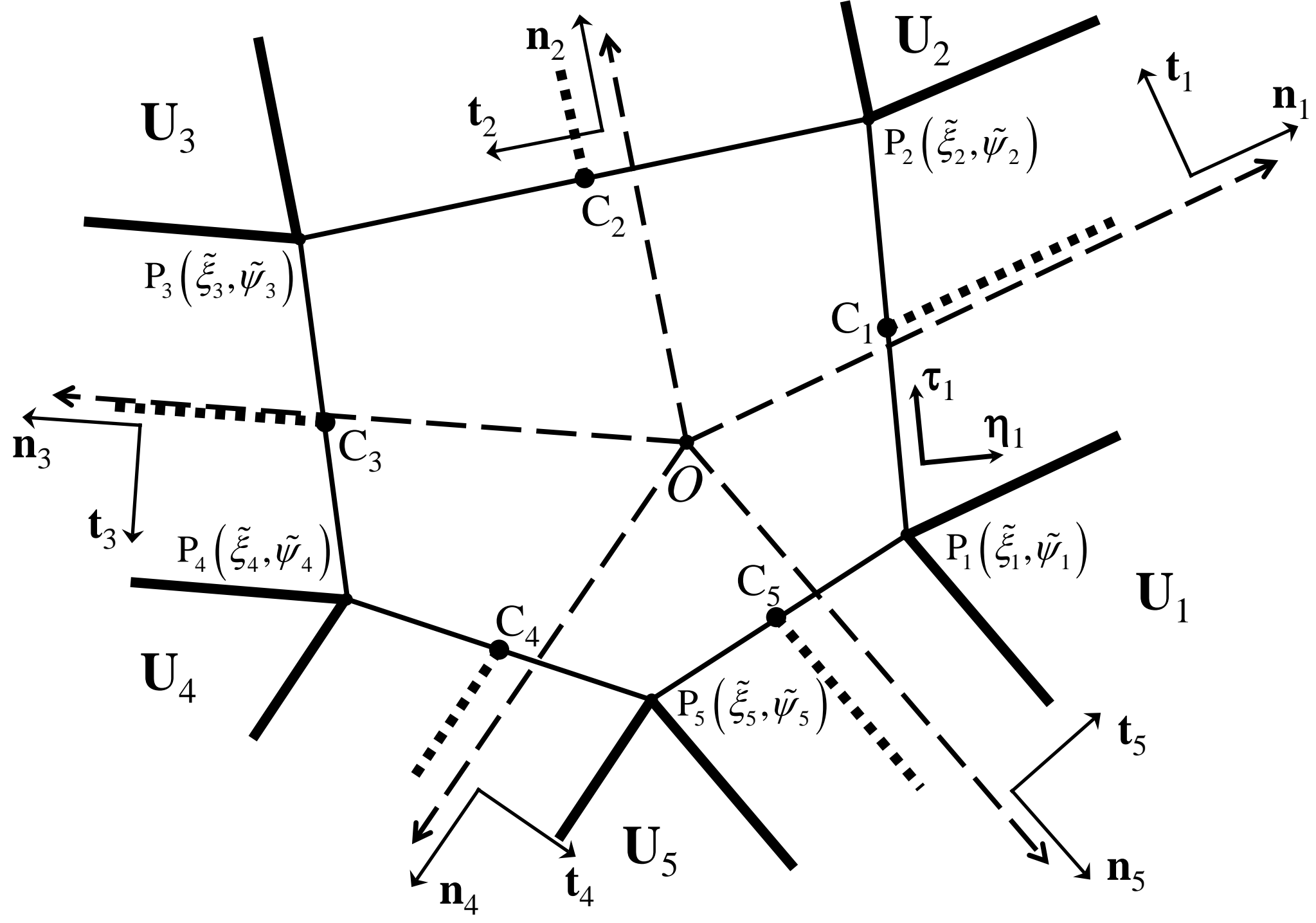

Fig. 2 shows a minimalist wave model in the subsonic case, as originally proposed by Wendroff. The thick solid lines show shocks, the thick dotted lines show the contacts in the 1D Riemann problems. The vertices of the wave model are formed by the interaction of shocks from contiguous zone boundaries. The $i^{\text {th }}$ vertex is formed by the intersection of extremal shocks coming from the $i^{\text {th }}$ and $(i-1)^{\text {th }}$ zone boundaries. 


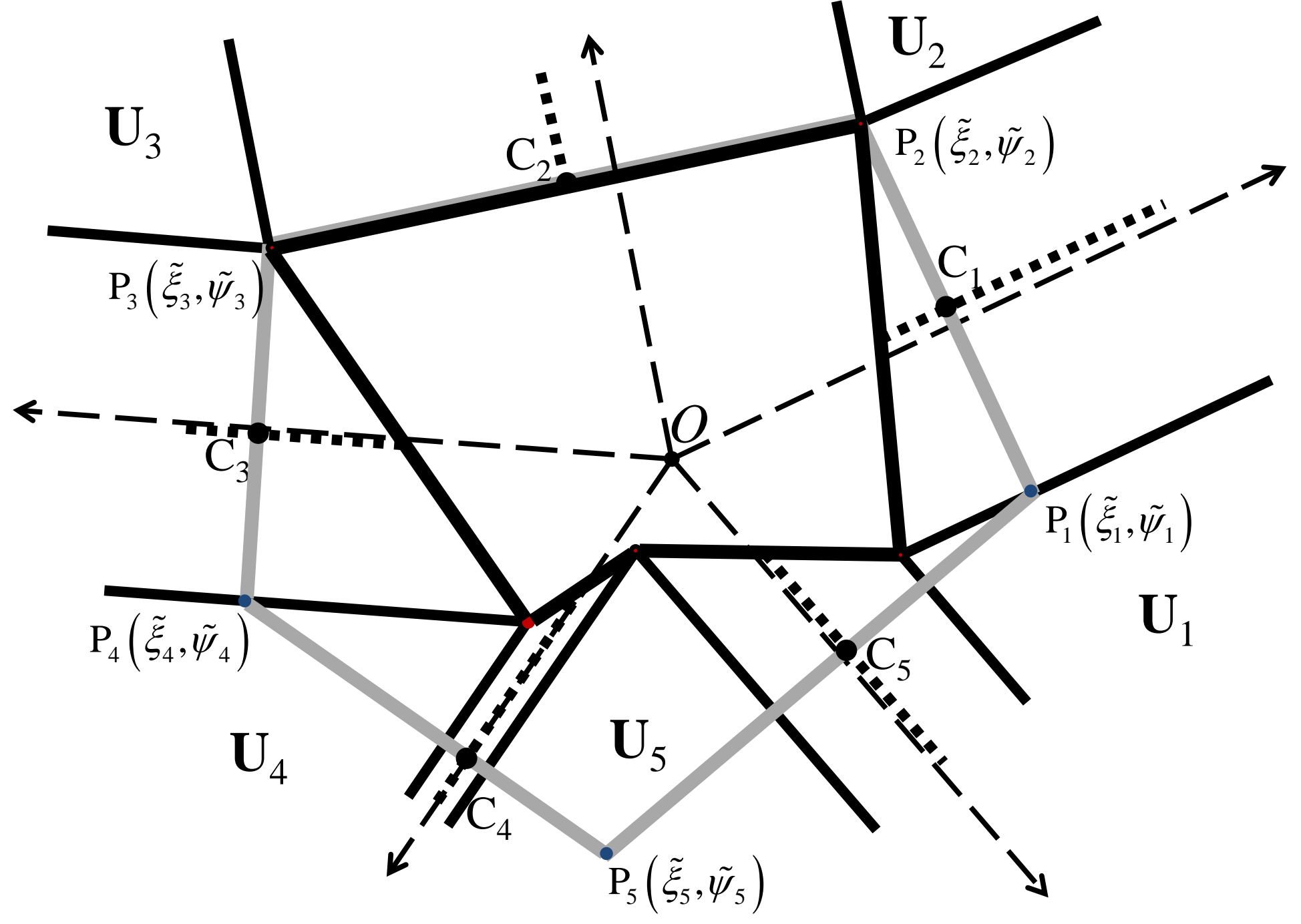

Fig. 5 shows a situation where the wave model construction described in Section II produces a non-convex wave model (thick black lines). It is important to ensure that the polygon that bounds the wave model is convex. This is achieved by picking a slightly larger wave model (thick grey lines). 
a)

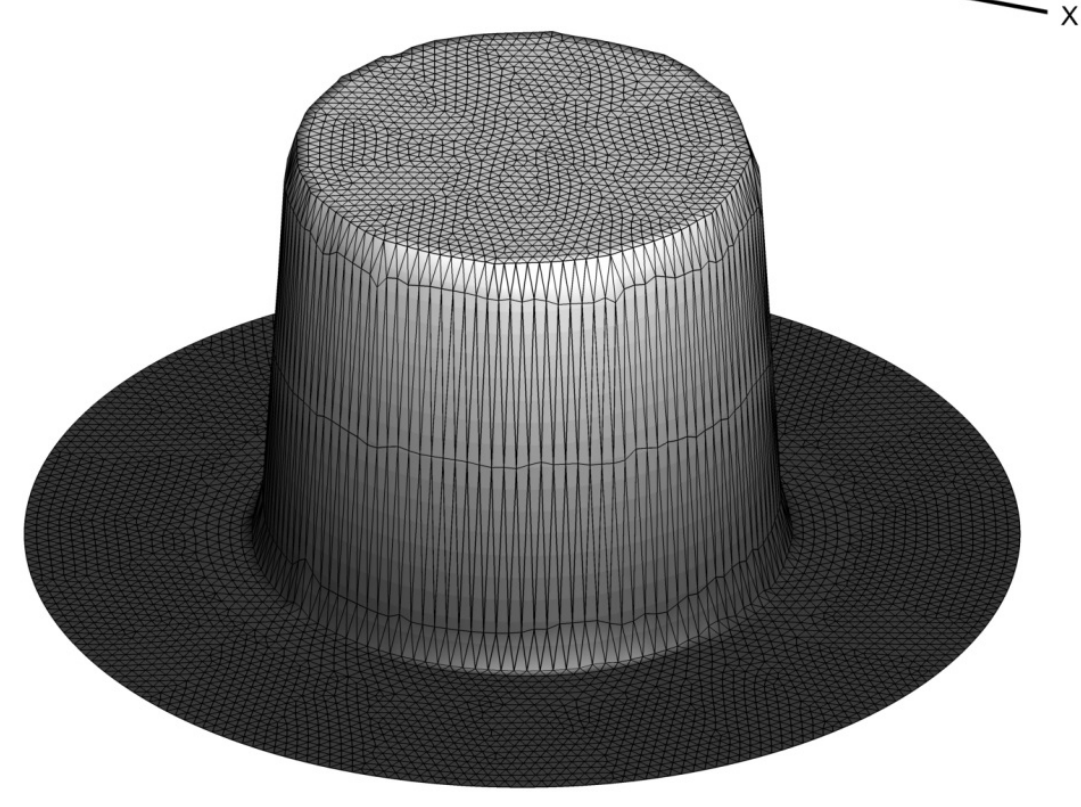

b)

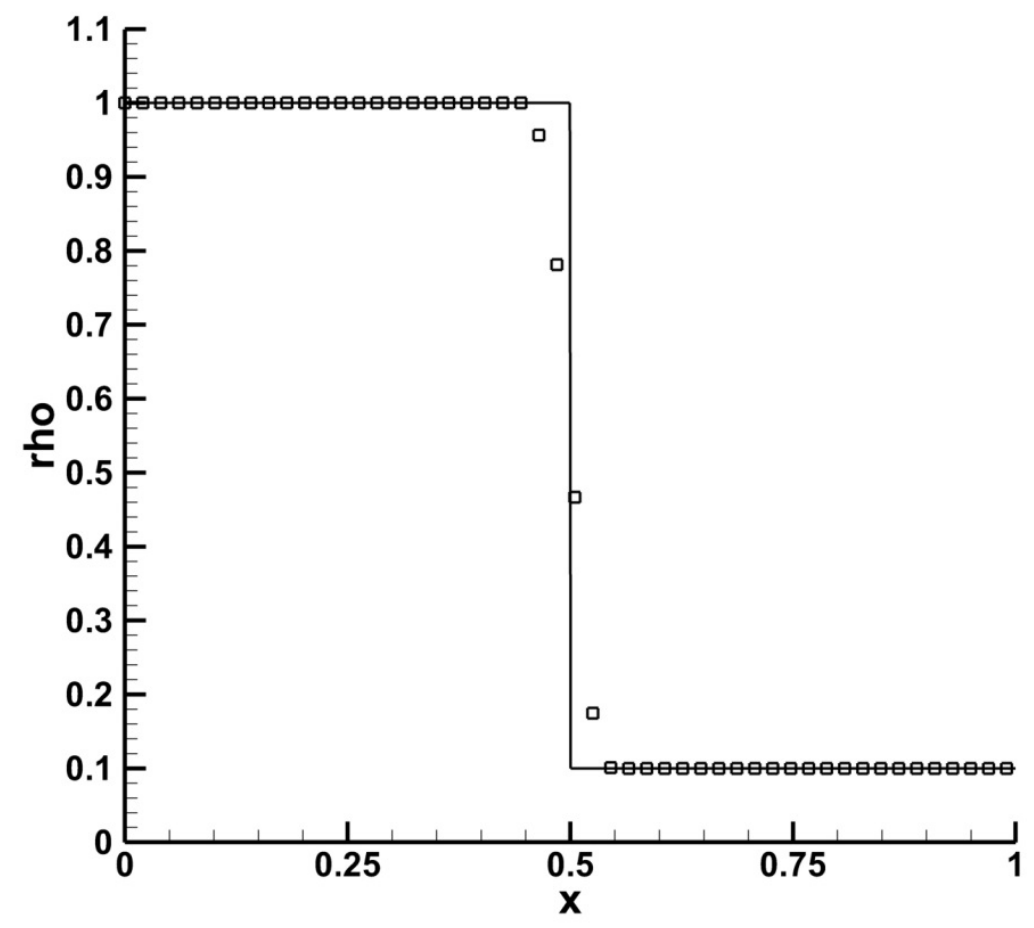

Fig. 7a shows the density profile at the final time when the multidimensional Riemann solver with self-similar sub-structure from this paper is used. Fig. $7 b$ shows the corresponding density profile as a one-dimensional plot. 
a)

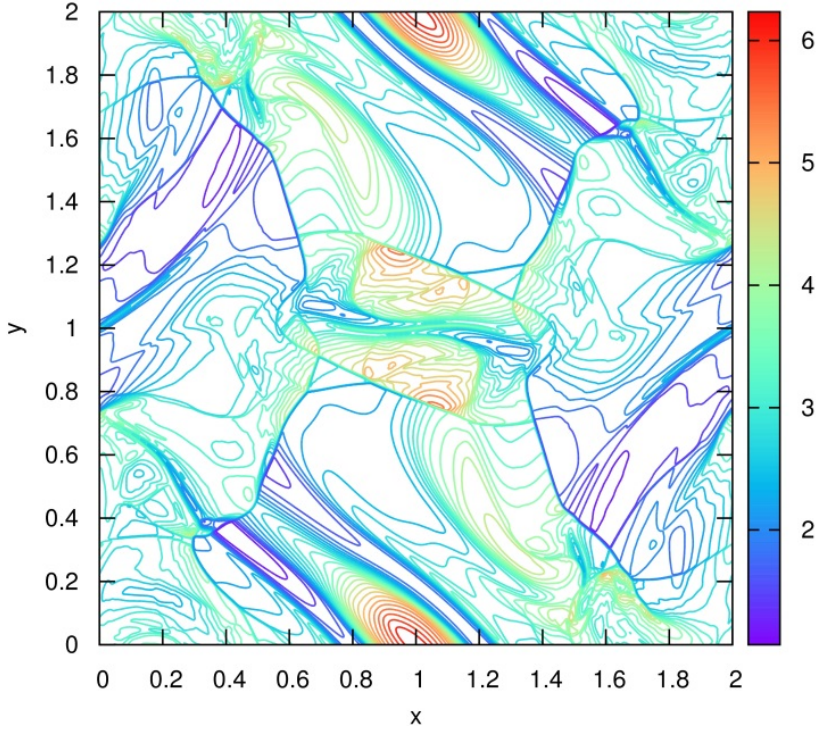

c)

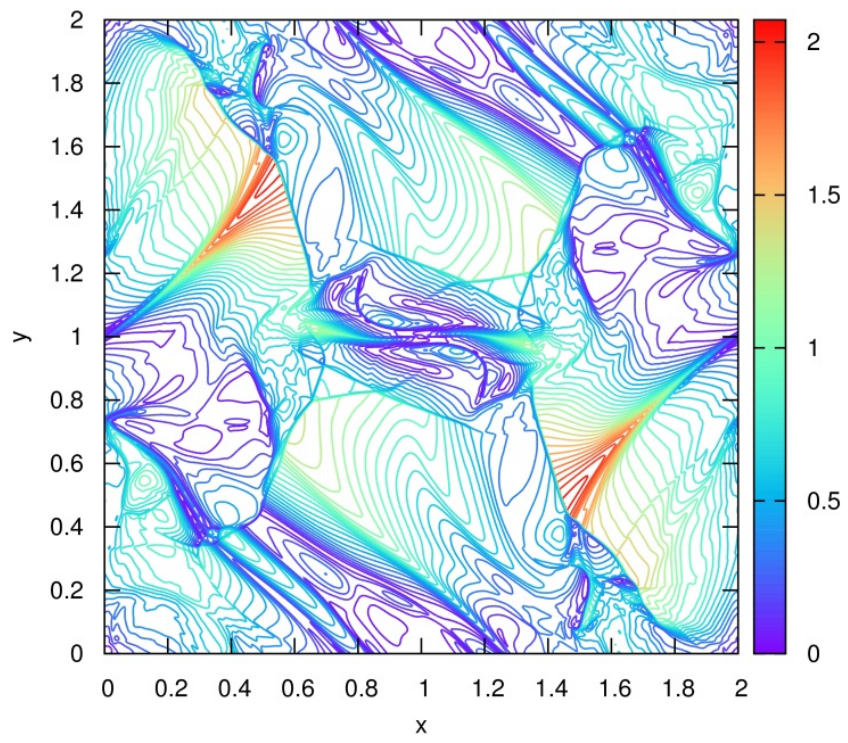

b)

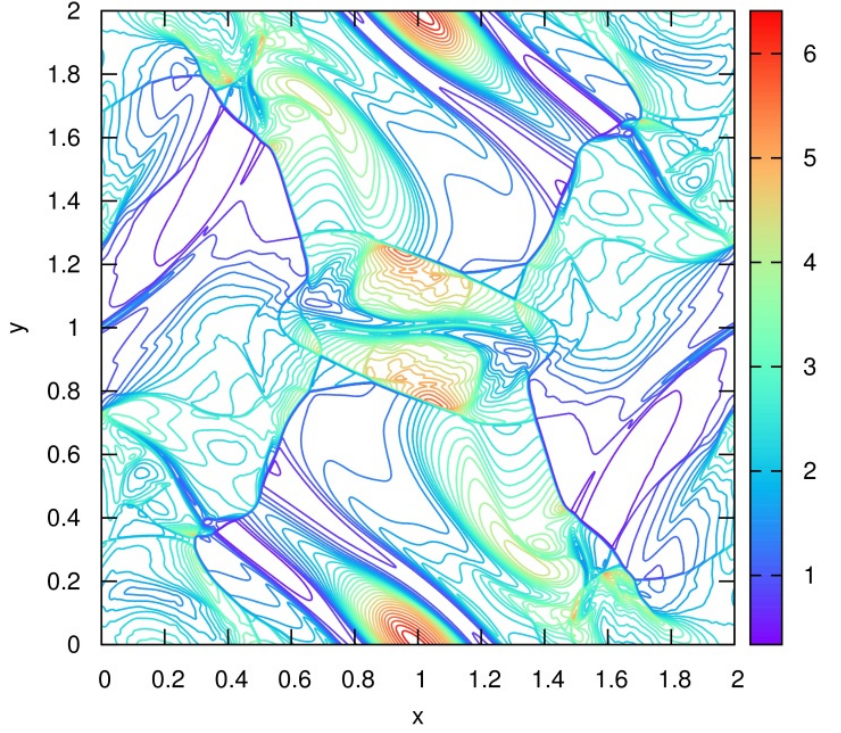

d)

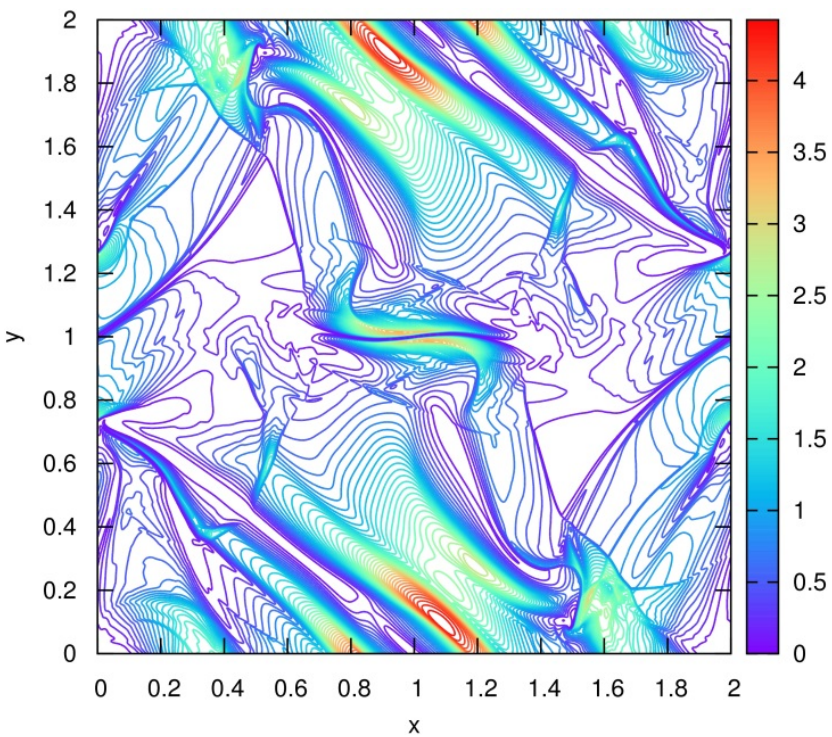

Figs. 11a, 11b, 11c and 11d show the density, gas pressure, Mach number and magnetic pressure for the MHD Orszag-Tang problem. The MuSIC Riemann solver with least square projection that was based on the one-dimensional HLLC Riemann solver was used. 
a)

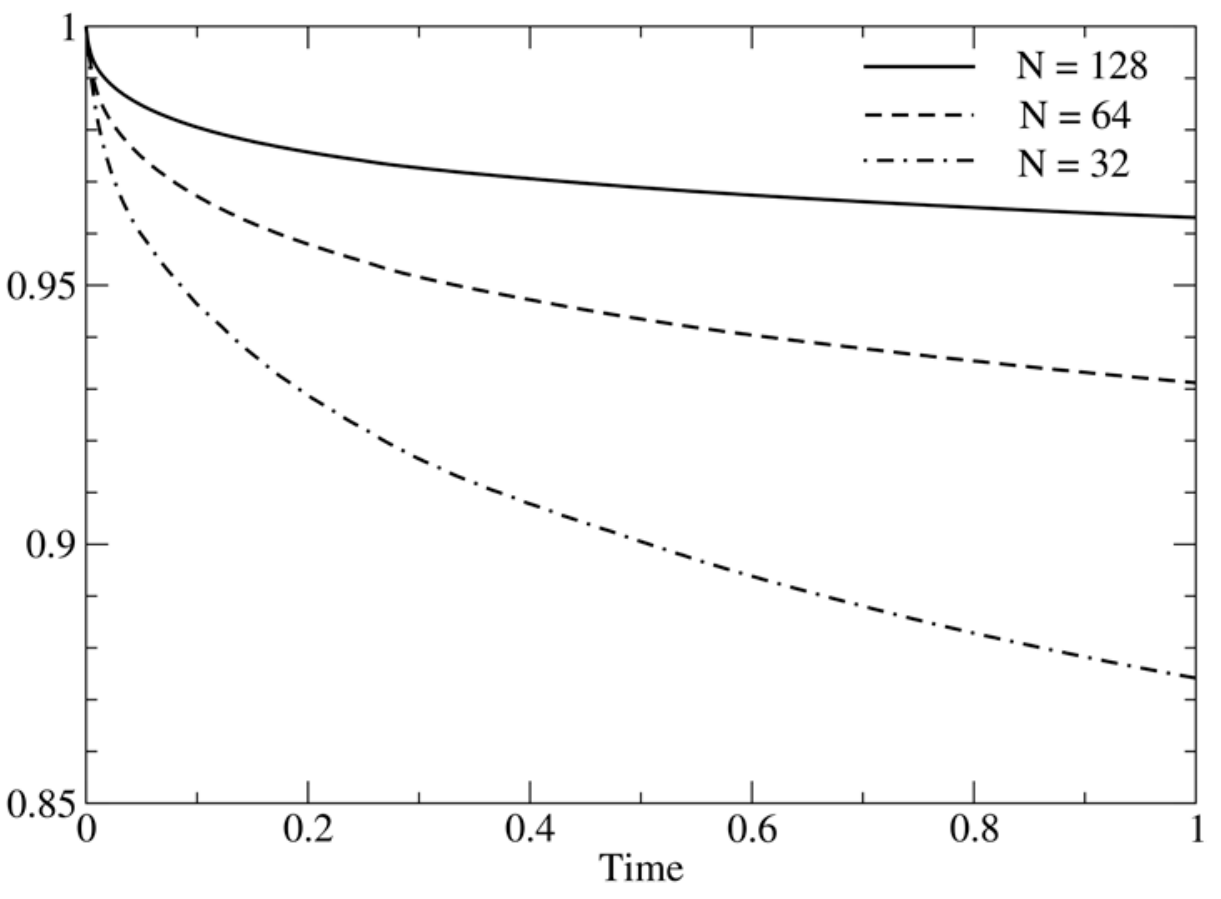

b)

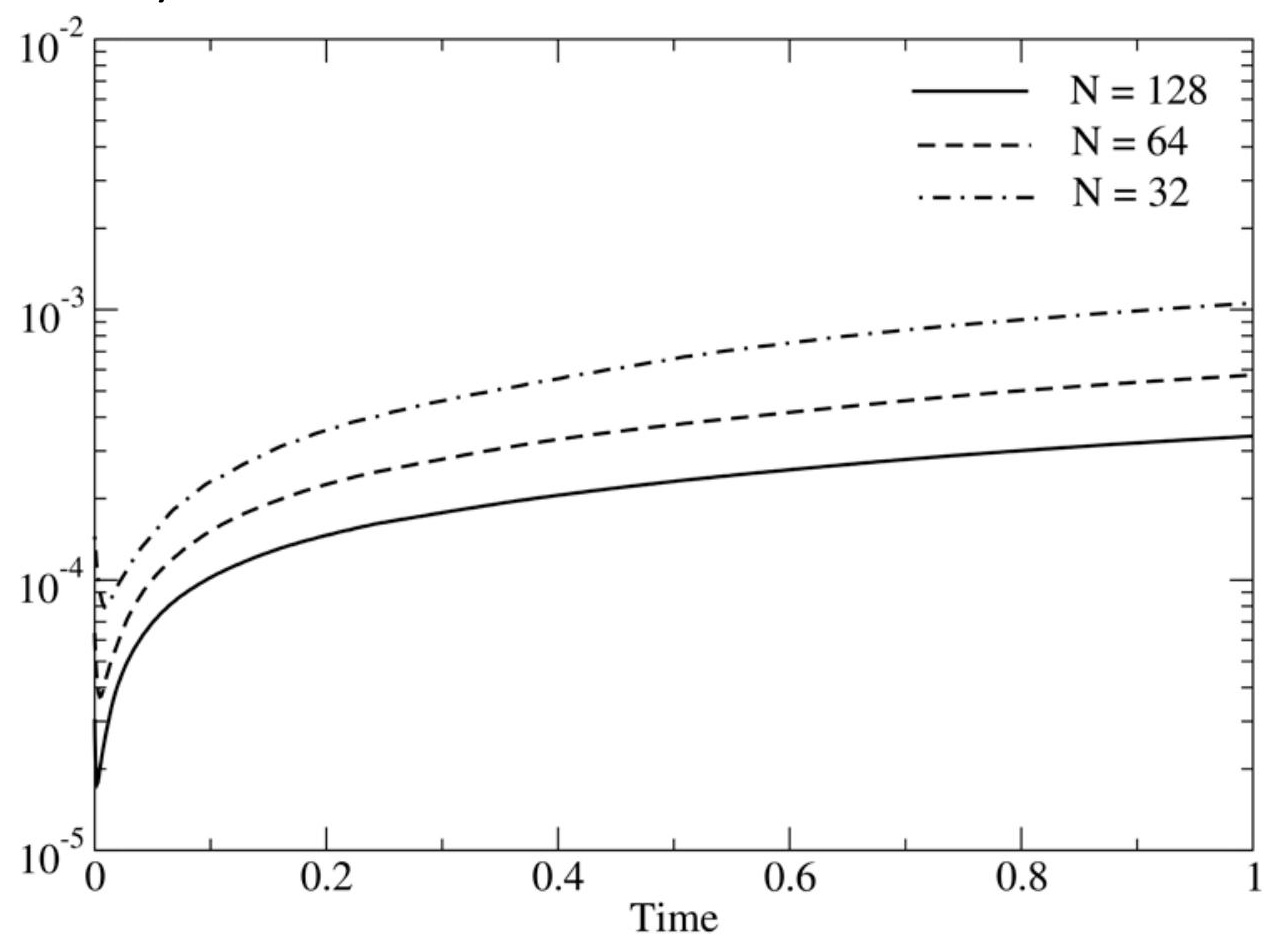

Fig. 12 shows the evolution of normalized magnetic energy for the field loop problem. Fig. 12a shows the magnetic energy as a function of time, where the magnetic energy is normalized by the initial magnetic energy. Fig. $12 b$ shows the magnetic energy in the axial component of the magnetic field, which is also normalized by the initial magnetic energy. A third order ADER-WENO scheme with multidimensional Riemann solver was used. 
a)

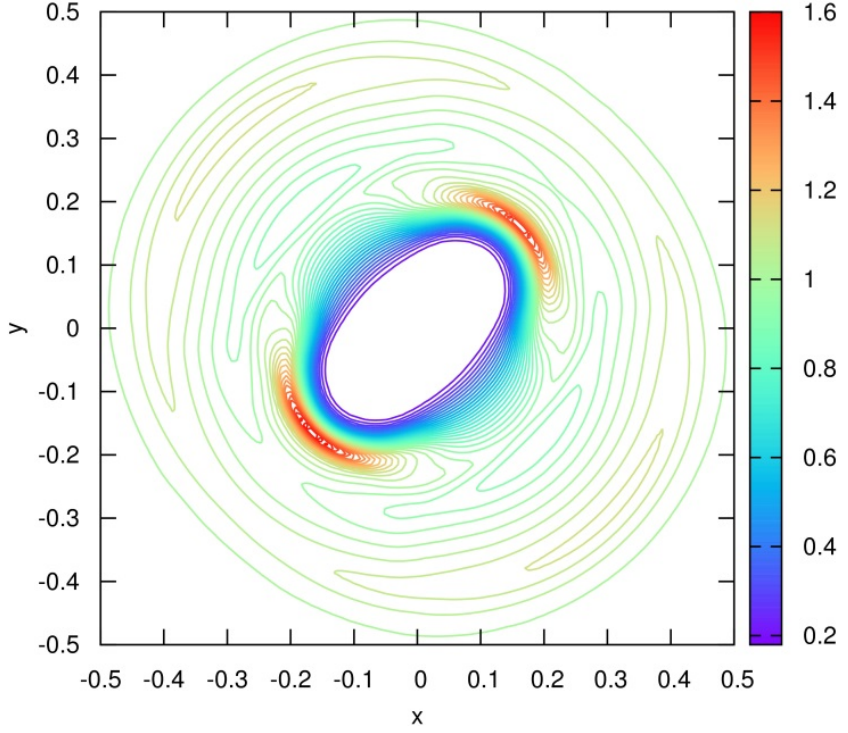

b)

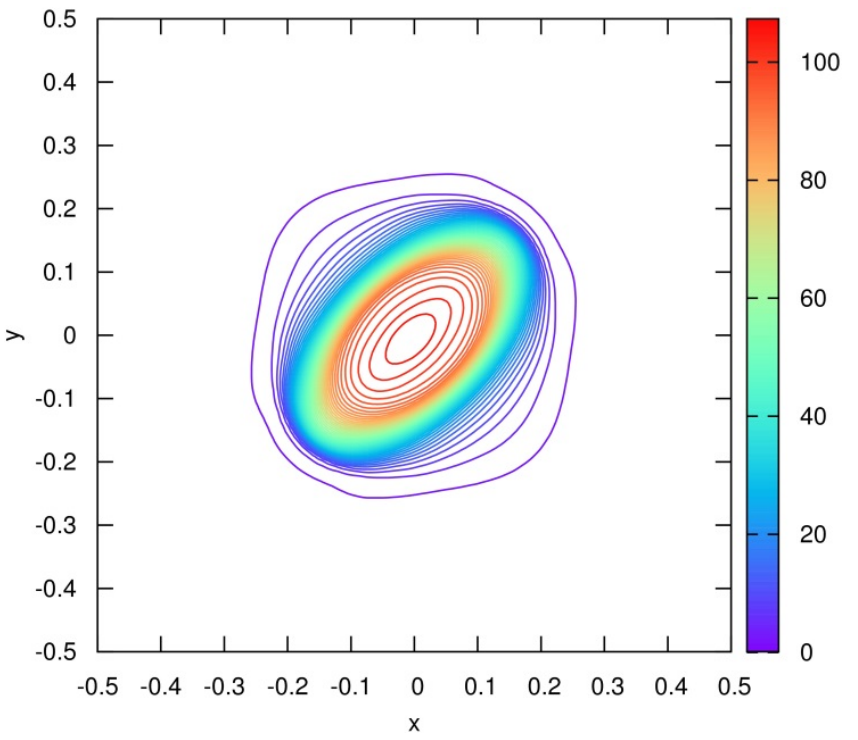

c)

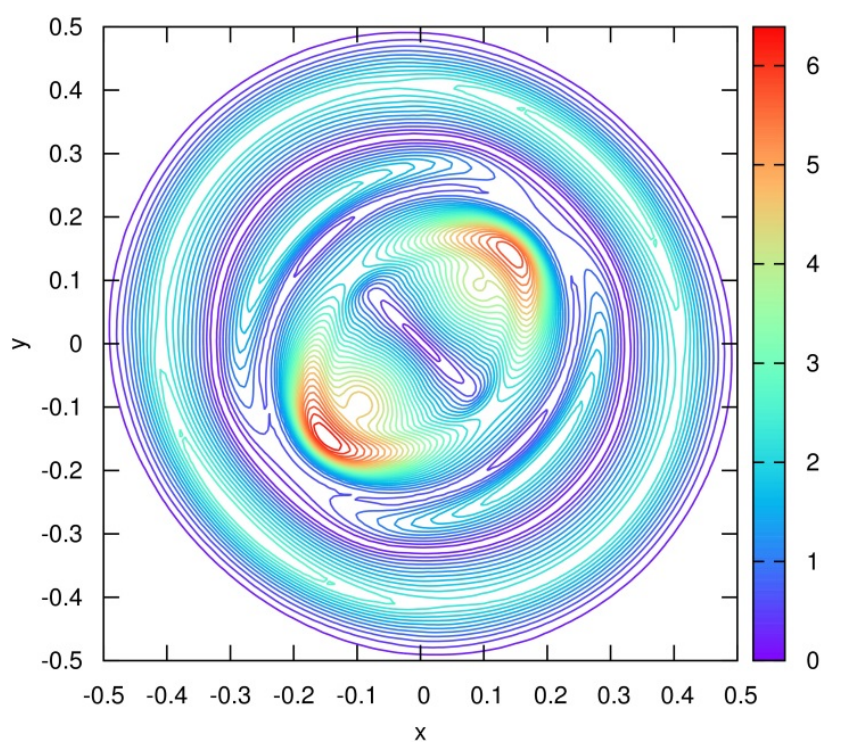

d)

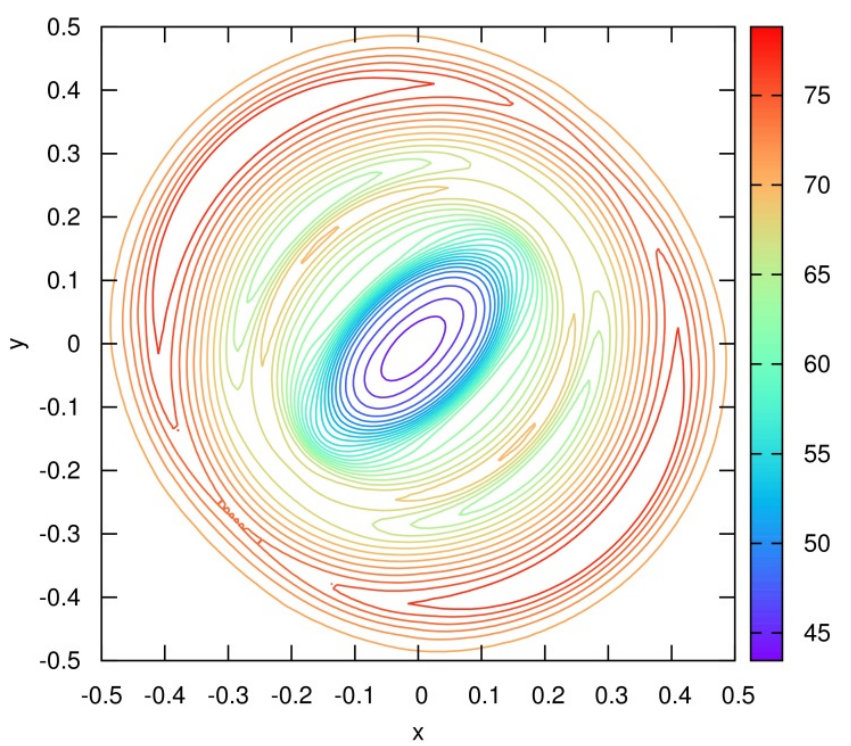

Figs. 13a through $13 d$ show the density, pressure, magnitude of the velocity and magnitude of the magnetic field respectively in the midplane of the three-dimensional MHD blast wave simulation. 


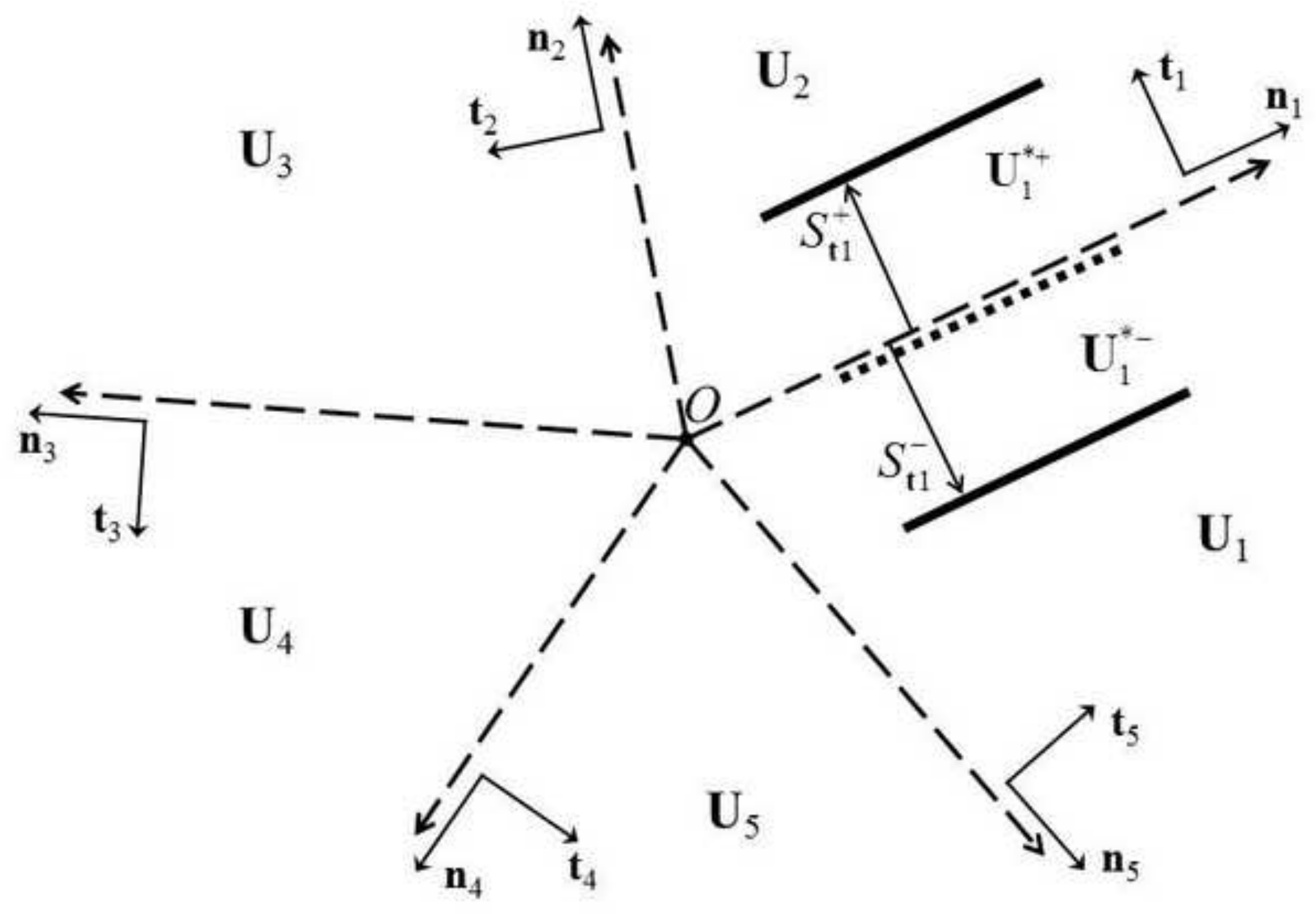

Fig. 1 shows the states that come together at the vertex " $O$ ". Any number of states are permitted to come together at the vertex. The dashedlines show the zone boundaries. Note the labeling of the zone boundaries and the states. The unitvectors $\mathbf{n}_{i}$ separate states $\mathbf{U}_{i}$ and $\mathbf{U}_{i+1}$. The unitvectors $\mathbf{t}_{i}$ are orthogonal to $\mathbf{n}_{i}$ and have a counterclockwise orientation. The $1 D$ Riemann problem emanating from the boundary $\mathbf{n}_{1}$ is also shown. 


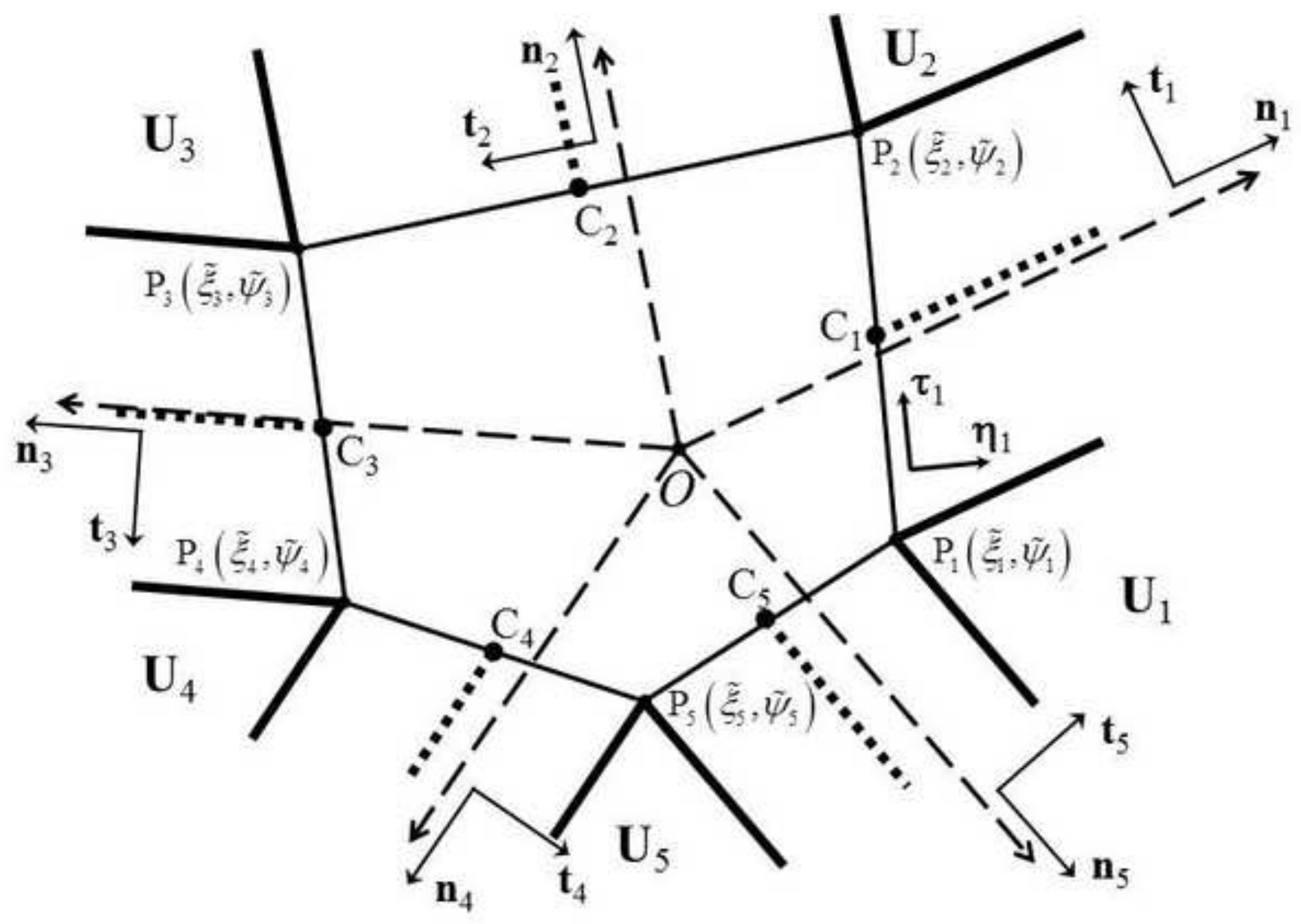

Fig. 2 shows a minimalist wave model in the subsonic case, as originally proposed by Wendroff. The thick solid lines show shocks, the thick dottedlines show the contacts in the $1 D$ Riemann problems. The vertices of the wave model are formed by the interaction of shocks from contiguous zone boundaries. The $i^{\text {th }}$ vertex is formed by the intersection of extremal shocks coming from the $i^{\text {th }}$ and $(i-1)^{\text {th }}$ zone boundaries. 


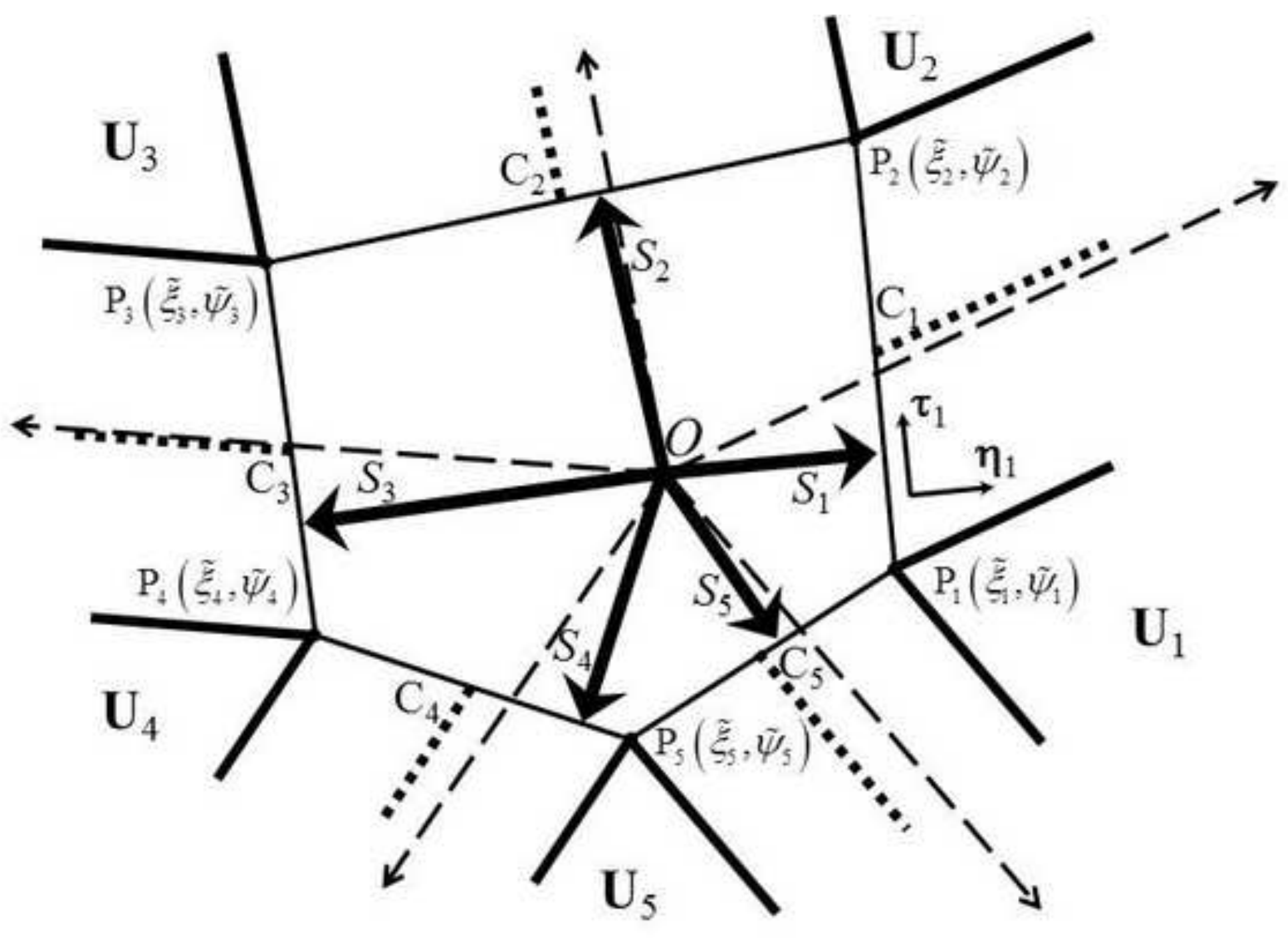

Fig. 3 shows the speeds $S_{1}$ to $S_{5}$ associated with the five faces of the multidimensional wave model. 


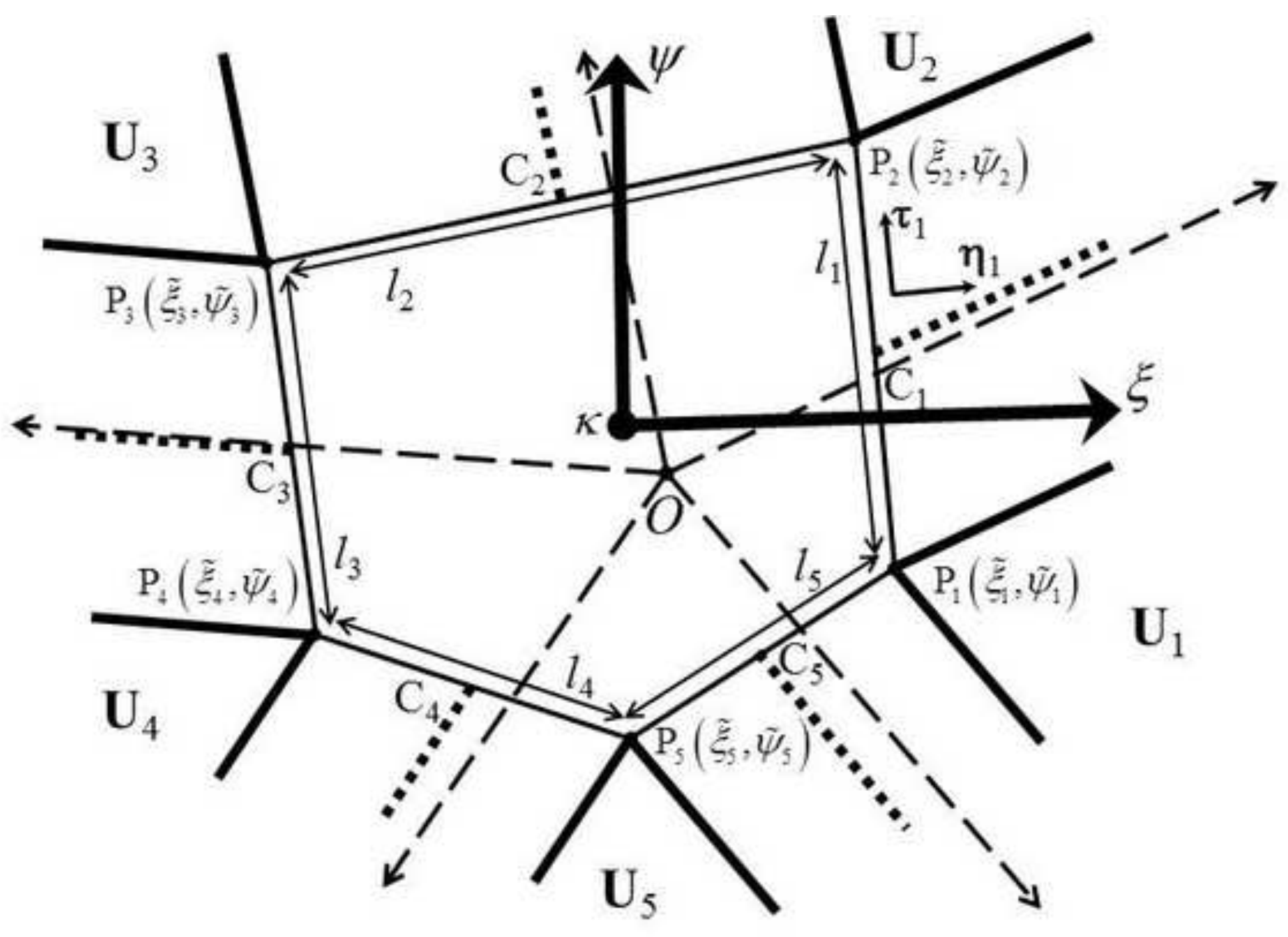

Fig. 4 shows the centroid of the multidimensional wave model. The big dot shows the centroid $\kappa$. The coordinates $(\xi, \psi)$ are measured relative to the centroid $\kappa$. The lengths of the sides of the multidimensional wave model are also shown and are used in our method to weight the shock jump conditions. 


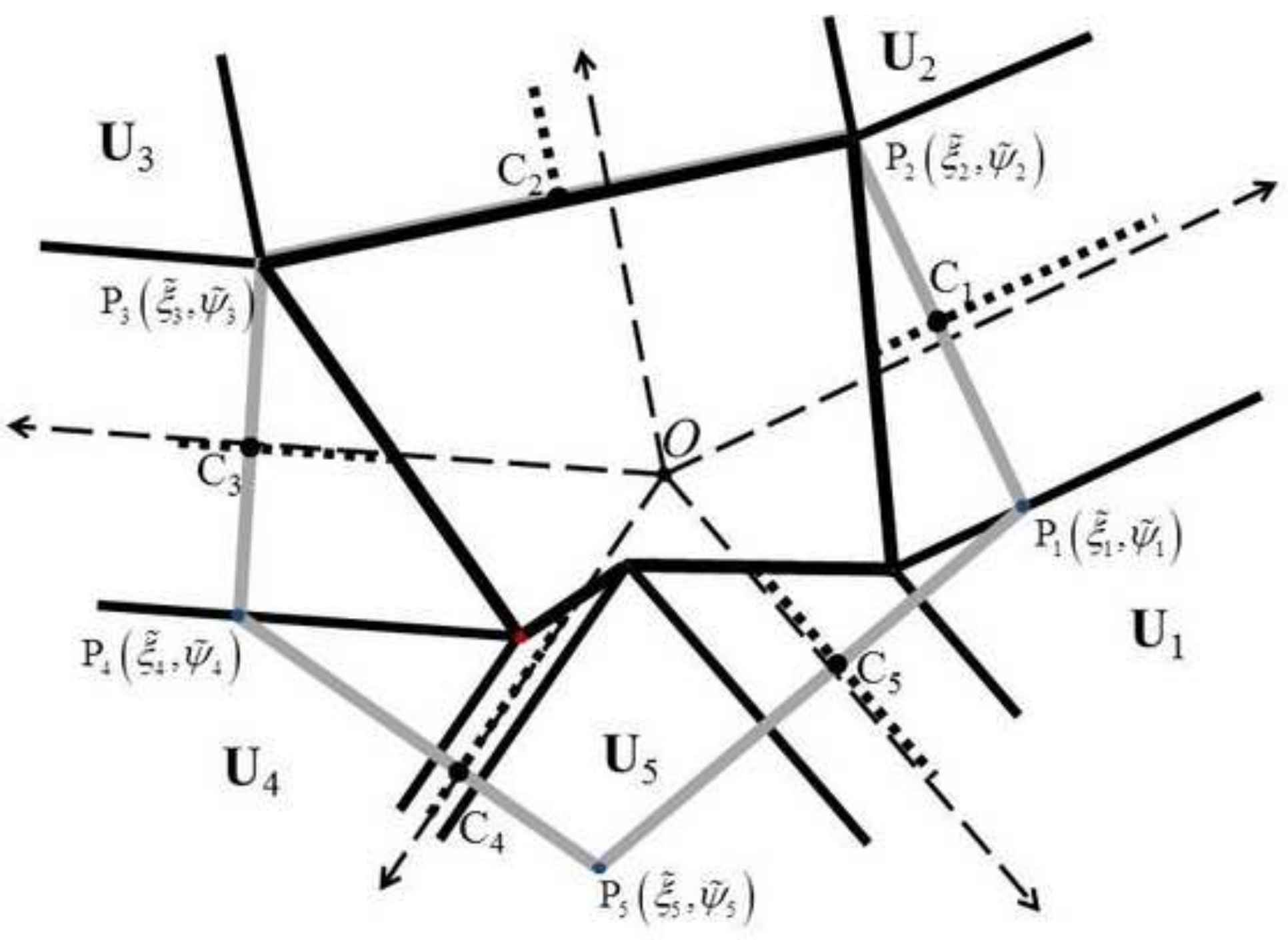

Fig. 5 shows a situation where the wave model construction described in Section II produces a non-convex wave model (thick black lines). It is important to ensure that the polygon that bounds the wave model is convex. This is achieved by picking a slightly larger wave model (thick greylines). 


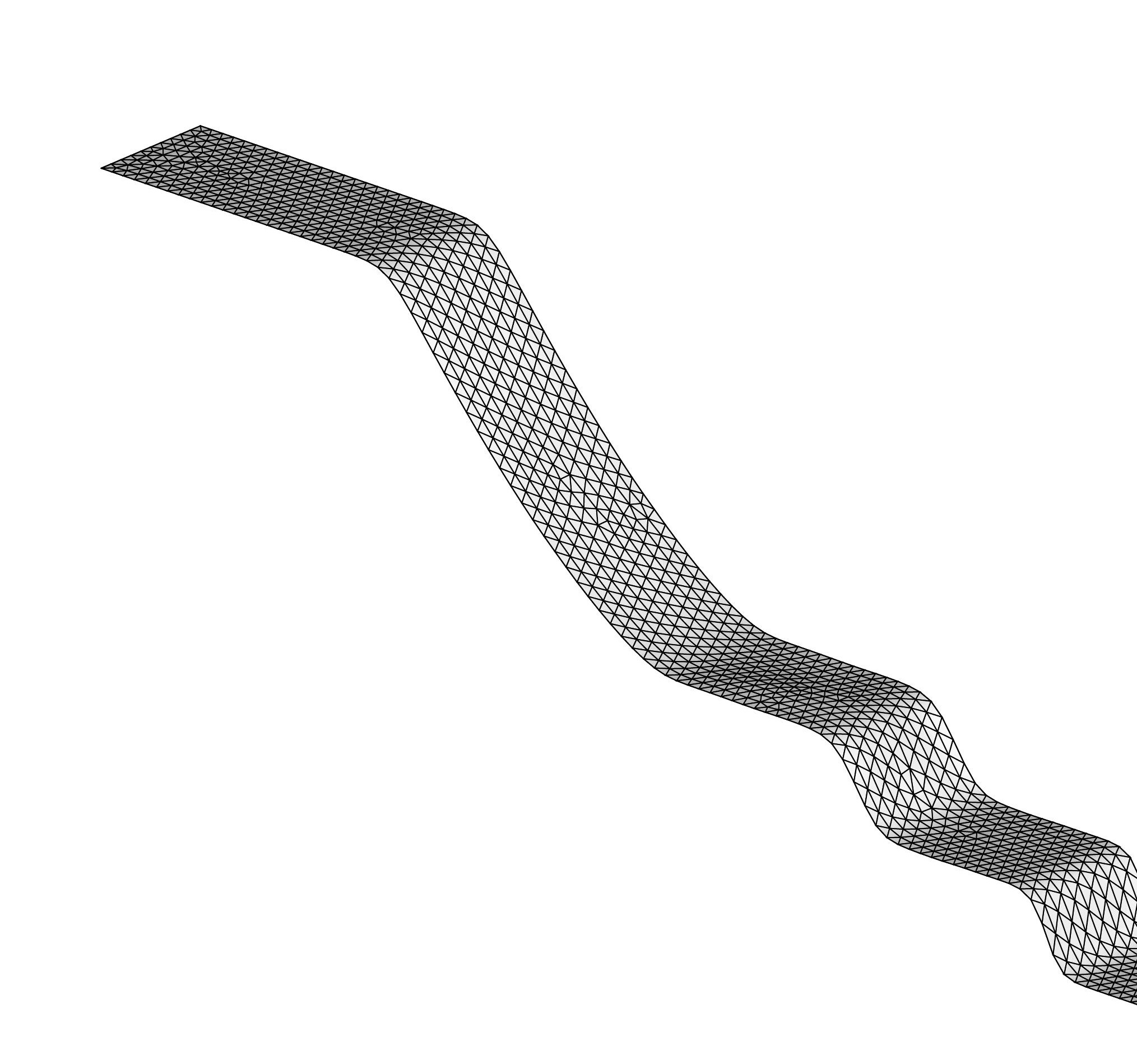

\section{.

.
.
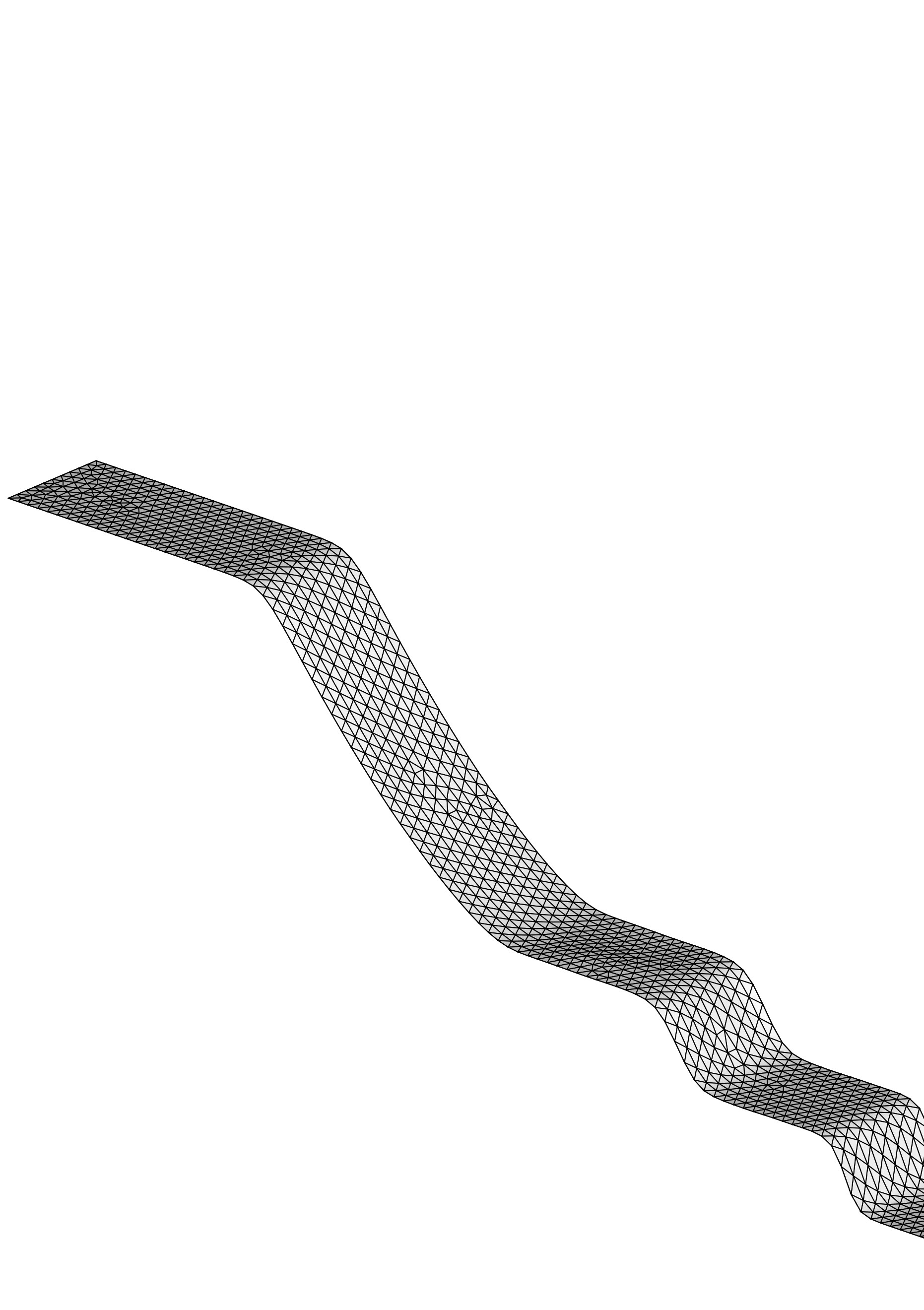


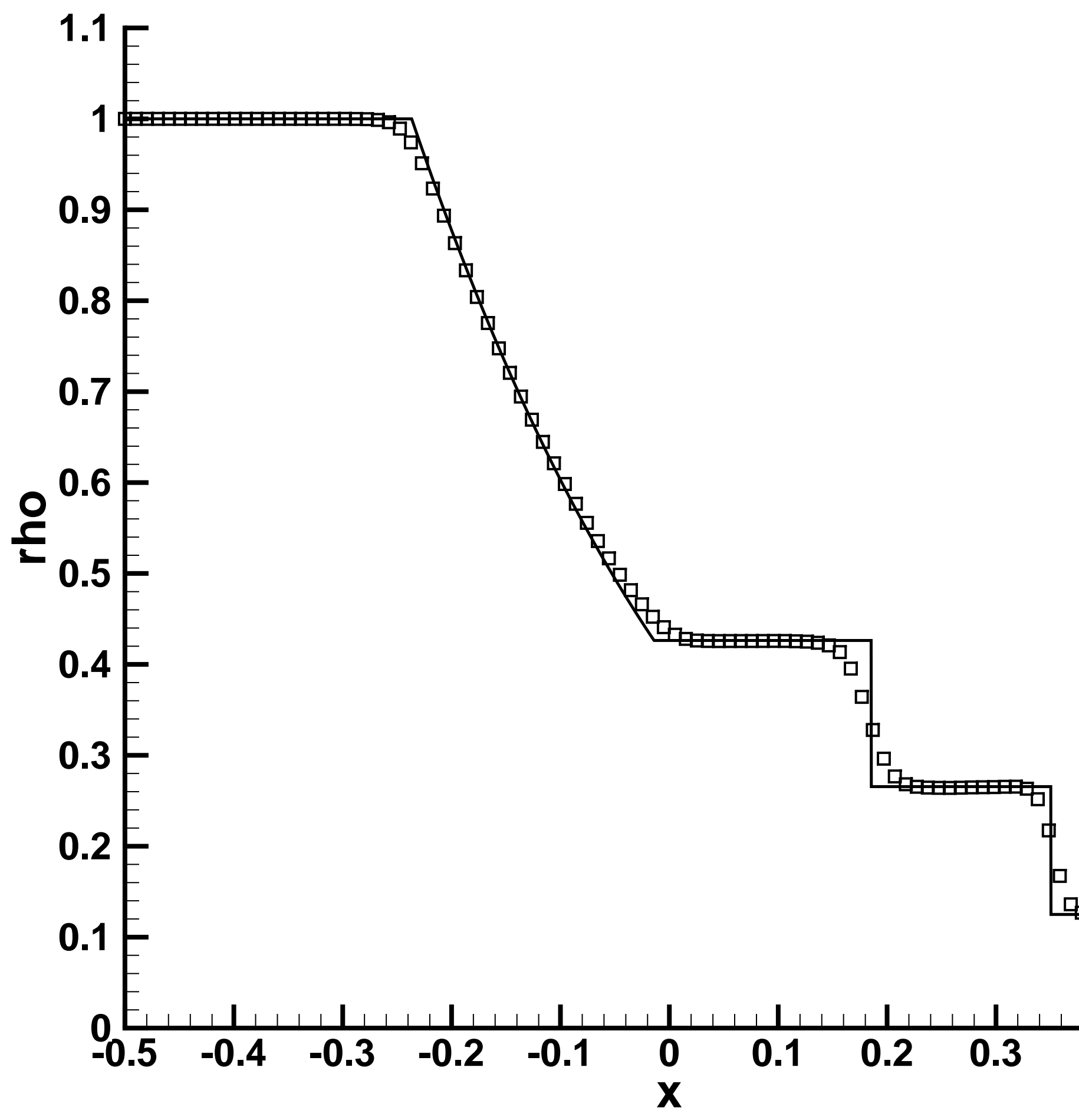




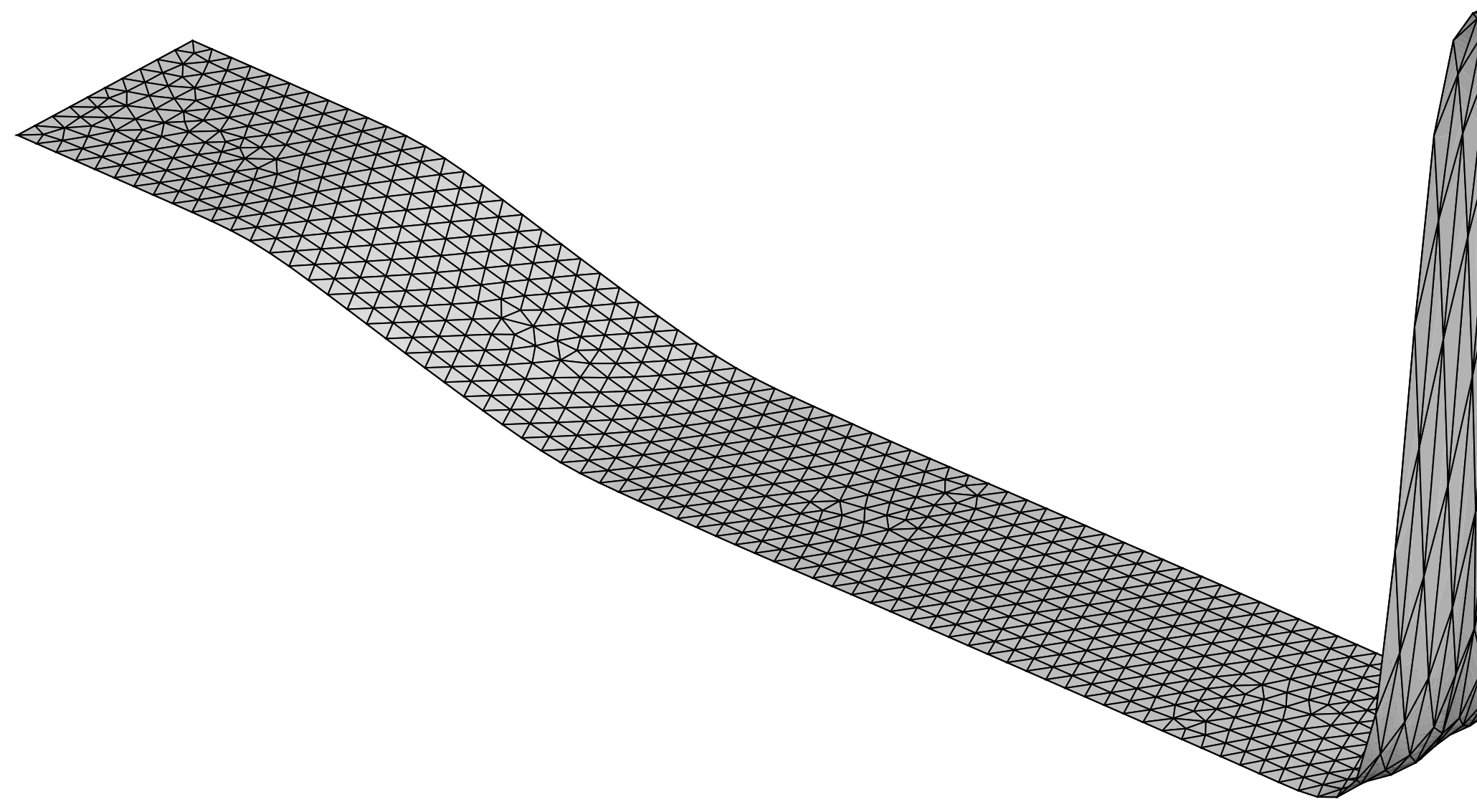



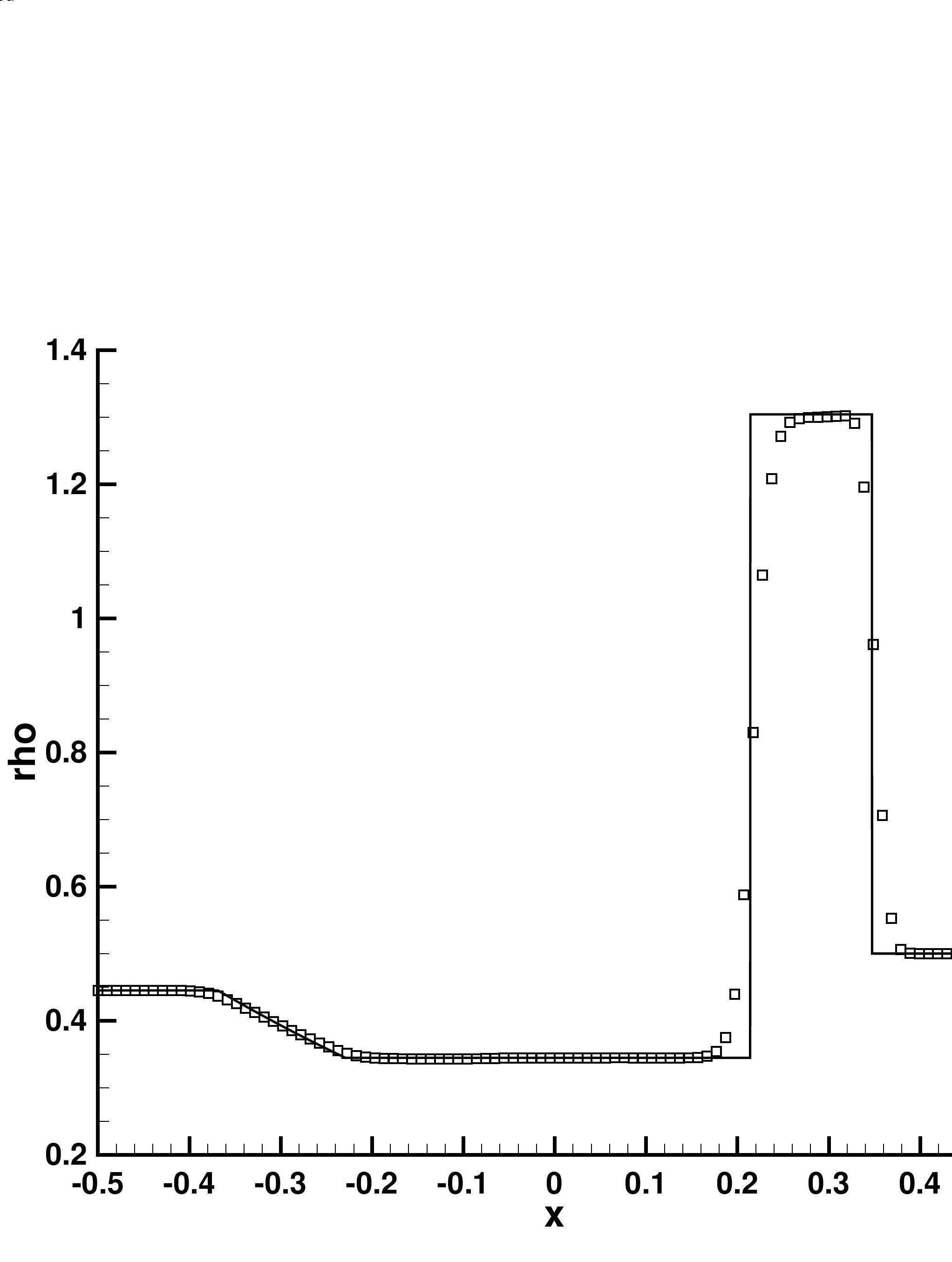


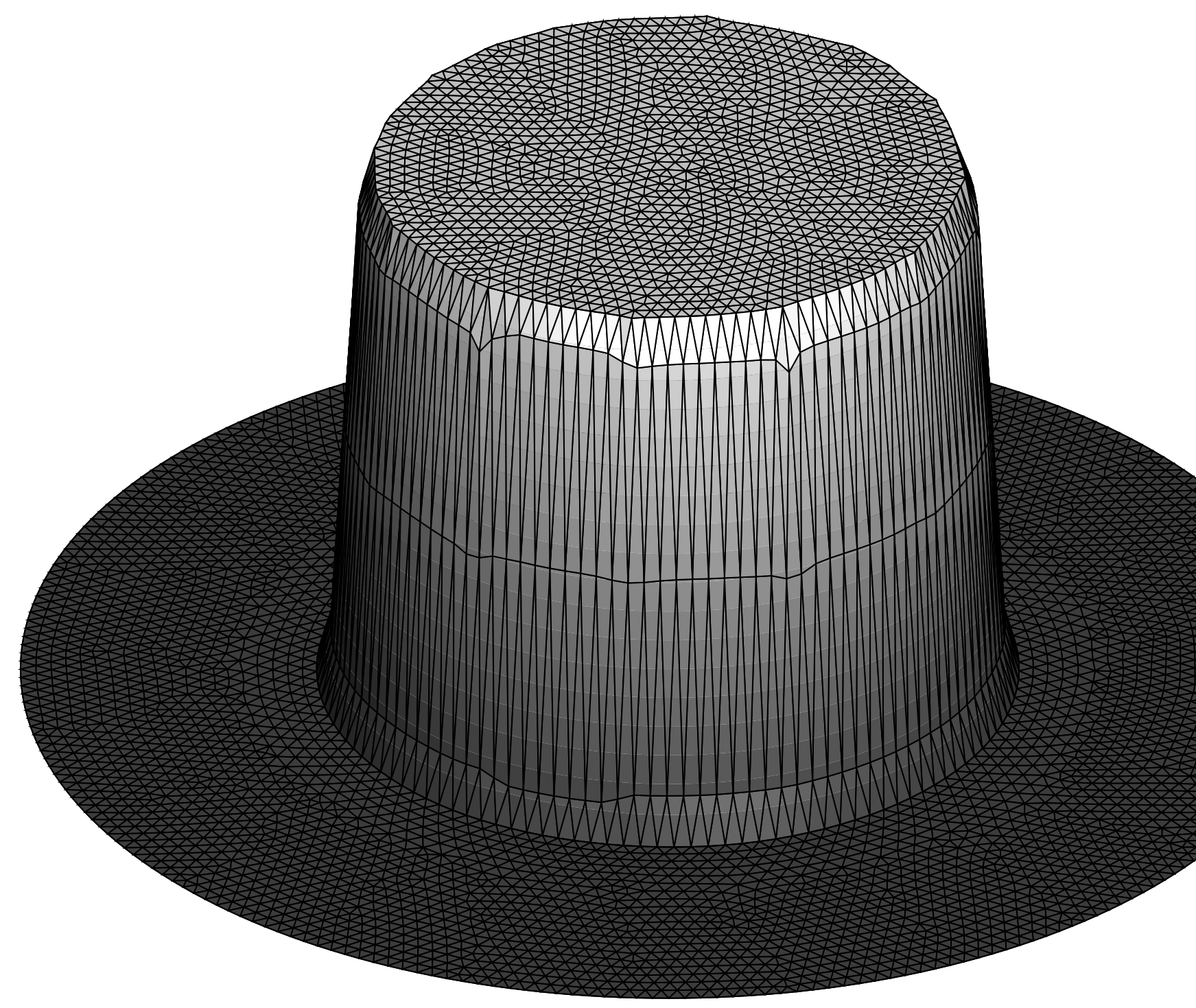



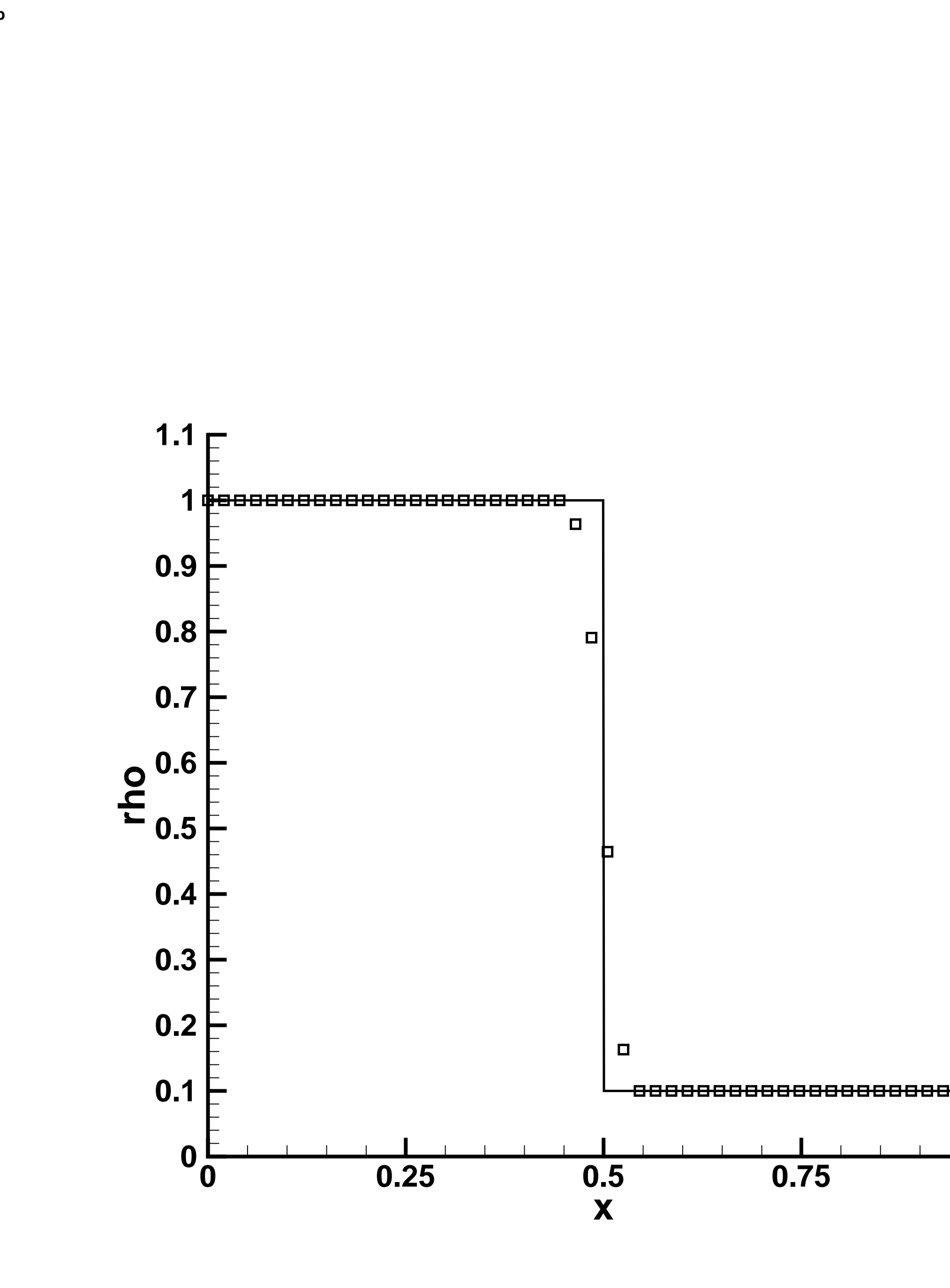

-




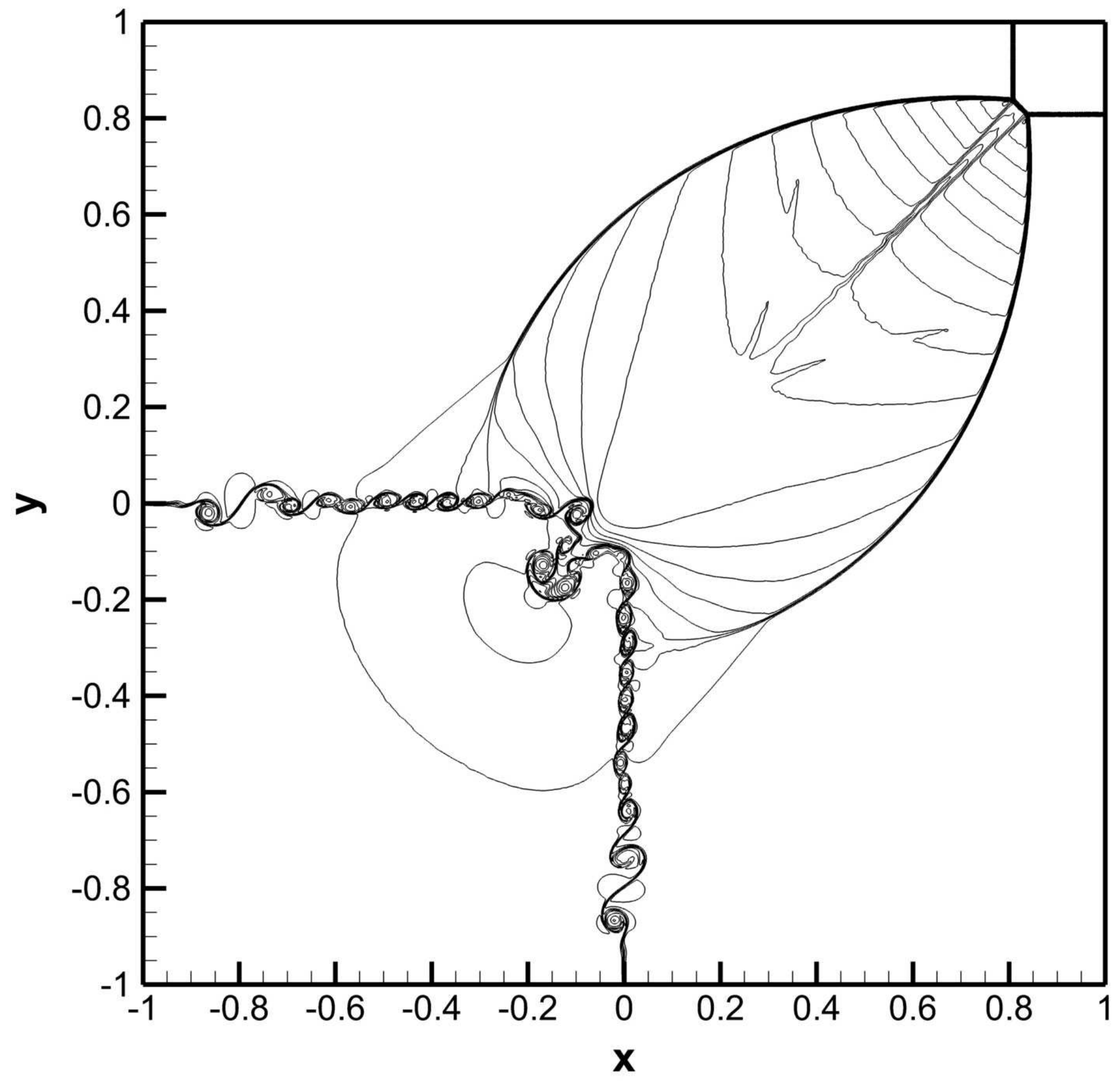




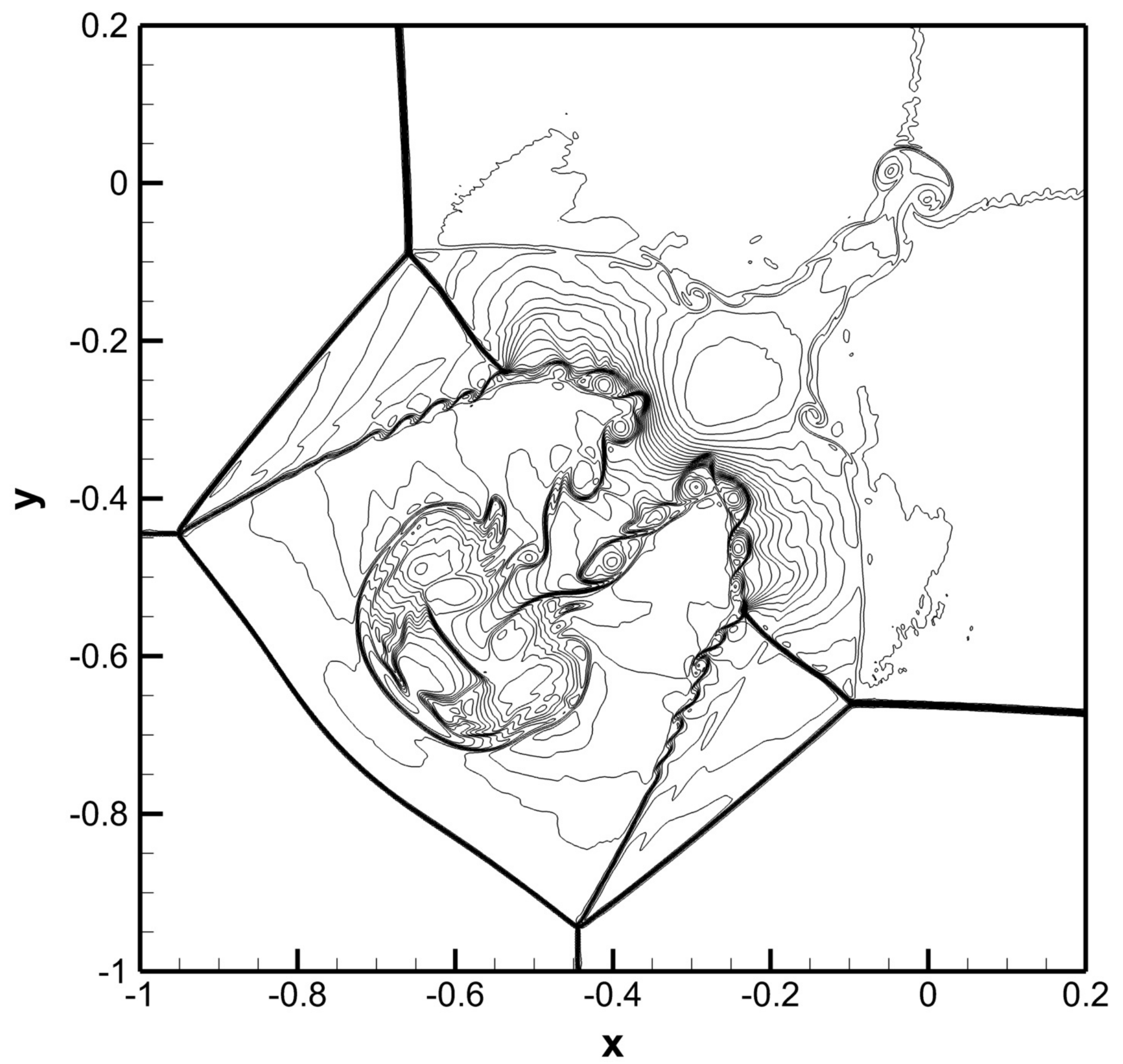




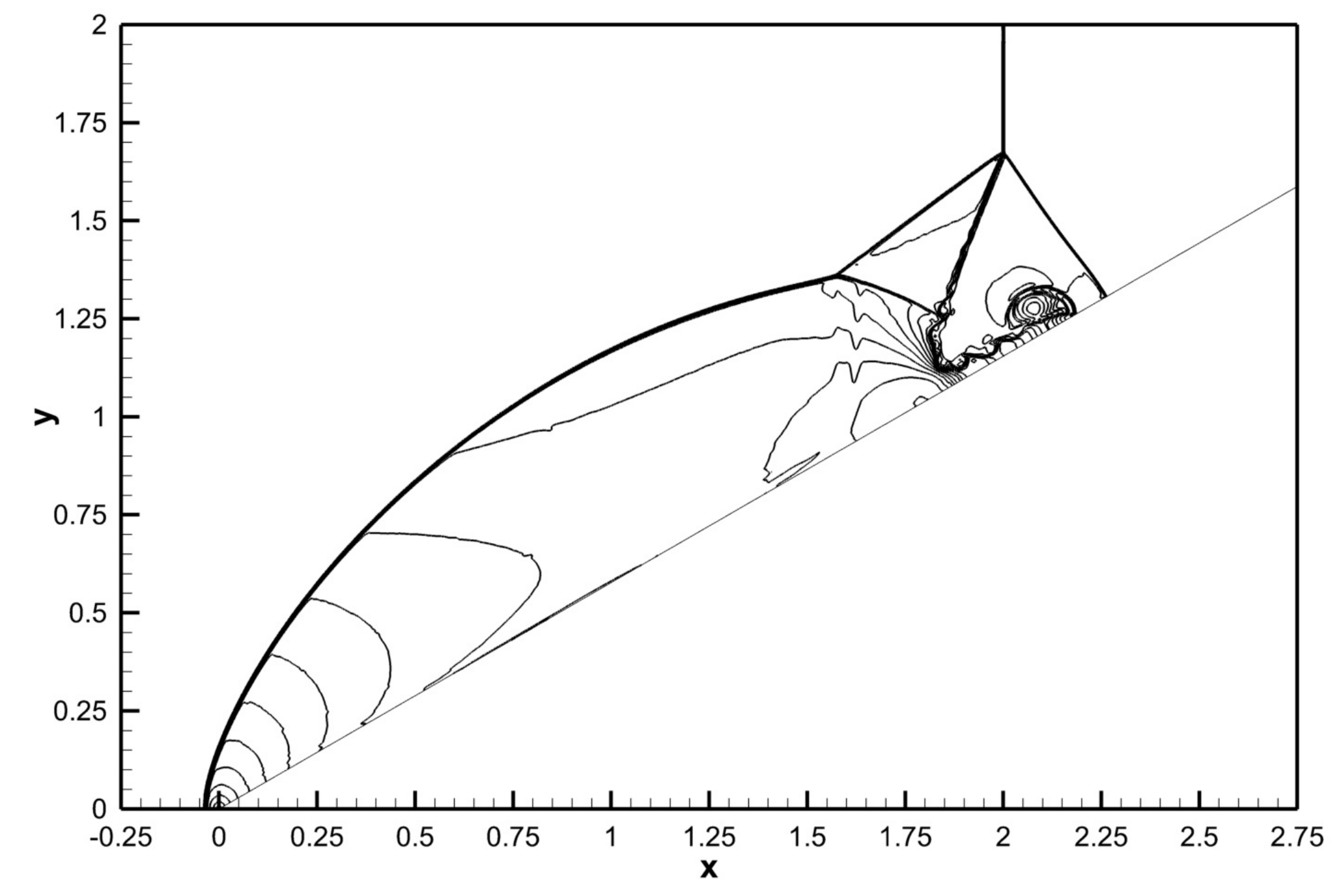

Fig9a 


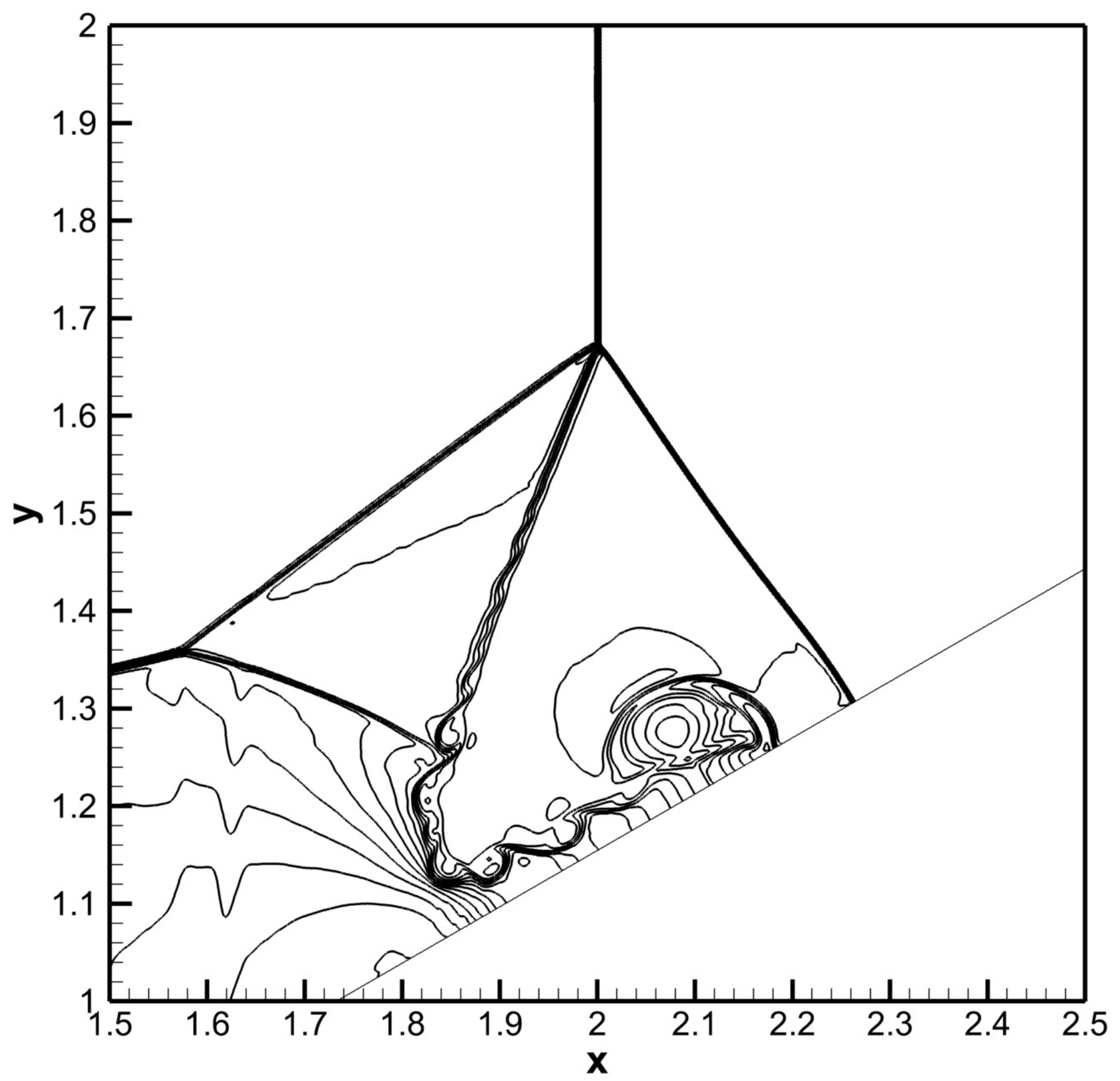




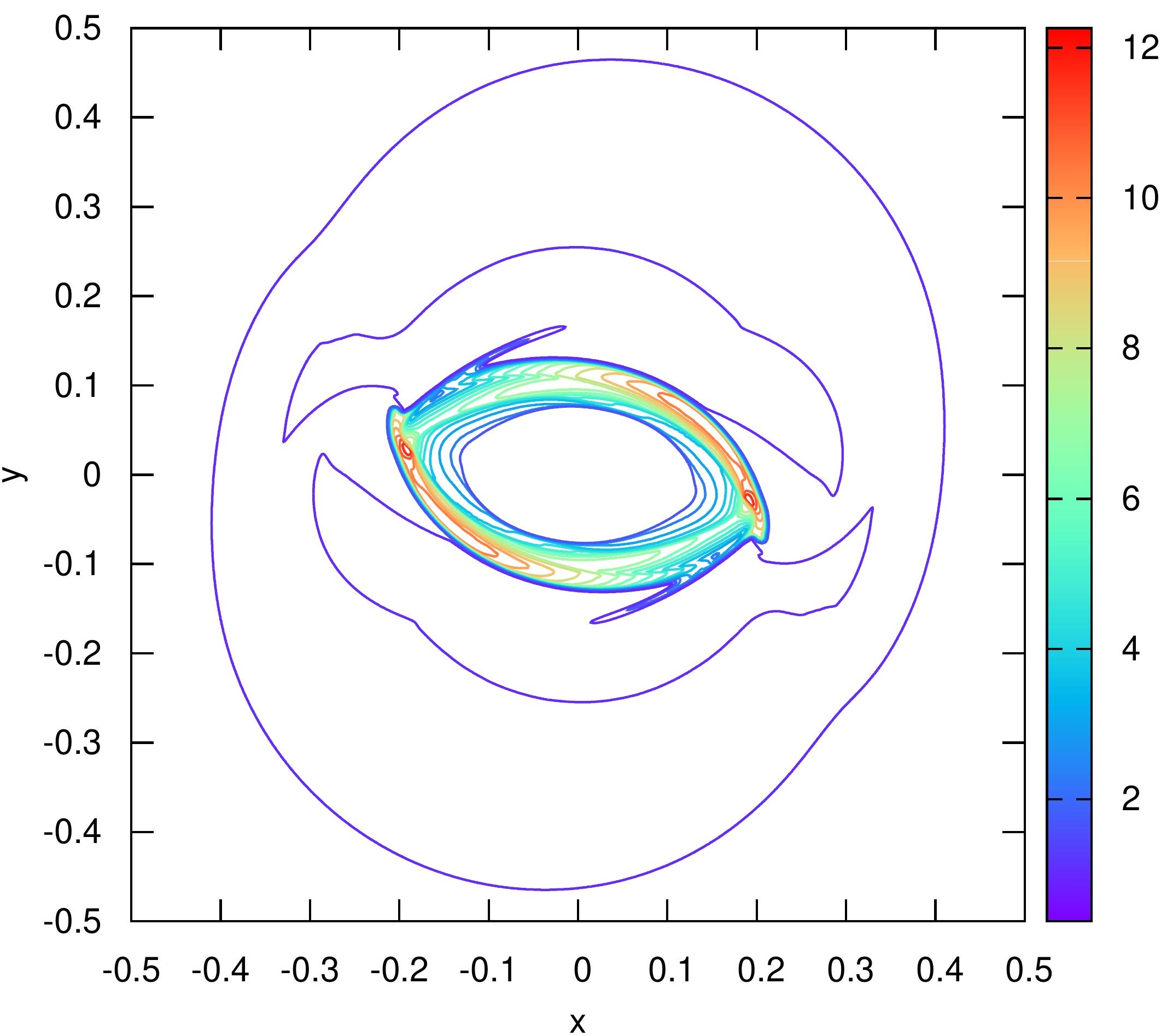




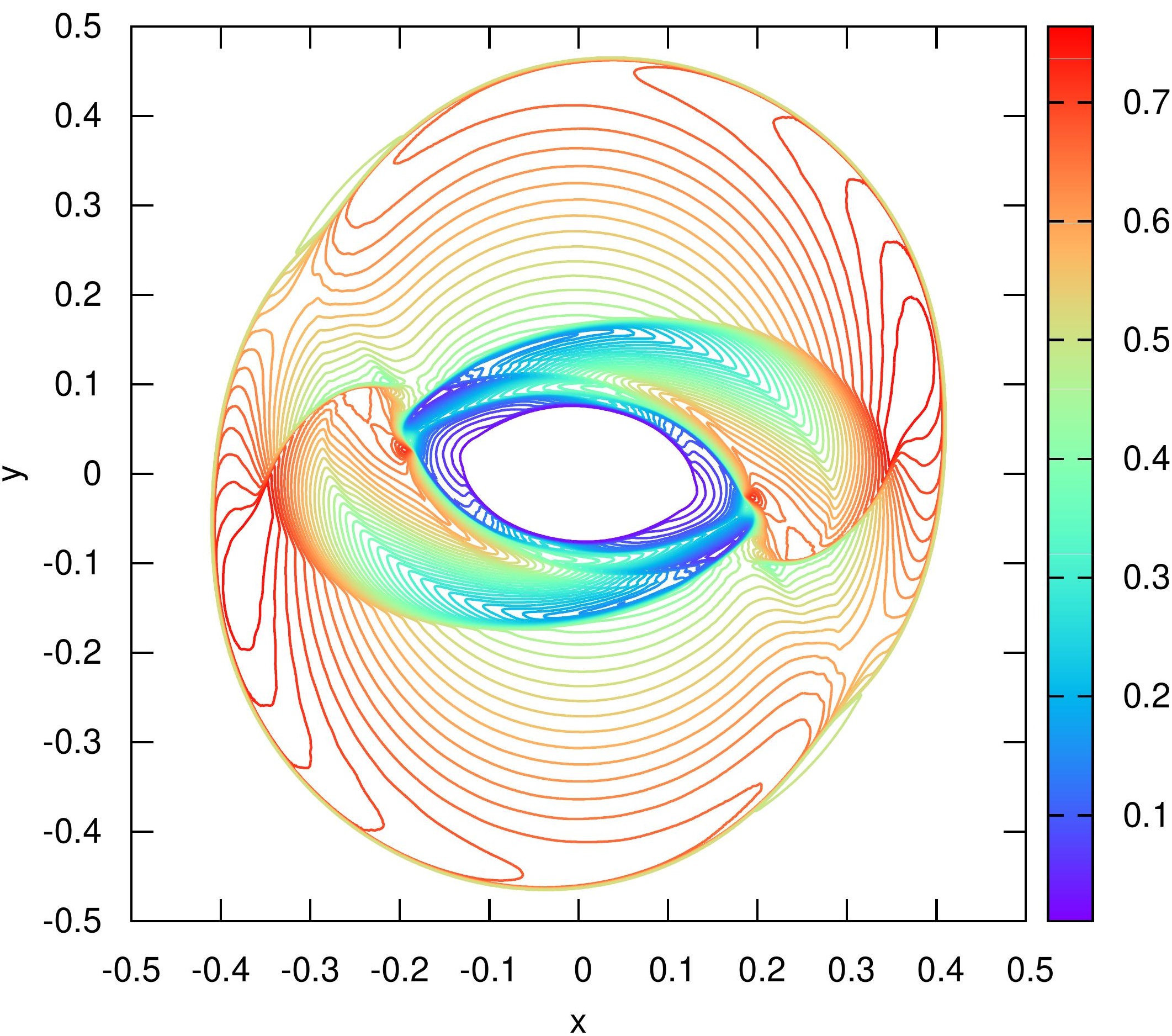




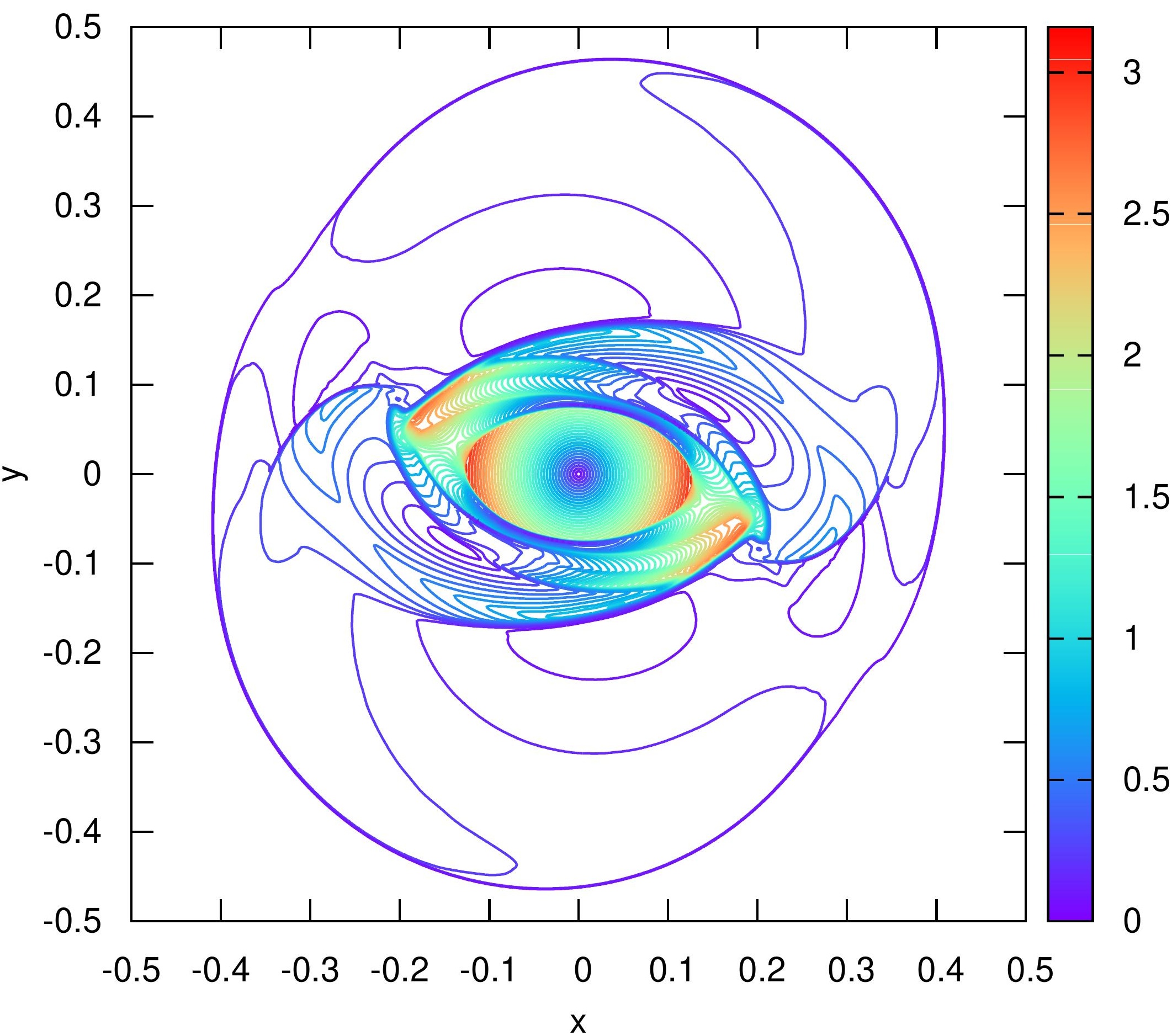




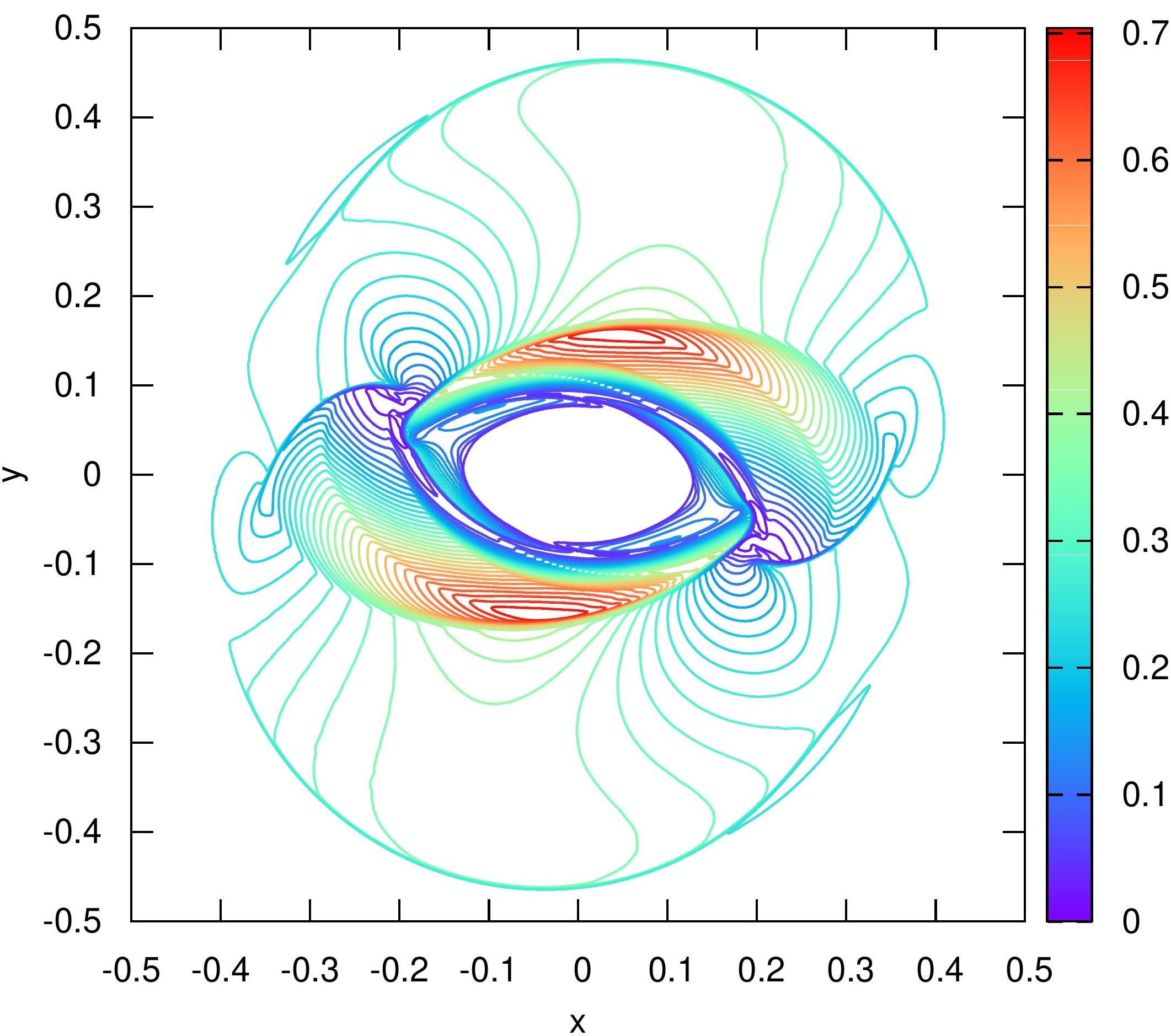




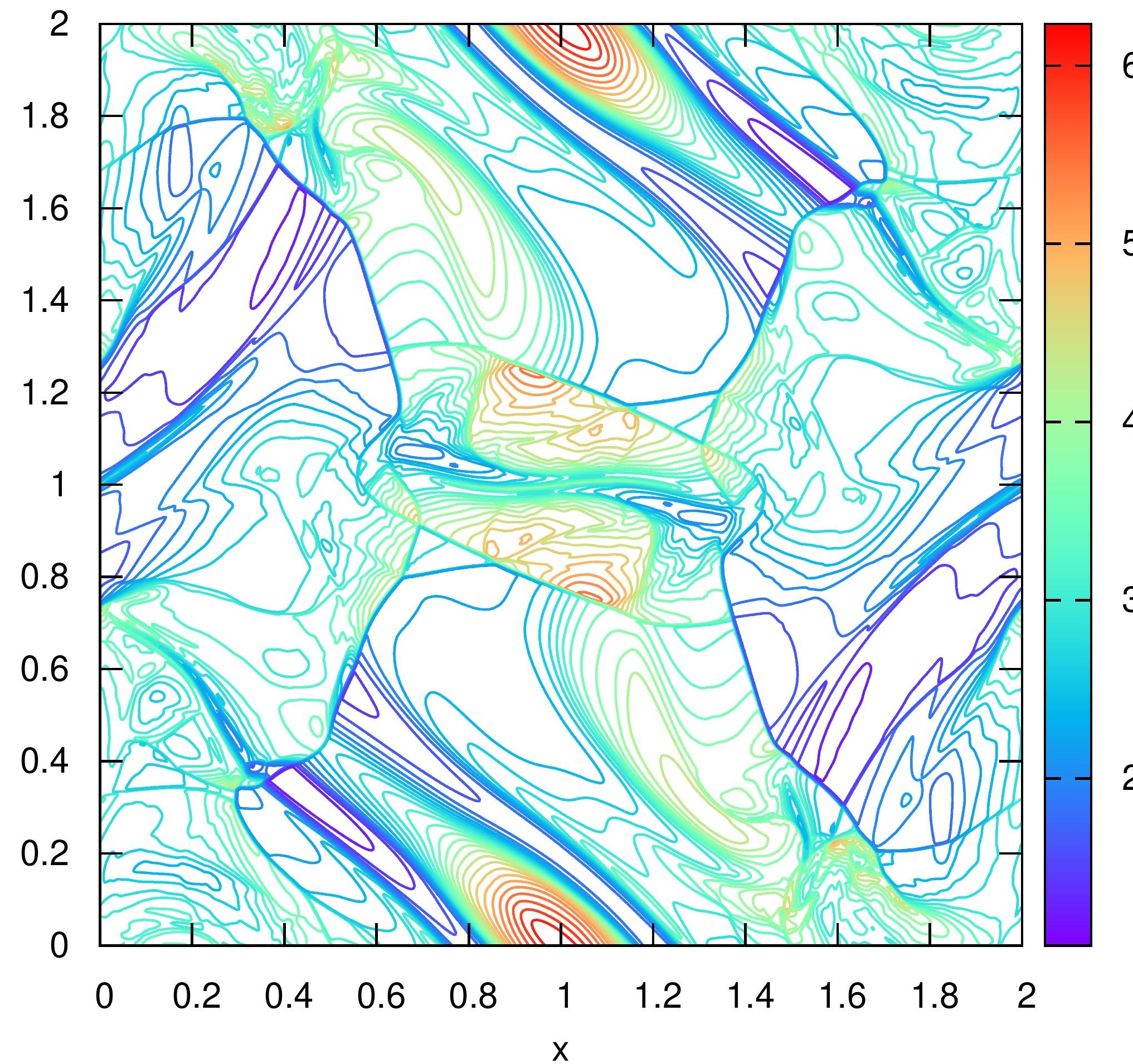




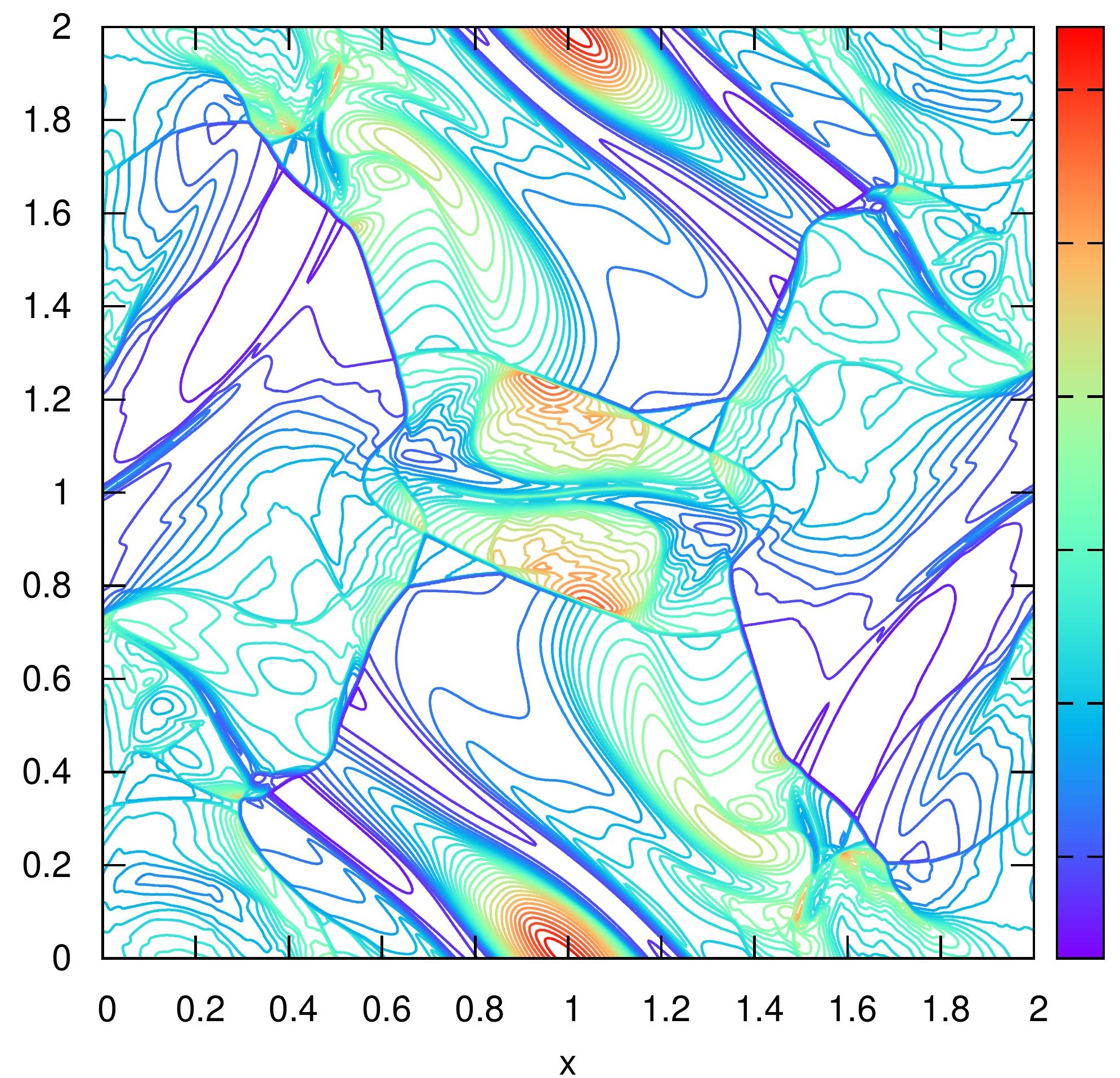

6
5
4

3
2
1

3 


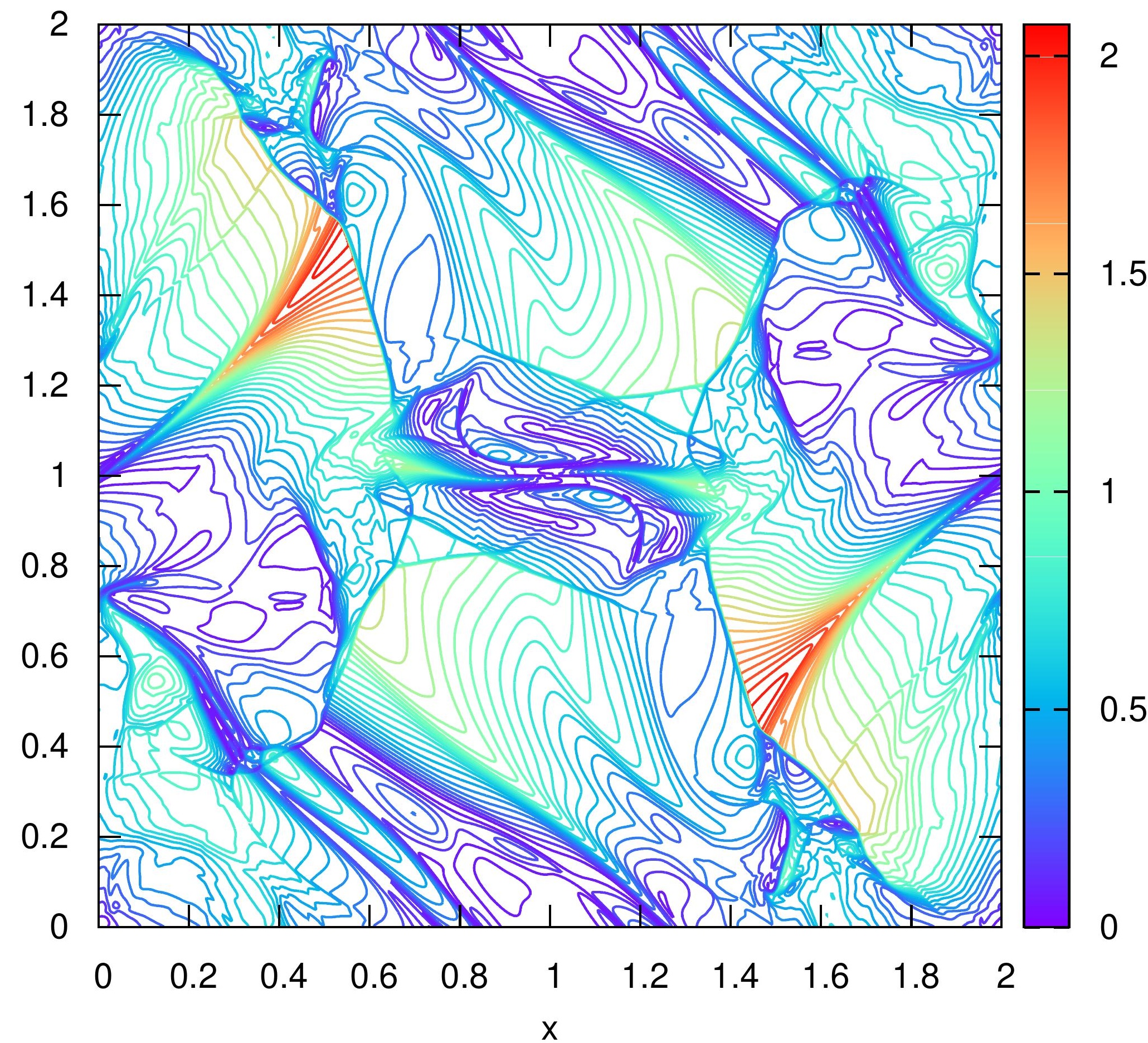




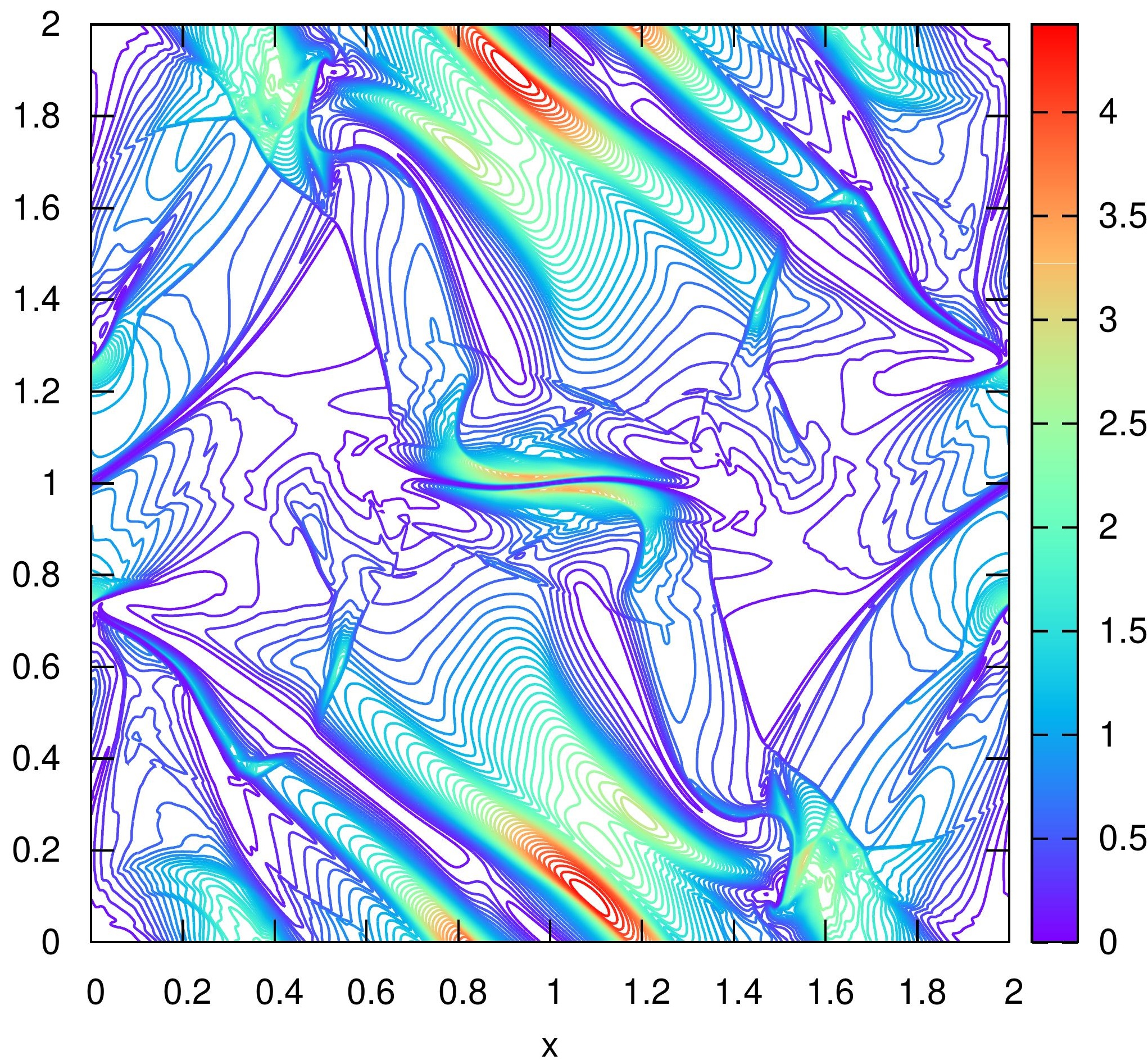




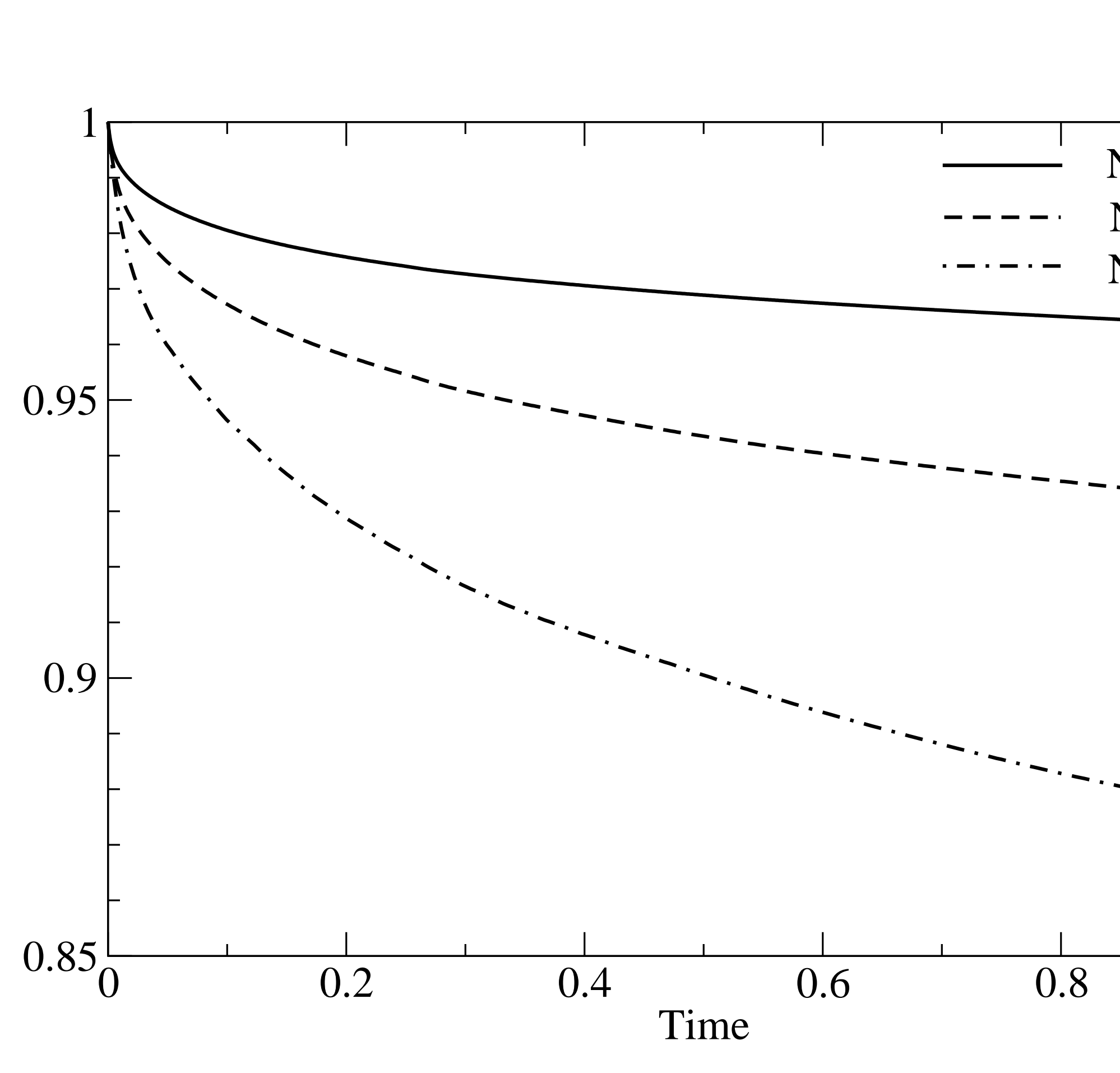

Time

Fig12a

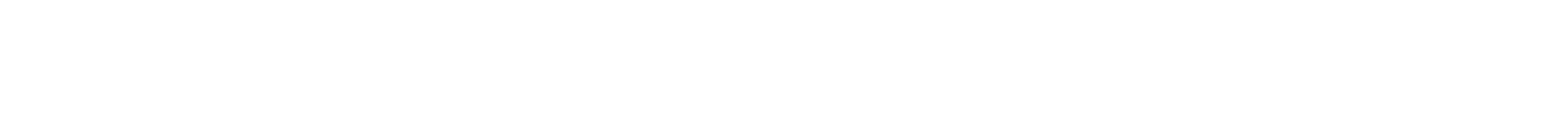




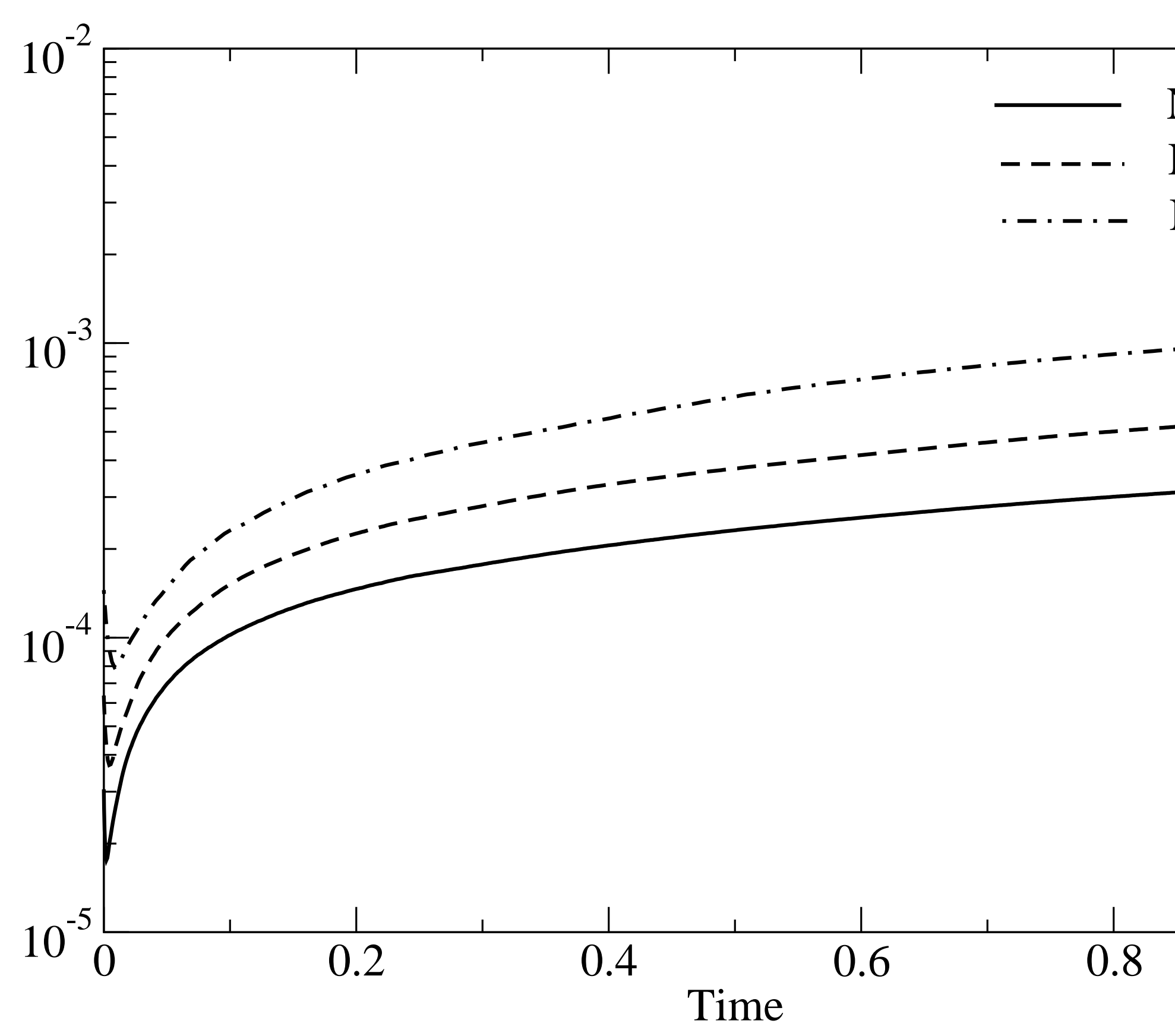

Time 


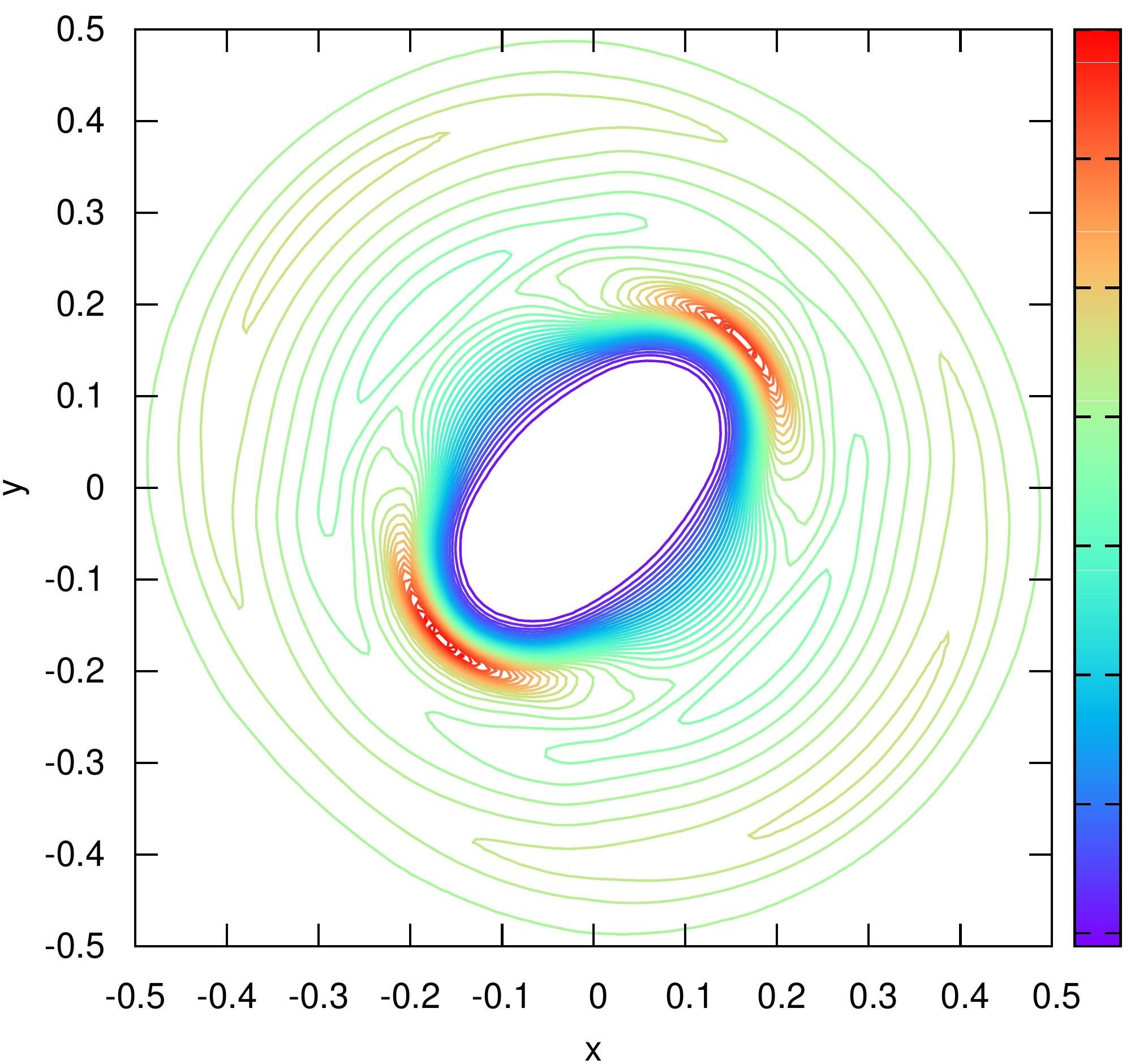

1.6

1.4

1.2

1

0.8

0.6

0.4

0.2

.

t 


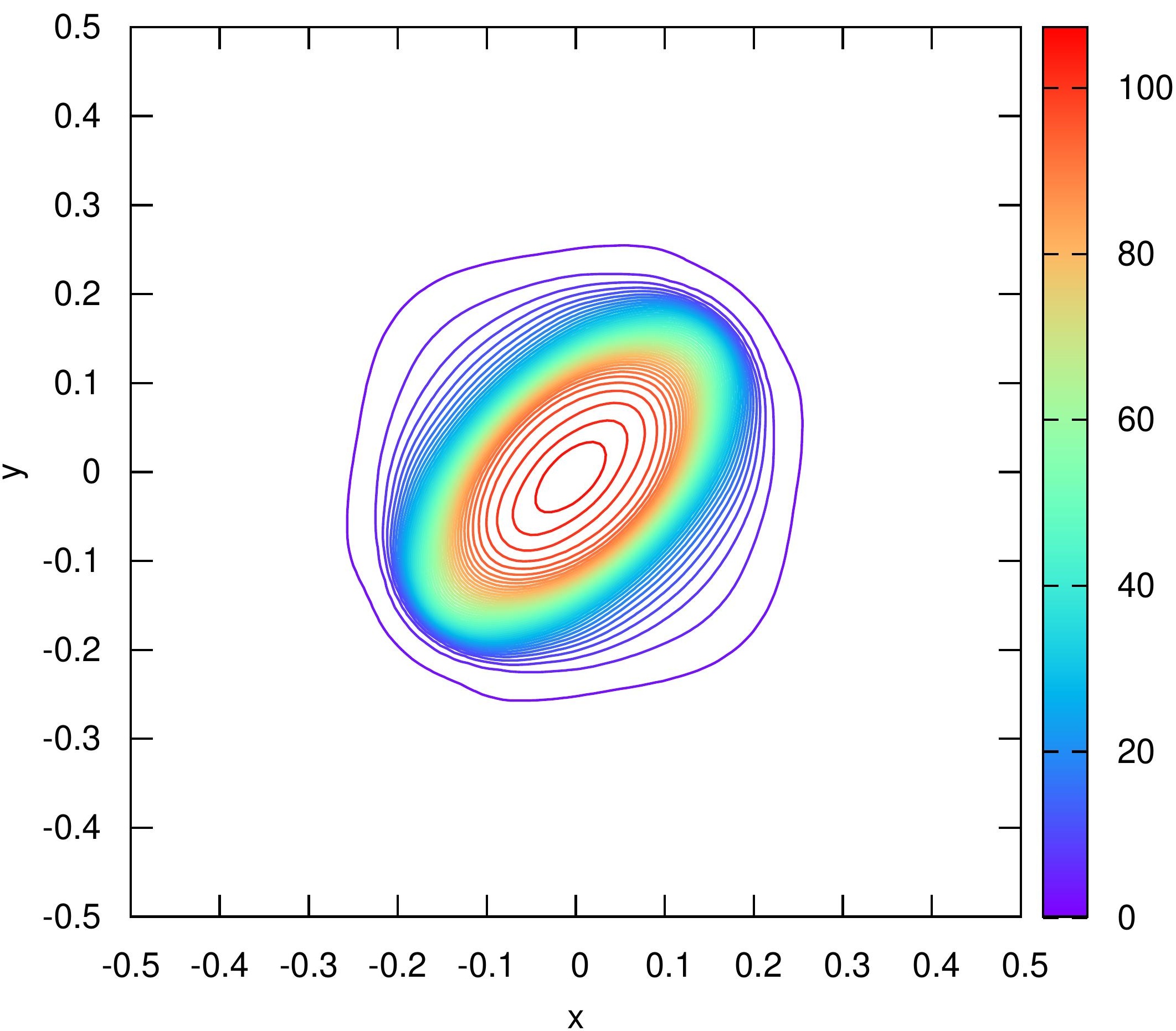




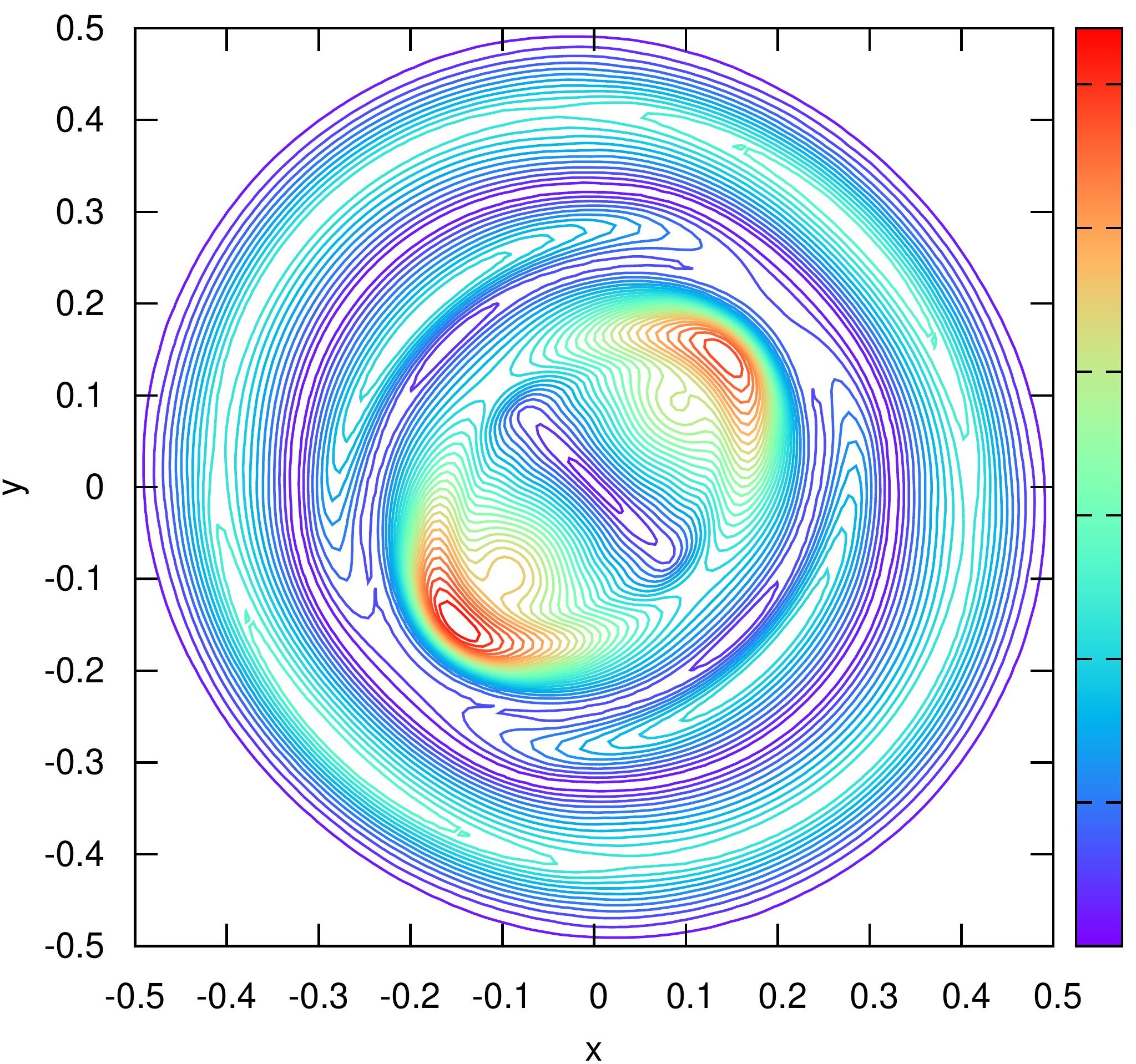

6

5

4

3

2

1

0 
